\title{
HOMOGENEOUS HYPERCOMPLEX STRUCTURES II - COSET SPACES OF COMPACT LIE GROUPS
}

\author{
GEORGE DIMITROV AND VASIL TSANOV
}

\begin{abstract}
We obtain a complete classification of hypercomplex manifolds, on which a compact group of automorphisms acts transitively. The description of the spaces as well as the proofs of our results use only the structure theory of reductive groups, in particular the notion of "stem" of a reduced root system, introduced in the first paper of this series.
\end{abstract}

\section{CONTEnts}

1. Introduction

2. The stem revisited

2.1. Notations

2.2. The stem

2.3. Decompositions

3. Hypercomplex pairs

3.1. Background and definition

3.2. Construction of a hypercomplex structure on $T_{o}(M)$

4. Necessary conditions

4.1. Introduction

4.2. $M$ as a coset space of a complex Lie group.

4.3. Construction of I, J

4.4. Fixing the Cartan subalgebra

4.5. Regularity of $\mathfrak{k}$ and $\mathfrak{p}$

4.6. The set $\Delta_{\mathfrak{p}}^{+}$

4.7. Decomposition of the Cartan subalgebra: $\mathfrak{h}_{u}, \mathfrak{h}_{\mathfrak{k}_{u}}, \mathfrak{h}_{\mathfrak{p}_{u}}, \mathfrak{n}_{\mathfrak{p}_{u}} \quad 23$

4.8. Preliminary lemmas about $\mathfrak{k}, \mathfrak{p}^{+}, \mathfrak{p}^{-}$

4.9. The integrability condition

4.10. Results for J. Determining of the subsets $\Delta_{\mathfrak{k}}^{+}, \Delta_{\mathfrak{p}}^{+} \quad 30$

4.11. The main theorem

5. Semisimple hypercomplex pairs

5.1. If $\mathfrak{g}$ is simple and $\operatorname{srank}(\mathfrak{g})=2 \operatorname{rank}(\mathfrak{g})$.

5.2. If $\mathfrak{g}=\mathfrak{s o}(2 p, \mathbb{C}), p=2 q+1, q \geq 2$

5.3. If $\mathfrak{g}=\mathfrak{e}_{6}$.

5.4. If $\mathfrak{g}=\operatorname{sl}(n+1, \mathbb{C}), n \geq 1$.

5.5. Semisimple hypercomplex pairs 
5.6. HC spaces $\quad 44$

References

\section{INTRODUCTION}

This paper is the second part in a series. In the first part 6] we introduced a remarkable subset 1 of a reduced root system, called the stem $\Gamma=\Gamma\left(\Delta^{+}\right)$. Using this we obtained a complete description of all left-invariant hypercomplex structures on a compact Lie group U.

Here we describe all left-invariant hypercomplex structures (for brevity we often write LIHCS further) on a coset space $M=\mathbf{U} / \mathbf{K}_{u}$, where $\mathbf{U}$ is a compact, connected Lie group and $\mathbf{K}_{u}$ is a closed subgroup. In particular we describe all simply-connected, compact, homogeneous, hypercomplex spaces. We have chosen to assume that $\mathbf{U}$ acts effectively on $M$ (we always may), with this restriction the underlying manifold of $\mathbf{U}$, does not in general admit a LIHCS, but factoring by an appropriate subgroup $\mathbf{K}_{u}$, we may get a coset space $M$, which carries a LIHCS.

Nevertheless, it is natural to try to obtain a homogeneous hypercomplex coset space starting with a group $\mathbf{U}$, which admits LIHCS and factoring by a subgroup $\mathbf{K}_{u}$, which is invariant under the given LIHCS. A careful reading of [6] and the present arguments should convince the reader that the idea works.

Following this idea in section 3 we define the notion of hypercomplex pair: a pair $\left(\mathfrak{u}, \mathfrak{k}_{\mathfrak{u}}\right)$ of compact Lie algebras $\mathfrak{u} \supset \mathfrak{k}_{u}$ satisfying certain conditions, essentially expressed in terms of the stems of the (reductive) complexifications $\mathfrak{g}, \mathfrak{k}$ of $\mathfrak{u}, \mathfrak{k}_{u}$ respectively.

Further we construct a LIHCS on any coset space $\mathbf{U} / \mathbf{K}_{u}$ provided that the pair of Lie algebras $\left(\mathfrak{u}, \mathfrak{k}_{\mathfrak{u}}\right)$ corresponding to $\mathbf{U}, \mathbf{K}_{u}$ is a hypercomplex pair and $\mathbf{K}_{u}$ is connected.

In Section 4 we prove that if $\mathbf{U} / \mathbf{K}_{u}$ admits a LIHCS then $\left(\mathfrak{u}, \mathfrak{k}_{\mathfrak{u}}\right)$ is a hypercomplex pair and the LIHCS has the form described in Section 3. For the sake of completeness, in the first part of this section we describe in detail and prove (some maybe known) structural results on the nonkaehler complex homogeneous spaces.

Actually in sections 3 , 4 we solve the invariant hypercomplex equations on a coset space $\mathbf{U} / \mathbf{K}_{u}$ :

$$
\begin{gathered}
I, J \in C_{i n v}^{\infty}\left(\mathbf{U} / \mathbf{K}_{u}, \operatorname{End}\left(T\left(\mathbf{U} / \mathbf{K}_{u}\right)\right)\right. \\
I \circ J=-J \circ I, \quad I^{2}=J^{2}=-1, \quad N_{J}=N_{I}=0,
\end{gathered}
$$

where $N_{I}, N_{J}$ are the Nijenhuis tensors of $I, J$ respectively. So, we find all LIHCS on $\mathbf{U} / \mathbf{K}_{u}$ and describe them on the language of the stem.

In section 5 we show that the hypercompex pairs $\left(\mathfrak{u}, \mathfrak{k}_{u}\right)$ with semi-simple $\mathfrak{u}$ are composed of $s u$-algebras. Then we show that any compact, simply connected homogeneous hypercomplex

\footnotetext{
${ }^{1}$ determined by a choice of a basis, that is up to the Weyl group action
} 
space is associated to a hypercompex pair $\left(\mathfrak{u}, \mathfrak{k}_{u}\right)$ with semi-simple $\mathfrak{u}$. Finally, using our description of semisimple hypercomplex pairs we give complete list of the HC spaces.

Thus we give, in terms of structure theory only, a complete classification of the homogeneous hypercomplex spaces with a transitive action of a compact group.

Less explicit results under additional constraints on the input data in the context of differential geometry have appeared in [2].

There is still a large class of compact homogeneous hypercomplex spaces unclassified, essentially those obtained from a hypercomplex structure on a nonabelian noncompact Lie group, by factorization with an uniform lattice.

\section{THE STEM REVISITED}

In this section we fix some notations used throughout the paper and recall the notion of stem, introduced in [6], and some of its properties.

2.1. Notations. By $\mathbf{U}$ and $\mathfrak{u}$ we shall denote a compact Lie group and its Lie algebra. We have a decomposition of $\mathfrak{u}$ as follows

$$
\mathfrak{u}=\mathfrak{c}_{u} \oplus \mathfrak{u}_{s}
$$

where $\mathfrak{c}_{u}$ is the center of $\mathfrak{u}$ and $\mathfrak{u}_{s}$ is a semisimple subalgebra.

We shall denote by $\mathfrak{g}$ the complexification of $\mathfrak{u}$ and by $\tau$ - the respective conjugation. The complexification of (11) will be denoted

$$
\mathfrak{g}=\mathfrak{c} \oplus \mathfrak{g}_{s}
$$

By $\mathfrak{h}, \Delta, \Delta^{+}, \Pi$ we shall denote respectively a $\tau$-invariant Cartan subalgebra of $\mathfrak{g}$, the root system of $\mathfrak{g}$ w. r. to $\mathfrak{h}$, set of positive roots, and a basis $\Pi$ of $\Delta$.

We use the following notations related to the the Cartan subalgebra $\mathfrak{h}$ :

$$
\mathfrak{h}=\mathfrak{c} \oplus \mathfrak{h}_{s}, \quad \text { where } \quad \mathfrak{h}_{s}=\mathfrak{h} \cap \mathfrak{g}_{s}, \quad \mathfrak{h}_{u}=\mathfrak{h} \cap \mathfrak{u} .
$$

For $\alpha \in \Delta$, we denote $\mathfrak{g}(\alpha)=\{X \in \mathfrak{g} \mid[H, X]=\alpha(H) X, \quad H \in \mathfrak{h}\}$.

Let $h_{\alpha} \in \mathfrak{h}_{s}$ be determined by $\operatorname{Kill}\left(H, h_{\alpha}\right)=\alpha(H)$ for $H \in \mathfrak{h}_{s}$, we denote $H_{\alpha}=$ $\left(2 / \alpha\left(h_{\alpha}\right)\right) h_{\alpha}$.

We also choose $E_{\alpha} \in \mathfrak{g}(\alpha)$ so that

$$
\tau\left(E_{\alpha}\right)=\tau_{s}\left(E_{\alpha}\right)=-E_{-\alpha}, \quad\left[E_{\alpha}, E_{\beta}\right]=N_{\alpha, \beta} E_{\alpha+\beta}, \quad\left[E_{\alpha}, E_{-\alpha}\right]=H_{\alpha} .
$$

and the structural constants $N_{\alpha, \beta}$ are integer.

Thus $\left\{E_{\alpha}, \mid \alpha \in \Delta\right\} \cup\left\{H_{\beta} \mid \beta \in \Pi\right\}$ is a Weyl - Chevalley basis of $\mathfrak{g}_{s}$. Having fixed a $\tau$-invariant Cartan subalgebra $\mathfrak{h}$ and a $\Delta^{+}$, we have the standard nilpotent and Borelian subalgebras, which will be denoted respectively:

$$
\mathfrak{n}^{+}=\bigoplus_{\alpha \in \Delta^{+}} \mathfrak{g}(\alpha), \quad \mathfrak{n}^{-}=\tau\left(\mathfrak{n}^{+}\right) ; \quad \mathfrak{b}^{+}=\mathfrak{n}^{+} \oplus \mathfrak{h}, \quad \mathfrak{b}^{-}=\tau\left(\mathfrak{b}^{+}\right)
$$


For $X, Y \in \mathfrak{g}$, we denote by $\langle X, Y\rangle$ an ad-invariant symmetric bilinear form such that its restriction to the compact real form $\mathfrak{u}$ is negative definite. We assume that $\langle.,$.$\rangle coincides$ with the Killing form on the semisimple part $\mathfrak{g}_{s}$.

2.2. The stem. In [6], for any $\zeta \in \Delta^{+}$we defined

$$
\Phi_{\zeta}^{+}=\left\{\beta \in \Delta^{+} \mid \zeta-\beta \in \Delta^{+}\right\}, \quad \Phi_{\zeta}=\Phi_{\zeta}^{+} \cup\left(-\Phi_{\zeta}^{+}\right) .
$$

We proved in [6], that there exists exactly one subset $\Gamma \subset \Delta^{+}$, such that

$$
\Delta^{+}=\Gamma \cup \bigcup_{\gamma \in \Gamma} \Phi_{\gamma}^{+} \quad \text { disjoint union }
$$

and we called this $\Gamma$ the stem of $\Delta^{+}$. By construction, the stem carries a certain uniquely determined partial order $\prec$ which is extremely important for the results (and proofs) in this paper, so we underline this fact by adopting the notation $(\Gamma, \prec)$ for the stem.2

The following properties of the stem $(\Gamma, \prec)$ will be used throughout the paper:

Proposition 2.1. Let $\gamma, \delta \in \Gamma$ and $\alpha \in \Phi_{\gamma}^{+} \cup\{\gamma\}, \beta \in \Phi_{\delta}^{+} \cup\{\delta\}$ then

$$
\begin{gathered}
\delta \succ \gamma \text { and } \alpha \pm \beta \in \Delta \Rightarrow \alpha \pm \beta \in \Phi_{\gamma}^{+} \\
\delta=\gamma \text { and } \alpha+\beta \in \Delta \Rightarrow \alpha+\beta=\gamma \\
\delta \prec \gamma \Rightarrow \alpha \pm \delta \notin \Delta, \quad \text { in particular } \alpha\left(H_{\delta}\right)=0 ; \\
\text { if not }(\gamma \preceq \delta \text { or } \gamma \succeq \delta) \text { then } \alpha \pm \beta \notin \Delta, \quad \text { in particular } \alpha\left(H_{\delta}\right)=0 ;
\end{gathered}
$$$$
\alpha-\gamma \in \Delta^{-}, \alpha+\gamma \notin \Delta, \quad \text { in particular } \alpha\left(H_{\gamma}\right)>0 \text {; }
$$$$
\text { there exists a maximal root } \widetilde{\gamma} \in \Gamma \text { s. t. } \gamma \succeq \widetilde{\gamma} \text {; }
$$

$$
\delta \prec \gamma \Rightarrow \quad \exists \beta_{1}, \beta_{2} \in \Phi_{\delta}^{+} \quad \alpha=\beta_{1}-\beta_{2} .
$$

Proof. First we recall (see [7], p. 456/457) that for any $\alpha, \beta \in \Delta$ we have $2 \frac{\langle\alpha, \beta\rangle}{\langle\beta, \beta\rangle}=\alpha\left(H_{\beta}\right)=$ $-p-q$, where $\{\alpha+n \beta, p \leq n \leq q\}$ is the $\beta$-series of $\alpha$.

Statements (5), (7), (99) follow from formulas (23), (21), (22) in Corollary 2.17 of [6], respectively. Now (6) follows from a) in Corrolary 2.11 of [6].

We go to the proof of (10). Let $\widetilde{\Delta}$ be the irreducible component of $\Delta$, s. t. $\gamma \in \widetilde{\Delta}$. Then $\widetilde{\Delta}^{+}=\widetilde{\Delta} \cap \Delta^{+}$is a system of positive roots in $\widetilde{\Delta}$. Let's take the highest root of $\left(\widetilde{\Delta}, \widetilde{\Delta}^{+}\right)$and denote it by $\widetilde{\gamma}$. Then $\widetilde{\gamma}$ is a maximal root of $\left(\Delta, \Delta^{+}\right)$. From the construction of the stem in Proposition 2.9, [6] we know that $\widetilde{\gamma} \in \Gamma$. From Proposition 2.15, [6] (and its proof) we know that $\Theta_{\widetilde{\gamma}}=\widetilde{\Delta}$. Thus we see that $\gamma \in \Theta_{\widetilde{\gamma}}$, which by definition (Definition 2.16 in [6]) means $\gamma \succeq \widetilde{\gamma}$

We go to the proof of (8) . Let $\Pi$ be the basis of $\Delta$ with positive roots $\Delta^{+}$. From Proposition 2.9, [6] we get a sequence $\Delta=\Delta_{1} \supset \Delta_{2} \supset \cdots \supset \Delta_{d}$ of closed root subsystems

\footnotetext{
${ }^{2}$ If $w$ is an automorphism of the root system $\Delta$, then $w(\Gamma)$ is the stem of $w\left(\Delta^{+}\right)$. We can recover $\Delta^{+}$ from the data $(\Gamma, \prec)$ but different choices of Weyl chambers may lead to the same set of roots $\Gamma$.
} 
with bases $\Pi_{k}=\Pi \cap \Delta_{k}$, corresponding sets of positive roots $\Delta_{k}^{+}=\Delta^{+} \cap \Delta_{k} \supset \Phi_{\gamma_{k}}^{+}$, and maximal roots $\gamma_{k}$ of $\Delta_{k}^{+}, \quad k=1, \ldots, d$. Furthermore the stem is $\Gamma=\left\{\gamma_{1}, \gamma_{2}, \ldots, \gamma_{d}\right\}$. Let $\gamma=\gamma_{i}, \delta=\gamma_{j}$. We can assume that $i<j$. Now $\gamma, \delta \in \Delta_{i}^{+}, \Phi_{\gamma}^{+}, \Phi_{\delta}^{+} \subset \Delta_{i}^{+}$. Let $\Delta^{\prime}, \Delta^{\prime \prime}$ be the irreducible components of $\Delta_{i}$, s. t. $\gamma \in \Delta^{\prime}, \delta \in \Delta^{\prime \prime}$. Since $\gamma$ is a maximal root of $\Delta_{i}$, from Proposition 2.15, 6] we see that $\Theta_{\gamma}=\Delta^{\prime}$ and since $\gamma$ and $\delta$ are incomparable then $\delta \notin \Delta^{\prime}$. Hence $\Delta^{\prime}$ and $\Delta^{\prime \prime}$ are different irreducible components of $\Delta_{i}$. Since $\Delta^{\prime}$ and $\Delta^{\prime \prime}$ are irreducible components of $\Delta_{i}, \gamma \in \Delta^{\prime}, \delta \in \Delta^{\prime \prime}$ and $\Phi_{\gamma}^{+}, \Phi_{\delta}^{+} \subset \Delta_{i}^{+}$one can easily observe that

$$
\Phi_{\gamma}^{+}=\left\{\zeta \in \Delta_{i}^{+} \mid \gamma-\zeta \in \Delta_{i}^{+}\right\} \subset \Delta^{\prime} \quad \Phi_{\delta}^{+}=\left\{\zeta \in \Delta_{i}^{+} \mid \delta-\zeta \in \Delta_{i}^{+}\right\} \subset \Delta^{\prime \prime} .
$$

Hence $\alpha \in \Delta^{\prime}$ and $\beta \in \Delta^{\prime \prime}$. Since $\Delta^{\prime}$ and $\Delta^{\prime \prime}$ are different irreducible components of $\Delta_{i}$ then $\alpha \pm \beta \notin \Delta_{i}$. Since $\Delta_{i}$ is a closed subsystem of $\Delta$, then $\alpha \pm \beta \notin \Delta$.

Obviously, for the proof of (11) it is sufficient to consider irreducible $\Delta$ with $\delta$ - the highest root of $\Delta^{+}$. Case by case through Section 3.3 of [6] one easily verifies (11).

Corollary 2.2. $\Gamma$ may be represented as a sequence $\left\{\gamma_{1}, \ldots, \gamma_{d}\right\}$, in which the first members are the maximal roots ( $i$. e. there is a number $1 \leq d^{\prime} \leq d$, such that $\gamma_{i}$ is a maximal root iff $\left.1 \leq i \leq d^{\prime}\right)$ and for any $\gamma_{i}, \gamma_{j} \in \Gamma$ we have $\gamma_{i} \prec \gamma_{j} \Rightarrow i<j$.

For any reductive Lie algebra $\mathfrak{g}$ we define a number $\operatorname{srank}(\mathfrak{g})$. If $\mathfrak{g}$ is not abelian we take any Cartan subalgebra $\mathfrak{h}$ with root system $\Delta$ and for any choice of a Weyl chamber we get a set of positive roots $\Delta^{+}$, hence we get the stem $(\Gamma, \prec)$ of $\Delta^{+}$. From the uniqueness of the stem ( [6], Theorem 2.12), we see that the number $\#(\Gamma)$ is a well defined characteristic of $\mathfrak{g}$.

Definition 2.3. If $\mathfrak{g}$ is abelian we set $\operatorname{srank}(\mathfrak{g})=0$. For a nonabelian reductive Lie algebra $\mathfrak{g}$ we denote: $\operatorname{srank}(\mathfrak{g})=2 \#(\Gamma)$.

2.3. Decompositions. At the end of this section we recall some useful decompositions related to the stem. Next we define important subspaces of $\mathfrak{h}$ and $\mathfrak{h}_{u}$.

Definition 2.4. We denote

$$
\mathfrak{w}=\operatorname{span}_{\mathbb{C}}\left\{H_{\gamma} \mid \gamma \in \Gamma\right\}, \quad \mathfrak{o}=\bigcap_{\gamma \in \Gamma} \mathfrak{k} \mathfrak{e r}(\gamma) ; \quad \mathfrak{w}_{u}=\mathfrak{w} \cap \mathfrak{u}, \quad \mathfrak{o}_{u}=\mathfrak{o} \cap \mathfrak{u} .
$$

Obviously we have orthogonal decompositions:

$$
\mathfrak{h}=\mathfrak{w} \oplus \mathfrak{o}, \quad \mathfrak{h}_{u}=\mathfrak{w}_{u} \oplus \mathfrak{o}_{u}
$$

Definition 2.5. Let $\rho=\left\{\rho_{\gamma} \mid \gamma \in \Gamma\right\}$ be a family of complex numbers on the unit circle. For each $\gamma \in \Delta^{+}$we denote:

$$
\begin{gathered}
W_{\gamma}=\frac{\mathrm{i}}{2} H_{\gamma}, \quad X_{\gamma}(\rho)=\frac{1}{2}\left(\rho_{\gamma} E_{\gamma}-\overline{\rho_{\gamma}} E_{-\gamma}\right), \quad Y_{\gamma}(\rho)=\frac{\mathrm{i}}{2}\left(\rho_{\gamma} E_{\gamma}+\overline{\rho_{\gamma}} E_{-\gamma}\right), \\
\operatorname{sl}_{\gamma}(2)=\operatorname{span}_{\mathbb{C}}\left\{E_{\gamma}, E_{-\gamma}, H_{\gamma}\right\}, \quad \operatorname{su}_{\gamma}(2)=\operatorname{span}_{\mathbb{R}}\left\{X_{\gamma}, Y_{\gamma}, W_{\gamma}\right\} .
\end{gathered}
$$


Definition 2.6. As in [6], for each $\gamma \in \Gamma$, we shall denote:

$$
\mathcal{V}_{\gamma}^{+}=\sum_{\alpha \in \Phi_{\gamma}^{+}} \mathfrak{g}(\alpha), \quad \mathcal{V}_{\gamma}^{-}=\sum_{\alpha \in \Phi_{\gamma}^{-}} \mathfrak{g}(\alpha), \quad \mathcal{V}_{\gamma}=\mathcal{V}_{\gamma}^{+} \oplus \mathcal{V}_{\gamma}^{-}, \quad \mathcal{V}_{\gamma}^{u}=\mathfrak{u} \cap \mathcal{V}_{\gamma} .
$$

We have again orthogonal decompositions:

$$
\mathfrak{g}=\mathfrak{o} \oplus \bigoplus_{\gamma \in \Gamma}\left(s l_{\gamma}(2) \oplus \mathcal{V}_{\gamma}\right), \quad \mathfrak{u}=\mathfrak{o}_{u} \oplus \bigoplus_{\gamma \in \Gamma}\left(s u_{\gamma}(2) \oplus \mathcal{V}_{\gamma}^{u}\right)
$$

\section{HyPERCOMPLEX PAIRS}

3.1. Background and definition. Let $I$ be a left-invariant complex structure on $\mathbf{U}$, and let us denote also by $I$ its restriction to the tangent space at the unit element, which we identify with $\mathfrak{u}$. We still denote by $I$ the $\mathbb{C}$ - linear extention of $I$ to $\mathfrak{g}$. Then it is well known (see e.g. [14]), that we may find a $\tau$-invariant Cartan subalgebra $\mathfrak{h}$, a basis $\Pi$ of the root system $\Delta$ w.r. to $\mathfrak{h}$, and a decomposition

$$
\mathfrak{h}=\mathfrak{h}^{+} \oplus \mathfrak{h}^{-}, \quad \tau\left(\mathfrak{h}^{+}\right)=\mathfrak{h}^{-},
$$

so that the regular subalgebras:

$$
\mathfrak{g}^{+}=\mathfrak{h}^{+} \oplus \mathfrak{n}^{+}, \quad \mathfrak{g}^{-}=\mathfrak{h}^{-} \oplus \mathfrak{n}^{-}
$$

are respectively the i, - i eigenspaces of $I$.

In [6] we have shown that a left-invariant complex structure $I$ on $\mathbf{U}$, presented in the above manner, admits a matching complex structure $J$ (i.e. $I J=-J I$ ) if and only if

$$
\mathfrak{z}=I \mathfrak{w} \subset \mathfrak{o}, \quad \operatorname{dim}(\mathbf{U}) \in \mathbf{4} \mathbb{Z} .
$$

We introduce notations for the entities related to the complex structure $I$ :

Definition 3.1. We denote

$$
\begin{aligned}
& Z_{\gamma}=I W_{\gamma}, \quad P_{\gamma}=W_{\gamma}-\mathrm{i} Z_{\gamma}, \quad Q_{\gamma}=W_{\gamma}+\mathrm{i} Z_{\gamma} . \\
& u_{\gamma}(2)=s u_{\gamma}(2) \oplus \mathbb{R} Z_{\gamma}, \quad g l_{\gamma}(2)=s l_{\gamma}(2) \oplus \mathbb{C} Z_{\gamma} .
\end{aligned}
$$

In [6] we have shown that any invariant hypercomplex structure $I, J$ on $\mathbf{U}$ splits the Lie algebra $\mathfrak{g}$ in the following form

$$
\mathfrak{g}=\mathfrak{j} \oplus \bigoplus_{\gamma \in \Gamma}\left(g l_{\gamma}(2) \oplus \mathcal{V}_{\gamma}\right), \quad \mathfrak{u}=\mathfrak{j}_{u} \oplus \bigoplus_{\gamma \in \Gamma}\left(u_{\gamma}(2) \oplus \mathcal{V}_{\gamma}^{u}\right)
$$

where $\mathfrak{o}_{u}=\mathfrak{j}_{u} \oplus I\left(\mathfrak{w}_{u}\right), \quad \operatorname{dim}_{\mathbb{R}}\left(\mathfrak{j}_{u}\right) \in 4 \mathbb{Z}$. In terms of this decomposition the hypercomplex structure $(I, J)$ is defined for $A \in \mathcal{V}_{\gamma}^{u}$ by:

$$
I A=2\left[W_{\gamma}, A\right], \quad J A=-2\left[Y_{\gamma}, A\right],
$$

for each $\gamma \in \Gamma$ it is determined on $u_{\gamma}(2)$ by:

$$
I X_{\gamma}=Y_{\gamma}, \quad I W_{\gamma}=Z_{\gamma} ; \quad J X_{\gamma}=W_{\gamma}, \quad J Z_{\gamma}=Y_{\gamma}
$$


and the hypercomplex structure on $\mathfrak{j}$ is defined by identifying it with $\mathbb{H}^{k}$. It is obvious that the decomposition subspaces $\mathfrak{j}_{u}, \mathfrak{u}_{\gamma}(2), \mathcal{V}_{\gamma}^{u}$ are $(I, J)$-invariant.

We shall obtain a left-invariant hypercomplex structure on a coset space $\mathbf{U} / \mathbf{K}_{u}$, when $\mathfrak{k}$ is a partial sum (of vector subspaces)

$$
\mathfrak{k}=\mathfrak{j}_{\mathfrak{k}} \oplus \bigoplus_{\gamma \in \Gamma_{\mathfrak{k}}}\left(g l_{\gamma}(2) \oplus \mathcal{V}_{\gamma}\right)
$$

where the subset $\Gamma_{\mathfrak{k}} \subset \Gamma$ and the subspace $\mathfrak{j}_{\mathfrak{k}} \subset \mathfrak{j}$ are properly chosen, so that the above $\mathfrak{k}$ is a subalgebra3.

Taking a complement $\mathfrak{j}_{\mathfrak{p}} \subset \mathfrak{j}$ so that $\mathfrak{p}=\mathfrak{j}_{\mathfrak{p}} \oplus \mathfrak{j}_{\mathfrak{k}}$ and denoting $\Gamma_{\mathfrak{p}}=\Gamma \backslash \Gamma_{\mathfrak{k}}$ we obtain a complement (see the decomposition (15)) of $\mathfrak{k}$ in $\mathfrak{g}$ :

$$
\mathfrak{p}=\mathfrak{j}_{\mathfrak{p}} \oplus \sum_{\gamma \in \Gamma \backslash \Gamma_{\mathfrak{k}}}\left(\mathcal{V}_{\gamma} \oplus g l_{\gamma}(2)\right)
$$

Then we use the same formulas as in (16), (17) to define a hypercomplex structure on $\mathfrak{p}$. In this section we carry out this program in detail. Further we prove that all LIHCS on our kind of coset spaces are obtained in this way 4 . We start by studying subalgebras $\mathfrak{k}$ as above. We start by studying subalgebras $\mathfrak{k}$ as above.

Obviously, for $\mathfrak{k}$ we have $\mathfrak{o} \cap \mathfrak{k}=\mathfrak{j}_{\mathfrak{k}}+\operatorname{span}_{\mathbb{C}}\left\{I\left(W_{\gamma}\right)\right\}_{\gamma \in \Gamma_{\mathfrak{k}}}$ and we can represent $\mathfrak{k}$ as follows

$$
\mathfrak{k}=(\mathfrak{o} \cap \mathfrak{k})+\sum_{\gamma \in \Gamma_{\mathfrak{k}}}\left(s l_{\gamma}(2)+\mathcal{V}_{\gamma}\right) .
$$

From property (11) of the stem $\Gamma$, it follows easily that if $\gamma, \delta \in \Gamma$, then

$$
\left(\gamma \in \Gamma_{\mathfrak{k}} \text { and } \delta \succ \gamma\right) \Rightarrow \delta \in \Gamma_{\mathfrak{k}} .
$$

Furthermore, we have

$$
\begin{aligned}
\mathfrak{h} \cap \mathfrak{k} & =\mathfrak{j}_{\mathfrak{k}} \oplus \operatorname{span}_{\mathbb{C}}\left\{W_{\gamma}\right\}_{\gamma \in \Gamma_{\mathfrak{k}}} \oplus \operatorname{span}_{\mathbb{C}}\left\{I\left(W_{\gamma}\right)\right\}_{\gamma \in \Gamma_{\mathfrak{k}}} ; \\
\mathfrak{o} & =\mathfrak{j}_{\mathfrak{k}} \oplus \mathfrak{j}_{\mathfrak{p}} \oplus \operatorname{span}_{\mathbb{C}}\left\{I\left(W_{\gamma}\right)\right\}_{\gamma \in \Gamma}
\end{aligned}
$$

therefore

$$
\operatorname{rank}(\mathfrak{g})+\operatorname{srank}(\mathfrak{k})-\operatorname{rank}(\mathfrak{k})-\operatorname{srank}(\mathfrak{g})=\operatorname{dim}\left(\mathfrak{j}_{\mathfrak{p}}\right) \geq 0 .
$$

It is convenient to introduce

Definition 3.2. A subset $\Gamma_{\mathfrak{k}} \subset \Gamma$, which satisfies (19), will be called a substem of $(\Gamma, \prec)$.

We have

\footnotetext{
${ }^{3}$ such that the subgroup generated by $\mathfrak{k} \cap \mathfrak{u}$ is closed

${ }^{4}$ It is easy to see from the examples in [6] and Section [5] of this paper, that even if we discard the "toral" part $\mathfrak{j}$, the action of $\mathbf{U}$ will not be effective in general
} 
Lemma 3.3. Let $\Gamma_{\mathfrak{k}} \subset \Gamma$ be a substem of $(\Gamma, \prec)$ and let $\Delta_{\mathfrak{k}}^{+}=\bigcup_{\gamma \in \Gamma_{\mathfrak{k}}} \Phi_{\gamma}^{+} \cup\{\gamma\}, \Delta_{\mathfrak{k}}=$ $\bigcup_{\gamma \in \Gamma_{\mathfrak{k}}} \Phi_{\gamma} \cup\{\gamma,-\gamma\}$. Then there exists a subset $\mathcal{M} \subset \Gamma$, which consists of pairwise incomparable with respect to $\prec$ elements and such that

$$
\Delta_{\mathfrak{k}}=\bigcup_{\gamma \in \mathcal{M}} \Theta_{\gamma}, \quad \Delta_{\mathfrak{k}}^{+}=\bigcup_{\gamma \in \mathcal{M}} \Theta_{\gamma}^{+} \quad \text { disjoint unions }
$$

(for the definition of $\left\{\Theta_{\gamma}\right\}_{\gamma \in \Gamma}$ see Proposition 2.15 in [6]).

The stem of $\Delta_{\mathfrak{k}}^{+}$is $\Gamma_{\mathfrak{k}}$.

Proof. We denote by $\mathcal{M}$ the set of all minimal elements in $\Gamma_{\mathfrak{k}}$ (with respect to the ordering $\prec$ ). Let us take any element $\alpha \in \Delta_{\mathfrak{k}}^{+}$then by $\Delta_{\mathfrak{k}}^{+}=\bigcup_{\gamma \in \Gamma_{\mathfrak{k}}} \Phi_{\gamma}^{+} \cup\{\gamma\}$ it follows that $\alpha \in \Phi_{\gamma}^{+} \cup\{\gamma\}$ for some $\gamma \in \Gamma_{\mathfrak{k}}$. Since $\Gamma_{\mathfrak{k}}$ is finite then there exists a minimal element $\delta \in \mathcal{M}$ such that $\delta \preceq \gamma$ and therefore $\gamma \in \Theta_{\delta}^{+}$and $\Phi_{\gamma}^{+} \subset \Theta_{\delta}^{+}$. So we see that $\Delta_{\mathfrak{k}}^{+} \subset \bigcup_{\gamma \in \mathcal{M}} \Theta_{\gamma}^{+}$.

Let us take any $\gamma \in \mathcal{M}$. We recall that the stem of $\Theta_{\gamma}^{+}$is $\Gamma \cap \Theta_{\gamma}$ and

$$
\Theta_{\gamma}^{+}=\bigcup_{\delta \in \Gamma \cap \Theta_{\gamma}} \Phi_{\delta}^{+} \cup\{\delta\}
$$

Since all the elements in $\Gamma \cap \Theta_{\gamma}$ are greater than $\gamma \in \Gamma_{\mathfrak{k}}$ and by (19) it follows that $\Gamma \cap \Theta_{\gamma} \subset \Gamma_{\mathfrak{k}}$. Therefore $($ see (22) $)$ it follows that $\Delta_{\mathfrak{k}}^{+} \supset \Theta_{\gamma}^{+}$, hence $\Delta_{\mathfrak{k}}^{+} \supset \bigcup_{\gamma \in \mathcal{M}} \Theta_{\gamma}^{+}$.

Thus far, we proved (21). Obviously that for any pair of different elements $\gamma, \delta \in \mathcal{M}$ neither $\gamma \preceq \delta$ nor $\gamma \succeq \delta$. Hence in (21) we have disjoint unions.

For $\gamma \in \mathcal{M}$ the stem of $\left(\Theta_{\gamma}, \Theta_{\gamma}^{+}\right)$is $\Gamma \cap \Theta_{\gamma}$, then the stem of $\left(\Delta_{\mathfrak{k}}, \Delta_{\mathfrak{k}}^{+}\right)$is $\cup_{\gamma \in \mathcal{M}} \Gamma \cap \Theta_{\gamma}=$ $\Gamma \cap \Delta_{\mathfrak{k}}=\Gamma_{\mathfrak{k}}$ and the corollary is completely proved.

For a subalgebra $\mathfrak{k}$, which satisfies (18), we give a definition

Definition 3.4. Let $(\Gamma, \prec)$ be a stem of a reductive algebra $\mathfrak{g}$. Let $\Gamma_{\mathfrak{k}} \subset \Gamma$ be a substem of $(\Gamma, \prec)$. A subalgebra $\mathfrak{k} \subset \mathfrak{g}$ of the type (18) will be called a $\Gamma_{\mathfrak{k}}$-stemmed subalgebra of $\mathfrak{g}$.

We come to the most important notion in this section

Definition 3.5. Let $\mathfrak{u} \supset \mathfrak{k}_{\mathfrak{u}}$ be a pair of compact Lie algebras. Let $\mathfrak{g} \supset \mathfrak{k}$ be the complexifications of $\left(\mathfrak{u}, \mathfrak{k}_{u}\right)$.

We call $\left(\mathfrak{u}, \mathfrak{k}_{u}\right)$ a hypercomplex pair if the following conditions are satisfied:

- $\operatorname{dim}(\mathfrak{u})-\operatorname{dim}\left(\mathfrak{k}_{u}\right)>0$ is divisible by four,

- There exist a Cartan subalgebra $\mathfrak{h}_{u} \subset \mathfrak{u}$ with complexification $\mathfrak{h} \subset \mathfrak{g}$, set of positive roots $\Delta^{+} \subset \Delta w$. r. to $\mathfrak{h}$, and stem $\Gamma \subset \Delta^{+}$, such that $\mathfrak{k}$ is a $\Gamma_{\mathfrak{k}}$-stemmed subalgebra of $\mathfrak{g}$ for some substem $\Gamma_{\mathfrak{k}} \subset \Gamma$.

- $\operatorname{rank}(\mathfrak{g})+\operatorname{srank}(\mathfrak{k}) \geq \operatorname{rank}(\mathfrak{k})+\operatorname{srank}(\mathfrak{g})$.

Let $\left(\mathfrak{u}, \mathfrak{k}_{u}\right)$ be a hypercomplex pair. A pair of groups $\left(\mathbf{U}, \mathbf{K}_{u}\right)$ is said to be associated to the hypercomplex pair $\left(\mathfrak{u}, \mathfrak{k}_{u}\right)$, if $\mathbf{U}$ is a compact, connected Lie group, $\mathfrak{u}$ is its Lie algebra, $\mathbf{K}_{u} \subset \mathbf{U}$ is a closed subgroup with Lie algebra $\mathfrak{k}_{u}$. 
Throughout the rest of this section $\left(\mathfrak{u}, \mathfrak{k}_{u}\right)$ is a hypercomplex pair and $(\mathfrak{g}, \mathfrak{k})$ is its complexification.

We assume also that we are given a pair of groups $\left(\mathbf{U}, \mathbf{K}_{u}\right)$, associated with $\left(\mathfrak{u}, \mathfrak{k}_{u}\right)$, where $\mathbf{K}_{u}$ is a connected subgroup of $\mathbf{U}$ 5. In this section we obtain a hypercomplex structure on any coset space $M=\mathbf{U} / \mathbf{K}_{u}$ of this type. We shall denote by $o \in M$ the coset $\mathbf{K}_{u} \in \mathbf{U} / \mathbf{K}_{u}$. Let $\mathfrak{h}$ and $\Gamma$ be as in Definition $[3.5$, applied to $(\mathfrak{g}, \mathfrak{k})$, so we have

$$
\begin{gathered}
\mathfrak{k}=\mathfrak{o}_{\mathfrak{k}} \oplus \bigoplus_{\gamma \in \Gamma_{\mathfrak{k}}}\left(\operatorname{sl}_{\gamma}(2) \oplus \mathcal{V}_{\gamma}\right), \quad \text { where } \quad \mathfrak{o}_{\mathfrak{k}}=\mathfrak{k} \cap \mathfrak{o} \\
\operatorname{rank}(\mathfrak{g})+\operatorname{srank}(\mathfrak{k})-\operatorname{rank}(\mathfrak{k})-\operatorname{srank}(\mathfrak{g}) \geq 0,
\end{gathered}
$$

where $\Gamma_{\mathfrak{k}}$ is a substem of $\Gamma$.

\subsection{Construction of a hypercomplex structure on $T_{o}(M)$.}

3.2.1. The subspace $\mathfrak{p}_{u} \subset \mathfrak{u}$. Let $\mathfrak{p}_{u}$ be the orthogonal complement of $\mathfrak{k}_{u}$ with respect to $\langle$, and let $\mathfrak{p}$ be the complexification of $\mathfrak{p}_{u}$. We shall denote

$$
\begin{gathered}
\mathfrak{h}_{\mathfrak{p}_{u}}=\mathfrak{h} \cap \mathfrak{p}_{u} \quad \mathfrak{h}_{\mathfrak{p}}=\mathfrak{h} \cap \mathfrak{p} \quad \mathfrak{h}_{\mathfrak{k}_{u}}=\mathfrak{h} \cap \mathfrak{k}_{u} \quad \mathfrak{h}_{\mathfrak{k}}=\mathfrak{h} \cap \mathfrak{k} \\
\mathfrak{o}_{\mathfrak{p}_{u}}=\mathfrak{o} \cap \mathfrak{p}_{u} \quad \mathfrak{o}_{\mathfrak{p}}=\mathfrak{o} \cap \mathfrak{p} \quad \mathfrak{o}_{\mathfrak{k}_{u}}=\mathfrak{o} \cap \mathfrak{k}_{u} .
\end{gathered}
$$

In order to describe $\mathfrak{p}$ in terms of the stem, it is convenient to introduce the following notatins

$$
\begin{gathered}
\Gamma_{\mathfrak{p}}=\Gamma \backslash \Gamma_{\mathfrak{k}} \quad \mathfrak{w}_{\mathfrak{p}}=\operatorname{span}_{\mathbb{R}}\left\{W_{\gamma} \mid \gamma \in \Gamma_{\mathfrak{p}}\right\} \quad \mathfrak{w}_{\mathfrak{k}}=\operatorname{span}_{\mathbb{R}}\left\{W_{\gamma} \mid \gamma \in \Gamma_{\mathfrak{k}}\right\} \\
\Delta_{\mathfrak{p}}^{+}=\bigcup_{\gamma \in \Gamma_{\mathfrak{p}}} \Phi_{\gamma}^{+} \cup\{\gamma\} \quad \Delta_{\mathfrak{p}}=\Delta_{\mathfrak{p}}^{+} \cup\left(-\Delta_{\mathfrak{p}}^{+}\right) \quad \Delta_{\mathfrak{k}}^{+}=\bigcup_{\gamma \in \Gamma_{\mathfrak{k}}} \Phi_{\gamma}^{+} \cup\{\gamma\} \quad \Delta_{\mathfrak{k}}=\Delta_{\mathfrak{k}}^{+} \cup\left(-\Delta_{\mathfrak{k}}^{+}\right) .
\end{gathered}
$$

From (23) we see that $\mathfrak{k}=\mathfrak{h}_{\mathfrak{k}}+\sum_{\alpha \in \Delta_{\mathfrak{k}}} \mathfrak{g}(\alpha)$ and $\mathfrak{g}=\mathfrak{k} \oplus \mathfrak{p}$. Hence we get

Lemma 3.6. The subspace $\mathfrak{p}$ may be decomposed as follows:

$$
\mathfrak{p}=\mathfrak{h}_{\mathfrak{p}} \oplus \bigoplus_{\alpha \in \Delta_{\mathfrak{p}}} \mathfrak{g}(\alpha)
$$

We have $\mathfrak{h}=\mathfrak{h}_{\mathfrak{k}} \oplus \mathfrak{h}_{\mathfrak{p}}$.

From (19) and $\Gamma_{\mathfrak{k}} \cap \Gamma_{\mathfrak{p}}=\emptyset$ it follows that if $\gamma \in \Gamma_{\mathfrak{p}}, \quad \delta \in \Gamma_{\mathfrak{k}}$, then either $\gamma \prec \delta$ or $\gamma, \delta$ are not comparable, so using Proposition 2.1, we have

$$
\gamma \in \Gamma_{\mathfrak{p}}, \beta \in \Delta_{\mathfrak{k}}, \alpha \in \Phi_{\gamma}, \alpha+\beta \in \Delta \Rightarrow \alpha+\beta \in \Phi_{\gamma}, \gamma \pm \beta \notin \Delta
$$

Lemma 3.7. $\mathfrak{w}_{\mathfrak{p}} \subset \mathfrak{p}_{\mathfrak{u}}$.

\footnotetext{
${ }^{5}$ So, by assumption, for the Lie algebra $\mathfrak{k}_{u}$ we have that the subgroup of $\mathbf{U}$ generated by $\mathfrak{k}_{u}$ is a closed subgroup, in addition to the properties listed in definition 3.5 .
} 
Proof. Let $\gamma \in \Gamma_{\mathfrak{p}}$. We have to prove that for all $X \in \mathfrak{k}$ we have $\left\langle W_{\gamma}, X\right\rangle=0$. To that end we recall (23) and the properties:

a) For each $\alpha \in \Delta$ the subspaces $\mathfrak{g}(\alpha)$ and $\mathfrak{h}$ are orthogonal with respect to $\langle$,$\rangle .$

b) $W_{\gamma}$ is orthogonal to $\mathfrak{o}$ with respect to $\langle$,$\rangle .$

c) For each $\delta \in \Gamma_{\mathfrak{k}} \gamma \pm \delta \notin \Delta$, in particular $\left\langle W_{\gamma}, W_{\delta}\right\rangle=0$.

Corollary 3.8. The following decompositions $\mathfrak{h}_{\mathfrak{p}}=\mathfrak{o}_{\mathfrak{p}} \oplus \mathfrak{w}_{\mathfrak{p}}^{\mathbb{C}}, \mathfrak{o}=\mathfrak{o}_{\mathfrak{k}} \oplus \mathfrak{o}_{\mathfrak{p}}$ are valid. In particular $\operatorname{dim}(\mathfrak{o})=\operatorname{dim}\left(\mathfrak{o}_{\mathfrak{k}}\right)+\operatorname{dim}\left(\mathfrak{o}_{\mathfrak{p}}\right)$. Furthermore, $\operatorname{dim}(\mathfrak{h})=\operatorname{dim}(\mathfrak{o})+\#(\Gamma), \operatorname{dim}\left(\mathfrak{h}_{\mathfrak{k}}\right)=$ $\operatorname{dim}\left(\mathfrak{o}_{\mathfrak{k}}\right)+\#\left(\Gamma_{\mathfrak{k}}\right)$.

Proof. Since for $\alpha \in \Delta$ and $H \in \mathfrak{h}$ we have $\alpha(\tau(H))=-\overline{\alpha(H)}$, then $\tau(\mathfrak{o})=\mathfrak{o}$, hence

$$
\mathfrak{o}=\mathfrak{o}_{u} \oplus \mathfrak{i} \mathfrak{o}_{u} \quad \mathfrak{o}_{\mathfrak{k}}=\mathfrak{o}_{\mathfrak{k}_{u}} \oplus \mathrm{i} \mathfrak{o}_{\mathfrak{k}_{u}} \quad \mathfrak{o}_{\mathfrak{p}}=\mathfrak{o}_{\mathfrak{p}_{u}} \oplus \mathfrak{i} \mathfrak{o}_{\mathfrak{p}_{u}}
$$

The orthogonal complement of $\operatorname{span}_{\mathbb{R}}\left\{W_{\gamma} \mid \gamma \in \Gamma\right\}$ in $\mathfrak{h}_{u}$ is $\mathfrak{o}_{u}$, hence $\operatorname{span}_{\mathbb{R}}\left\{W_{\gamma} \mid \gamma \in \Gamma\right\} \oplus \mathfrak{o}_{u}=$ $\mathfrak{h}_{u}$ and therefore $\operatorname{dim}\left(\mathfrak{h}_{u}\right)=\operatorname{dim}\left(\mathfrak{o}_{u}\right)+\#(\Gamma)$ and $\operatorname{dim}_{\mathbb{C}}(\mathfrak{h})=\operatorname{dim}_{\mathbb{C}}(\mathfrak{o})+\#(\Gamma)$. In Lemma 3.7 we proved that $W_{\gamma} \in \mathfrak{p}_{u}$ for $\gamma \in \Gamma_{\mathfrak{p}}$, besides by (23) we have $W_{\gamma} \in \mathfrak{k}_{u}$ for $\gamma \in \Gamma_{\mathfrak{k}}$. Since $\mathfrak{p}_{u}$ is orthogonal to $\mathfrak{k}_{u}$, in particular $\mathfrak{p}_{u}$ is orthogonal to $\mathfrak{h}_{\mathfrak{k}_{u}}$ as well(we recall that $\mathfrak{h}_{\mathfrak{k}_{u}}=\mathfrak{k}_{u} \cap \mathfrak{h}_{u}$ ), hence $\mathfrak{o}_{\mathfrak{k}_{u}}=\mathfrak{o} \cap \mathfrak{k}_{u}=\mathfrak{o} \cap \mathfrak{h}_{\mathfrak{k}_{u}}$ is the orthogonal complement of $\operatorname{span}\left\{W_{\gamma} \mid \gamma \in \Gamma_{\mathfrak{k}}\right\}$ in $\mathfrak{h}_{\mathfrak{k}_{u}}$, which implies $\mathfrak{w}_{\mathfrak{k}} \oplus \mathfrak{o}_{\mathfrak{k}_{u}}=\mathfrak{h}_{\mathfrak{k}_{u}}$, hence $\operatorname{dim}\left(\mathfrak{h}_{\mathfrak{k}}\right)=\operatorname{dim}\left(\mathfrak{o}_{\mathfrak{k}}\right)+\#\left(\Gamma_{\mathfrak{k}}\right)$. Besides $\mathfrak{o}_{\mathfrak{p}_{u}}=\mathfrak{o} \cap \mathfrak{p}_{u}=\mathfrak{o} \cap \mathfrak{h}_{\mathfrak{p}_{u}}$ is the orthogonal complement of $\operatorname{span}\left\{W_{\gamma} \mid \gamma \in \Gamma_{\mathfrak{p}}\right\}$ in $\mathfrak{h}_{\mathfrak{p}_{u}}$. Thus far, we showed that

$$
\begin{gathered}
\operatorname{span}_{\mathbb{R}}\left\{W_{\gamma} \mid \gamma \in \Gamma\right\} \oplus \mathfrak{o}_{u}=\mathfrak{h}_{u}, \\
\mathfrak{w}_{\mathfrak{k}} \oplus \mathfrak{o}_{\mathfrak{k}_{u}}=\mathfrak{h}_{\mathfrak{k}_{u}}, \quad \mathfrak{w}_{\mathfrak{p}} \oplus \mathfrak{o}_{\mathfrak{p}_{u}}=\mathfrak{h}_{\mathfrak{p}_{u}} .
\end{gathered}
$$

These equations together with $\mathfrak{h}_{u}=\mathfrak{h}_{\mathfrak{k}_{u}} \oplus \mathfrak{h}_{\mathfrak{p}_{u}}$ imply $\mathfrak{o}_{u}=\mathfrak{o}_{\mathfrak{k}_{u}} \oplus \mathfrak{o}_{\mathfrak{p}_{u}}$. Therefore $\mathfrak{o}=\mathfrak{o}_{\mathfrak{k}} \oplus \mathfrak{o}_{\mathfrak{p}}$.

Lemma 3.9. The following integers are equal to each other:

a) $\operatorname{rank}(\mathfrak{g})+\operatorname{srank}(\mathfrak{k})-\operatorname{rank}(\mathfrak{k})-\operatorname{srank}(\mathfrak{g})$

b) $\operatorname{dim}\left(\mathfrak{h}_{\mathfrak{p}}\right)-2 \#\left(\Gamma_{\mathfrak{p}}\right)$

c) $\operatorname{dim}\left(\mathfrak{o}_{\mathfrak{p}}\right)-\#\left(\Gamma_{\mathfrak{p}}\right)$.

Proof. By Corollary 3.8 we have $\operatorname{dim}(\mathfrak{o})=\operatorname{dim}\left(\mathfrak{o}_{\mathfrak{k}}\right)+\operatorname{dim}\left(\mathfrak{o}_{\mathfrak{p}}\right), \operatorname{besides} \operatorname{dim}(\mathfrak{h})=\operatorname{dim}(\mathfrak{o})+$ $\#(\Gamma), \operatorname{dim}(\mathfrak{h} \cap \mathfrak{k})=\operatorname{dim}\left(\mathfrak{o}_{\mathfrak{k}}\right)+\#\left(\Gamma_{\mathfrak{k}}\right)$. In the calculations that follow we take into account these equalities

$$
\begin{gathered}
\operatorname{dim}\left(\mathfrak{o}_{\mathfrak{p}}\right)-\#\left(\Gamma_{\mathfrak{p}}\right)=\operatorname{dim}(\mathfrak{o})-\operatorname{dim}\left(\mathfrak{o}_{\mathfrak{k}}\right)-\#\left(\Gamma_{\mathfrak{p}}\right)=\operatorname{dim}(\mathfrak{h})-\#(\Gamma) \\
-\left(\operatorname{dim}(\mathfrak{h} \cap \mathfrak{k})-\#\left(\Gamma_{\mathfrak{k}}\right)\right)-\left(\#(\Gamma)-\#\left(\Gamma_{\mathfrak{k}}\right)\right)=\operatorname{rank}(\mathfrak{g})-\operatorname{rank}(\mathfrak{k})-2 \#(\Gamma)+2 \#\left(\Gamma_{\mathfrak{k}}\right) \\
=\operatorname{rank}(\mathfrak{g})-\operatorname{rank}(\mathfrak{k})-\operatorname{srank}(\mathfrak{g})+\operatorname{srank}(\mathfrak{k})
\end{gathered}
$$

and the equality between $a$ ) and $c$ ) is proved. The equality between a) and b) follows from

$$
\begin{gathered}
\operatorname{dim}(\mathfrak{h} \cap \mathfrak{p})-2 \#\left(\Gamma_{\mathfrak{p}}\right)=\operatorname{rank}(\mathfrak{g})-\operatorname{rank}(\mathfrak{k})-2\left(\#(\Gamma)-\#\left(\Gamma_{\mathfrak{k}}\right)\right) \\
=\operatorname{rank}(\mathfrak{g})-\operatorname{rank}(\mathfrak{k})+\operatorname{srank}(\mathfrak{k})-\operatorname{srank}(\mathfrak{g}) .
\end{gathered}
$$


Since on one hand $\mathfrak{h}_{\mathfrak{p}} \cap \mathfrak{u}$ and $\mathfrak{h}_{\mathfrak{k}} \cap \mathfrak{u}$ are orthogonal to each other and on the other hand $H_{\gamma} \in \mathfrak{h}_{\mathfrak{p}}$ for $\gamma \in \Gamma_{\mathfrak{p}}$ (see Lemma 3.7), $H_{\alpha} \in \mathfrak{h}_{\mathfrak{k}}$ for $\alpha \in \Delta_{\mathfrak{k}}$ then one can easily verify that for any choice of $\gamma \in \Gamma_{\mathfrak{p}}, \alpha \in \Delta_{\mathfrak{k}}$ we have

$$
\gamma_{\mid \mathfrak{h}_{\mathfrak{k}}}=0 ; \quad \quad \alpha_{\mid \mathfrak{h}_{\mathfrak{p}}}=0 .
$$

We recall that $\operatorname{rank}(\mathfrak{g})+\operatorname{srank}(\mathfrak{k})-\operatorname{rank}(\mathfrak{k})-\operatorname{srank}(\mathfrak{g}) \geq 0$, hence, by Lemma $[3.9$ we may write

$$
\operatorname{dim}\left(\mathfrak{h}_{\mathfrak{p}}\right) \geq 2 \#\left(\Gamma_{\mathfrak{p}}\right) \quad \operatorname{dim}\left(\mathfrak{o}_{\mathfrak{p}}\right) \geq \#\left(\Gamma_{\mathfrak{p}}\right) .
$$

Let us give some comments on the dimension of $\mathfrak{o}_{\mathfrak{p}}$. First of all by (30) we have $\operatorname{dim}\left(\mathfrak{o}_{\mathfrak{p}}\right) \geq$ $\#\left(\Gamma_{\mathfrak{p}}\right)$, therefore $\operatorname{dim}_{\mathbb{R}}\left(\mathfrak{o}_{\mathfrak{p}_{u}}\right) \geq \#\left(\Gamma_{\mathfrak{p}}\right)$. By (26) we see that $\#\left(\Delta_{\mathfrak{p}}^{+}\right)+\#\left(\Gamma_{\mathfrak{p}}\right)$ is even (for any $\gamma \in \Gamma$ the set $\Phi_{\gamma}^{+}$contains of even number of elements). From (27) it follows that $\operatorname{dim}\left(\mathfrak{o}_{\mathfrak{p}}\right)-\#\left(\Gamma_{\mathfrak{p}}\right)=\operatorname{dim}(\mathfrak{p})-2 \#\left(\Gamma_{\mathfrak{p}}\right)-2 \#\left(\Delta_{\mathfrak{p}}^{+}\right)=\operatorname{dim}(\mathfrak{p})-2\left(\#\left(\Gamma_{\mathfrak{p}}\right)+\#\left(\Delta_{\mathfrak{p}}^{+}\right)\right)$. Since $\operatorname{dim}(\mathfrak{p})$ is divisible by four as well as $2\left(\#\left(\Gamma_{\mathfrak{p}}\right)+\#\left(\Delta_{\mathfrak{p}}^{+}\right)\right)$, then $\operatorname{dim}\left(\mathfrak{o}_{\mathfrak{p}}\right)-\#\left(\Gamma_{\mathfrak{p}}\right)$ is divisible by four. Therefore $\operatorname{dim}\left(\mathfrak{o}_{\mathfrak{p}_{u}}\right)-\#\left(\Gamma_{\mathfrak{p}}\right)$ is divisible by four and we can decompose $\mathfrak{o}_{\mathfrak{p}_{u}}$ as follows

$$
\mathfrak{o}_{\mathfrak{p}_{u}}=\mathfrak{j}_{\mathfrak{p}_{u}} \oplus \mathfrak{z}_{\mathfrak{p}} \quad \quad \operatorname{dim}_{\mathbb{R}}\left(\mathfrak{z}_{\mathfrak{p}}\right)=\#\left(\Gamma_{\mathfrak{p}}\right), \quad \operatorname{dim}_{\mathbb{R}}\left(\mathfrak{j}_{\mathfrak{p}_{u}}\right) / 4 \in \mathbb{N} .
$$

We complexify this decomposition and obtain

$$
\mathfrak{o}_{\mathfrak{p}}=\mathfrak{j}_{\mathfrak{p}} \oplus \mathfrak{z}_{\mathfrak{p}}^{\mathbb{C}} \quad \operatorname{dim}_{\mathbb{C}}\left(\mathfrak{z}_{\mathfrak{p}}^{\mathbb{C}}\right)=\#\left(\Gamma_{\mathfrak{p}}\right), \quad \operatorname{dim}\left(\mathfrak{j}_{\mathfrak{p}}\right) / 4 \in \mathbb{N}
$$

The following conditions for integrability are obvious (and well known):

Proposition 3.10. Let $C: \mathfrak{p}_{u} \rightarrow \mathfrak{p}_{u}$ be a complex structure on $\mathfrak{p}_{u}$. Let $C: \mathfrak{p} \rightarrow \mathfrak{p}$ be the complexification of $C$. Let $\mathfrak{a}^{+}$and $\mathfrak{a}^{-}$be the $\mathrm{i}$ and $(-\mathrm{i})$-eigen subspaces of $C$ in $\mathfrak{p}$. Then the following conditions are equivalent

$$
\begin{gathered}
\forall X, Y \in \mathfrak{p}_{u}[C(X), C(Y)]_{\mathfrak{p}_{u}}-[X, Y]_{\mathfrak{p}_{u}}-C\left([C(X), Y]_{\mathfrak{p}_{u}}\right)-C\left([X, C(Y)]_{\mathfrak{p}_{u}}\right)=0 ; \\
\forall X, Y \in \mathfrak{a}^{ \pm} \quad[X, Y]_{\mathfrak{p}} \in \mathfrak{a}^{ \pm} .
\end{gathered}
$$

Proposition 3.11. Let $\kappa \in \operatorname{Aut}(\mathfrak{g})$ be an automorphism of Lie algebras, such that $\kappa(\mathfrak{p})=\mathfrak{p}$, $\kappa(\mathfrak{k})=\mathfrak{k}$. Let $\mathfrak{a} \subset \mathfrak{p}$ be a subspace, such that for $X, Y \in \mathfrak{a}$ we have $[X, Y]_{\mathfrak{p}} \in \mathfrak{a}$. Then

$$
\forall X, Y \in \kappa(\mathfrak{a}) \quad[X, Y]_{\mathfrak{p}} \in \kappa(\mathfrak{a}) .
$$

3.2.2. The complex structure $I$. We define a complex structure $I: \mathfrak{p}_{u} \rightarrow \mathfrak{p}_{u}$ (with complexification $I: \mathfrak{p} \rightarrow \mathfrak{p})$ by (31) and (25))

$$
\begin{gathered}
I\left(\mathfrak{w}_{\mathfrak{p}}\right)=\mathfrak{z}_{\mathfrak{p}} \quad I\left(\mathfrak{j}_{\mathfrak{p}_{u}}\right)=\mathfrak{j}_{\mathfrak{p}_{u}} \quad I\left(\mathfrak{j}_{\mathfrak{p}}\right)=\mathfrak{j}_{\mathfrak{p}} \\
\forall \alpha \in \Delta_{\mathfrak{p}}^{+} \quad I\left(E_{\alpha}\right)=\mathrm{i} E_{\alpha}, \quad I\left(E_{-\alpha}\right)=-\mathrm{i} E_{-\alpha} .
\end{gathered}
$$

We can rewrite (27) and (23), using Corollary 3.8 and (32), as follows:

$$
\mathfrak{p}=\mathfrak{j}_{\mathfrak{p}} \oplus \sum_{\gamma \in \Gamma_{\mathfrak{p}}}\left(\mathcal{V}_{\gamma} \oplus g l_{\gamma}(2)\right) \quad \mathfrak{k}=\mathfrak{o}_{\mathfrak{k}} \oplus \sum_{\gamma \in \Gamma_{\mathfrak{k}}}\left(\mathcal{V}_{\gamma} \oplus s l_{\gamma}(2)\right),
$$

where $g l_{\gamma}(2)$ is as in Definition 3.1. 
We have

$$
\begin{aligned}
\mathfrak{p}_{s}^{+} & =\sum_{\gamma \in \Gamma_{\mathfrak{p}}}\left(\mathbb{C} P_{\gamma}+\mathbb{C} E_{\gamma}+\mathcal{V}_{\gamma}^{+}\right) \\
\mathfrak{p}_{s}^{-} & =\sum_{\gamma \in \Gamma_{\mathfrak{p}}}\left(\mathbb{C} Q_{\gamma}+\mathbb{C} E_{-\gamma}+\mathcal{V}_{\gamma}^{-}\right)=\tau\left(\mathfrak{p}_{s}^{+}\right) \\
\mathfrak{p}_{s} & =\mathfrak{p}_{s}^{+} \oplus \mathfrak{p}_{s}^{-} \Rightarrow \mathfrak{p}=\mathfrak{j}_{\mathfrak{p}} \oplus \mathfrak{p}_{s}, \quad \mathfrak{g}=\mathfrak{j}_{\mathfrak{p}} \oplus \mathfrak{p}_{s} \oplus \mathfrak{k} .
\end{aligned}
$$

Obviously for any choice of $X, Y \in \mathfrak{p}_{s}^{ \pm}$we have $[X, Y]_{\mathfrak{p}} \in \mathfrak{p}_{s}^{ \pm}$. We have proved:

Proposition 3.12. Let $\mathfrak{a} \subset \mathfrak{j}_{\mathfrak{p}}$ be an arbitrary subspace of $\mathfrak{j}_{\mathfrak{p}}$. Then for any $X, Y \in \mathfrak{p}_{s}^{ \pm}+\mathfrak{a}$ we have $[X, Y]_{\mathfrak{p}} \in \mathfrak{p}_{s}^{ \pm}+\mathfrak{a}$.

If we denote by $\mathfrak{p}^{+}$and $\mathfrak{p}^{-}$the $\mathrm{i}$ and $(-\mathrm{i})$-eigen spaces of $I$, then (see (133) $) \mathfrak{p}^{ \pm}=\mathfrak{p}_{s}^{ \pm}+\left(\mathfrak{p}^{ \pm} \cap \mathfrak{j}_{\mathfrak{p}}\right)$ and from Proposition 3.12 it follows

$$
\forall X, Y \in \mathfrak{p}^{ \pm} \quad[X, Y]_{\mathfrak{p}} \in \mathfrak{p}^{ \pm} .
$$

3.2.3. The complex structure $J$. For each $\gamma \in \Gamma$ we have an inner automorphism 6 of $\mathfrak{g}$

$$
\mathbf{c}_{\gamma}=\exp \left(\frac{\pi}{2} a d X_{\gamma}\right) \quad \gamma \in \Gamma
$$

where $X_{\gamma}$ is given in Definition 2.5.

We recall (see [6]) some properties of the inner automorphisms $\mathbf{c}_{\gamma}$.

Lemma 3.13. Let $\gamma, \delta \in \Gamma$. Let $\alpha \in \Phi_{\delta}^{+}$and $H \in \mathfrak{h}$. Then

$$
\begin{gathered}
\mathbf{c}_{\gamma} \circ \tau=\tau \circ \mathbf{c}_{\gamma}, \quad \mathbf{c}_{\gamma} \circ \mathbf{c}_{\delta}=\mathbf{c}_{\delta} \circ \mathbf{c}_{\gamma} ; \\
\delta \neq \gamma \quad \Rightarrow \quad \mathbf{c}_{\gamma}\left(\mathcal{V}_{\delta}^{+}\right)=\mathcal{V}_{\delta}^{+} \\
\delta=\gamma \Rightarrow \mathbf{c}_{\gamma}\left(E_{\alpha}\right)=\frac{\sqrt{2}}{2}\left(E_{\alpha}+\overline{\rho_{\gamma}} N_{\gamma,-\alpha} E_{\alpha-\gamma}\right) \\
\mathbf{c}_{\gamma}\left(E_{\gamma}\right)=E_{\gamma}+\mathrm{i} \overline{\rho_{\gamma}}\left(Y_{\gamma}-W_{\gamma}\right)=\overline{\rho_{\gamma}}\left(X_{\gamma}-\mathrm{i} W_{\gamma}\right) \\
\mathbf{c}_{\gamma}(H)=H+\mathrm{i} \gamma(H)\left(Y_{\gamma}+W_{\gamma}\right) .
\end{gathered}
$$

Let us define the Cayley transform

$$
\mathbf{c}=\prod_{\gamma \in \Gamma} \mathbf{c}_{\gamma}
$$

where multiplication is the composition of automorphisms (which commute).

\footnotetext{
${ }^{6}$ Rather a circle of automorphisms depending on the parameter $\rho_{\gamma}$ (see Definition 2.5)
} 
By an easy computation, using Lemma 3.13, one can derive (we recall that for $\gamma \in \Gamma_{\mathfrak{p}}$ we denote $P_{\gamma}=W_{\gamma}-\mathrm{i} I W_{\gamma}, Q_{\gamma}=W_{\gamma}+\mathrm{i} I W_{\gamma}$ and that $\left.I W_{\gamma} \in \mathfrak{o}\right)$

$$
\begin{array}{r}
\forall \gamma \in \Gamma \quad \mathbf{c}\left(\mathcal{V}_{\gamma}^{+}\right)=\sum_{\alpha \in \Phi_{\gamma}^{+}} \mathbb{C}\left(E_{\alpha}+\overline{\rho_{\gamma}} N_{\gamma,-\alpha} E_{\alpha-\gamma}\right) \quad \mathbf{c}\left(s l_{\gamma}(2)\right)=s l_{\gamma}(2) \\
\forall \gamma \in \Gamma_{\mathfrak{p}} \quad \mathbf{c}\left(\operatorname{span}\left\{P_{\gamma}, E_{\gamma}\right\}\right)=\operatorname{span}\left\{E_{\gamma}-\mathrm{i} \overline{\rho_{\gamma}} Q_{\gamma}, P_{\gamma}-\mathrm{i} \overline{\rho_{\gamma}} E_{-\gamma}\right\},
\end{array}
$$

hence by (34) we obtain immediately

$$
\mathbf{c}(\mathfrak{k})=\mathfrak{k}, \quad \mathbf{c}(\mathfrak{p})=\mathfrak{p} .
$$

Furthermore, using (45) we obtain (see (35) also)

$$
\begin{aligned}
\mathbf{c}\left(\mathfrak{p}_{s}^{+}\right)= & \sum_{\gamma \in \Gamma_{\mathfrak{p}}} \sum_{\alpha \in \Phi_{\gamma}^{+}} \mathbb{C}\left(E_{\alpha}+\overline{\rho_{\gamma}} N_{\gamma,-\alpha} E_{\alpha-\gamma}\right) \\
& +\sum_{\gamma \in \Gamma_{\mathfrak{p}}} \mathbb{C}\left(E_{\gamma}-\mathrm{i} \overline{\rho_{\gamma}} Q_{\gamma}\right)+\sum_{\gamma \in \Gamma_{\mathfrak{p}}} \mathbb{C}\left(P_{\gamma}-\mathrm{i} \overline{\rho_{\gamma}} E_{-\gamma}\right) .
\end{aligned}
$$

Now using $\mathfrak{p}_{s}^{-}=\tau\left(\mathfrak{p}_{s}^{+}\right)$and $\tau \circ \mathbf{c}=\mathbf{c} \circ \tau$ we see that

$$
\begin{aligned}
\mathbf{c}\left(\mathfrak{p}_{s}^{-}\right)= & \sum_{\gamma \in \Gamma_{\mathfrak{p}}} \sum_{\alpha \in \Phi_{\gamma}^{+}} \mathbb{C}\left(E_{-\alpha}+\rho_{\gamma} N_{\gamma,-\alpha} E_{-\alpha+\gamma}\right) \\
& +\sum_{\gamma \in \Gamma_{\mathfrak{p}}} \mathbb{C}\left(E_{-\gamma}-\mathrm{i} \rho_{\gamma} P_{\gamma}\right)+\sum_{\gamma \in \Gamma_{\mathfrak{p}}} \mathbb{C}\left(Q_{\gamma}-\mathrm{i} \rho_{\gamma} E_{\gamma}\right) .
\end{aligned}
$$

Now we define a complex structure $J: \mathfrak{p}_{u} \rightarrow \mathfrak{p}_{u}$ with complexification $J: \mathfrak{p} \rightarrow \mathfrak{p}$ such that (we recall that $\operatorname{dim}_{\mathbb{R}}\left(\mathfrak{j}_{\mathfrak{p}_{u}}\right) / 4 \in \mathbb{N}$ and $I\left(\mathfrak{j}_{\mathfrak{p}_{u}}\right)=\mathfrak{j}_{\mathfrak{p}_{u}}$ )

$$
\begin{gathered}
J\left(\mathfrak{j}_{\mathfrak{p}_{u}}\right)=\mathfrak{j}_{\mathfrak{p}_{u}} \quad J_{\mid \mathfrak{j}_{\mathfrak{p}_{u}}} \circ I_{\mid \mathfrak{j}_{\mathfrak{p}_{u}}}=-I_{\mid \mathfrak{j}_{\mathfrak{p}_{u}}} \circ J_{\mid \mathfrak{j}_{\mathfrak{p}_{u}}} \\
J\left(E_{\gamma}\right)=\overline{\rho_{\gamma}} Q_{\gamma} \quad J\left(E_{-\gamma}\right)=-\rho_{\gamma} P_{\gamma} \quad \gamma \in \Gamma_{\mathfrak{p}} \\
J\left(E_{\alpha}\right)=\mathrm{i} N_{\gamma,-\alpha} \overline{\rho_{\gamma}} E_{\alpha-\gamma} \quad J\left(E_{-\alpha}\right)=-\mathrm{i} N_{\gamma,-\alpha} \rho_{\gamma} E_{\gamma-\alpha} \quad \alpha \in \Phi_{\gamma}^{+}, \gamma \in \Gamma_{\mathfrak{p}} .
\end{gathered}
$$

It is easy to check that the complex structures given in (16), (17) define the same hypercomplex structure as (49).

We see that $J\left(\mathfrak{p}_{s}\right)=\mathfrak{p}_{s}$ and by (33) we compute $J_{\mid \mathfrak{p}_{s}} \circ I_{\mid \mathfrak{p}_{s}}=-I_{\mid \mathfrak{p}_{s}} \circ J_{\mid \mathfrak{p}_{s}}$. Therefore $J \circ I=-I \circ J$.

Let $\mathfrak{p}_{J}^{+}$and $\mathfrak{p}_{J}^{-}$be the $\mathrm{i}$ and $(-\mathrm{i})$-eigen spaces of $J$. Comparing (49) and (47), (48), we see that

$$
\mathfrak{p}_{J}^{ \pm}=\mathbf{c}\left(\mathfrak{p}_{s}^{ \pm}\right)+\left(\mathfrak{p}_{J}^{ \pm} \cap \mathfrak{j}_{\mathfrak{p}}\right)=\mathbf{c}\left(\mathfrak{p}_{s}^{ \pm}+\left(\mathfrak{p}_{J}^{ \pm} \cap \mathfrak{j}_{\mathfrak{p}}\right)\right) .
$$

Now Propositions 3.11, 3.12 and formula (46) imply

$$
\forall X, Y \in \mathfrak{p}_{J}^{ \pm} \quad[X, Y]_{\mathfrak{p}} \in \mathfrak{p}_{J}^{ \pm} .
$$


3.2.4. ad(k)-invariance of $I$ and $J$. From the decomposition (34) and the definitions of $I$ and $J$ (formulas (33) and (49)) we see that $\mathfrak{j}_{\mathfrak{p}}, \mathcal{V}_{\gamma}$ and $g l_{\gamma}(2)$ for $\gamma \in \Gamma_{\mathfrak{p}}$ are $I$ and $J$ invariant. We have (see (16) also)

$$
I_{\mid v_{\gamma}}=a d\left(2 W_{\gamma}\right)\left|\mathcal{v}_{\gamma}, \quad J_{\mid v_{\gamma}}=-a d\left(2 Y_{\gamma}\right)\right| v_{\gamma}, \quad \gamma \in \Gamma_{\mathfrak{p}}
$$

It turns out that the subspaces $\mathcal{V}_{\gamma}$ and $g l_{\gamma}(2)$ are $a d(\mathfrak{k})$-invariant as well, which is a straightforward consequence of (28) and (29), more precisely one may compute:

$$
\forall \gamma \in \Gamma_{\mathfrak{p}}, \forall X \in \mathfrak{k} \quad \operatorname{ad}(X)\left(\mathcal{V}_{\gamma}\right) \subset \mathcal{V}_{\gamma}, \quad \operatorname{ad}(X)\left(g l_{\gamma}(2)\right)=\{0\} .
$$

Lemma 3.14. The complex structures $I, J$ give a hypercomplex structure on $\mathfrak{p}_{u}$, i.e. for $X, Y \in \mathfrak{p}_{u}$ we have

$$
\begin{gathered}
{[J(X), J(Y)]_{\mid \mathfrak{p}_{u}}-[X, Y]_{\mid \mathfrak{p}_{u}}-J\left([X, J(Y)]_{\mid \mathfrak{p}_{u}}\right)-J\left([J(X), Y]_{\mid \mathfrak{p}_{u}}\right)=0} \\
{[I(X), I(Y)]_{\mid \mathfrak{p}_{u}}-[X, Y]_{\mid \mathfrak{p}_{u}}-I\left([X, I(Y)]_{\mid \mathfrak{p}_{u}}\right)-I\left([I(X), Y]_{\mid \mathfrak{p}_{u}}\right)=0}
\end{gathered}
$$

Moreover for $X \in \mathfrak{k}_{u}, Y \in \mathfrak{p}_{u}$ we have

$$
I \circ \operatorname{ad}(X)(Y)=\operatorname{ad}(X) \circ I(Y), \quad J \circ \operatorname{ad}(X)(Y)=\operatorname{ad}(X) \circ J(Y) .
$$

Proof. Vanishing of the Nijenhuis tensors follows from Lemma 3.10 and formulas (38), (50).

Now we go to the proof of (53). Using (151) and (52) we readily deduce that for $\gamma \in \Gamma_{\mathfrak{p}}$, $X \in \mathfrak{k}$ we have

$$
\begin{gathered}
(\operatorname{ad}(X) \circ I)_{\mid \mathcal{V}_{\gamma}}=(I \circ \operatorname{ad}(X))_{\mid \mathcal{V}_{\gamma}} \quad(\operatorname{ad}(X) \circ J)_{\mid \mathcal{V}_{\gamma}}=(J \circ \operatorname{ad}(X))_{\mid \mathcal{V}_{\gamma}}, \\
(\operatorname{ad}(X) \circ I)_{\mid g l_{\gamma}(2)}=(I \circ \operatorname{ad}(X))_{\mid g l_{\gamma}(2)}=(\operatorname{ad}(X) \circ J)_{\mid g l_{\gamma}(2)}=(J \circ \operatorname{ad}(X))_{\mid g l_{\gamma}(2)}=0 .
\end{gathered}
$$

Finally, from (29) and since $\mathfrak{j}_{\mathfrak{p}} \subset \mathfrak{h}_{\mathfrak{p}}$ we see that

$$
\forall \alpha \in \Delta_{\mathfrak{k}} \quad \alpha_{\mid \mathfrak{j}_{\mathfrak{p}}}=0 \quad \Rightarrow \quad \forall X \in \mathfrak{k} \quad \operatorname{ad}(X)_{\left.\right|_{\mathfrak{j} \mathfrak{p}}}=0 .
$$

Hence, $\mathfrak{j}_{\mathfrak{p}}$ being $I$ and $J$ invariant, for each $X \in \mathfrak{k}$ we get

$$
(\operatorname{ad}(X) \circ I)_{\mid \mathfrak{j}_{\mathfrak{p}}}=(I \circ \operatorname{ad}(X))_{\mid \mathfrak{j}_{\mathfrak{p}}}=(\operatorname{ad}(X) \circ J)_{\mid \mathfrak{j}_{\mathfrak{p}}}=(J \circ \operatorname{ad}(X))_{\left.\right|_{\mathfrak{j}_{\mathfrak{p}}}}=0 .
$$

Using the decomposition (34) of $\mathfrak{p}$, we see that for all $X \in \mathfrak{k}, \quad Y \in \mathfrak{p}$ we have

$$
I \circ \operatorname{ad}(X)(Y)=\operatorname{ad}(X) \circ I(Y) \quad J \circ \operatorname{ad}(X)(Y)=\operatorname{ad}(X) \circ J(Y) .
$$

Recalling that $I$ and $J$ commute with $\tau$, we obtain (53).

So we are given a pair of groups $\left(\mathbf{U}, \mathbf{K}_{u}\right)$ with connected $\mathbf{K}_{u}$ associated with a hypercomplex pair $\left(\mathfrak{u}, \mathfrak{k}_{u}\right)$. We constructed anti-commuting complex structures $I$ and $J$ on $\mathfrak{p}_{u}$, which have the properties listed in Lemma 3.14 and also we have the reductivity condition $\left[\mathfrak{k}_{u}, \mathfrak{p}_{u}\right] \subset \mathfrak{p}_{u}$. Then, it is known (see e.g. [10], Ch. X), that $I$ and $J$ determine left-invariant, integrable complex structures on the coset space $\mathbf{U} / \mathbf{K}_{u}$, which thus becomes a compact, homogeneous, hypercomplex space. 
Definition 3.15. Let $\left(\mathfrak{u}, \mathfrak{k}_{u}\right)$ be a hypercomplex pair and let $\left(\mathbf{U}, \mathbf{K}_{u}\right)$ be a pair of groups associated with it. Let $\left(I_{M}, J_{M}\right)$ be a LIHCS on $M=\mathbf{U} / \mathbf{K}_{u}$ and let $\pi: \mathbf{U} \rightarrow M$ be the natural projection. We shall say that the homogeneous hypercomplex space $\left(M, I_{M}, J_{M}\right)$ is associated with the hypercomplex pair $\left(\mathfrak{u}, \mathfrak{k}_{u}\right)$ if the complex structures $I, J$ on $\mathfrak{p}_{u}$ constructed above satisfy:

$$
I=\left((\mathrm{d} \pi)_{\mid \mathfrak{p}_{u}}\right)^{-1} \circ I_{M}(o) \circ(\mathrm{d} \pi)_{\mid \mathfrak{p}_{u}}, \quad J=\left((\mathrm{d} \pi)_{\mid \mathfrak{p}_{u}}\right)^{-1} \circ J_{M}(o) \circ(\mathrm{d} \pi)_{\mid \mathfrak{p}_{u}} .
$$

In the next section we prove that any $\mathbf{U}$ - left-invariant, hypercomplex structure on a coset space $\mathbf{U} / \mathbf{K}_{u}$ is associated with the hypercomplex pair $\left(\mathfrak{u}, \mathfrak{k}_{u}\right)$.

\section{NeCESSARY CONDITIONS}

4.1. Introduction. In this section we shall discuss a coset space $M=\mathbf{U} / \mathbf{K}_{u}$, where $\mathbf{U}$ is a compact, connected Lie group and $\mathbf{K}_{u}$ is a closed subgroup. We shall assume that $\mathbf{U}$ acts effectively on $M$. Furthermore we shall assume that there are two left-invariant complex structures $I_{M}$ and $J_{M}$, which anti-commute, on $M$. By left-invariant we mean that all the left translations $l_{u}: M \rightarrow M$, where $u$ varies through $\mathbf{U}$, are holomorphic with respect to both $I_{M}$ and $J_{M}$.

We are going to prove that $M$ is associated to a hypercomplex pair.

4.2. $M$ as a coset space of a complex Lie group. So, we are given a compact coset space $M=\mathbf{U} / \mathbf{K}_{u}$ and two complex structures $I_{M}$ and $J_{M}$ on it. Let $\mathbf{G}_{I 0}$ be the identity component of the group of all biholomorphisms of the complex manifold $\left(M, I_{M}\right)$. By results of Bochner and Montgomery in [3] and [4] it follows that one can define a complex structure on $\mathbf{G}_{I 0}$, such that $\mathbf{G}_{I 0}$ becomes a complex Lie group and the action on the complex manifold $\left(M, I_{M}\right)$

$$
\mathbf{G}_{I 0} \times M \rightarrow M \quad(f, m) \in \mathbf{G}_{I} \times M \mapsto f(m) \in M
$$

is holomorphic.

Let $\pi$ be the natural projection

$$
\pi: \mathbf{U} \rightarrow \mathbf{U} / \mathbf{K}_{u}=M \quad \pi(e)=o .
$$

We are given, that $\mathbf{U}$ is a compact, connected subgroup of $\mathbf{G}_{I 0}$ which acts effectively and transitively on $M$. Let $\mathfrak{u}$ and $\mathfrak{g}_{I 0}$ be the Lie algebras of $\mathbf{U}$ and $\mathbf{G}_{I 0}$, respectively. Since $\mathfrak{u}$ is compact, then we have the decomposition $\mathfrak{u}=\mathfrak{c}_{u} \oplus \mathfrak{u}_{s}$ (see (1) ). We recall [7, page 132] that the semi-simple part $\mathfrak{u}_{s}$ is a compact Lie algebra also. We denote by $\mathbf{U}_{s}$ the connected subgroup of $\mathbf{U}$ with Lie algebra $\mathfrak{u}_{s}$. Since $\mathfrak{u}_{s}$ is a compact, semi-simple Lie algebra, then (see [7, page 133]) $\mathbf{U}_{s}$ is a compact Lie group, hence it is a closed, compact, semi-simple subgroup of $\mathbf{G}_{I 0}$.

Lemma 4.1. The real subalgebra $\mathfrak{u}_{s}$ of the complex Lie algebra $\mathfrak{g}_{I 0}$ satisfies

$$
\mathfrak{u}_{s} \cap \mathfrak{i u}_{s}=\{0\} .
$$

In particular the subspace $\mathfrak{u}_{s}+\mathfrak{i u}_{s} \subset \mathfrak{g}_{I 0}$ is a complex semi-simple subalgebra of $\mathfrak{g}_{I 0}$, which is a complexification of $\mathfrak{u}_{s}$, we shall denote it by $\mathfrak{g}_{s}$. 
Proof. Let $\mathbf{G}_{m}$ be a maximal connected semi-simple subgroup of $\mathbf{G}_{I 0}$, which contains $\mathbf{U}_{s}$. Actually (see the proof of Theorem 2.3 in [16]), $\mathbf{G}_{m}$ is generated by the semi-simple part of a Levi-decomposition of the complex Lie algebra of $\mathbf{G}_{I 0}$. Therefore $\mathbf{G}_{m}$ is a complex semi-simple Lie group and $\mathbf{U}_{s}$ is its connected, compact, real Lie subgroup. Let $\mathfrak{g}_{m}$ be the complex Lie algebra of $\mathbf{G}_{m}$. Then the Lie algebra $\mathfrak{u}_{s}$ of $\mathbf{U}_{s}$ is a real subalgebra of $\mathfrak{g}_{m}$.

Now $\mathbf{G}_{m}$ is a connected complex semi-simple Lie group, therefore it is semi-simple as a real Lie group also. Let us take any compact real form $\mathfrak{b}$ of $\mathfrak{g}_{m}$. Then the subgroup $\mathbf{B}$ of $\mathbf{G}_{m}$ generated by $\mathfrak{b}$ is a maximal compact subgroup. Let $\mathbf{B}_{u}$ be a maximal compact subgroup of $\mathbf{G}_{m}$, containing $\mathbf{U}_{s}$, let the Lie algebra of $\mathbf{B}_{u}$ be $\widetilde{\mathfrak{u}}$. The subgroup $\mathbf{B}_{u}$ is connected (all maximal compact subgroups in a semi-simple connected group are connected and conjugate to each other- see [7, page 356]). Therefore there exists an element $g \in \mathbf{G}_{m}$ such that $\alpha_{g}(\mathbf{B})=g \mathbf{B} g^{-1}=\mathbf{B}_{u}$, where by $\alpha_{g}: \mathbf{G}_{m} \rightarrow \mathbf{G}_{m}$ we denote the conjugation of $\mathbf{G}_{m}$ with the element $g$. Since $\mathbf{G}_{m}$ is a complex group then $\left(\mathrm{d} \alpha_{g}\right)_{e}=A d(g): \mathfrak{g}_{m} \rightarrow \mathfrak{g}_{m}$ is an automorphism of the complex Lie algebra $\mathfrak{g}_{m}$. Since $\alpha_{g}(\mathbf{B})=\mathbf{B}_{u}$ then, obviously, $A d(g)(\mathfrak{b})=\widetilde{\mathfrak{u}}$. Since $\mathfrak{b}$ is a compact form, then $\widetilde{\mathfrak{u}}$ is a compact form of $\mathfrak{g}_{m}$ and $\widetilde{\mathfrak{u}} \cap \tilde{i} \mathfrak{u}=\{0\}$. On the other hand $\mathfrak{u}_{s} \subset \widetilde{\mathfrak{u}}$ and (56) follows.

Let us denote

$$
\mathfrak{g}_{I}=\mathfrak{u}+\mathfrak{i} \mathfrak{u} \subset \mathfrak{g}_{I 0} .
$$

If we denote $\mathfrak{c}_{I}=\mathfrak{c}_{u}+\mathrm{i} \mathfrak{c}_{u} \subset \mathfrak{g}_{I}$ then $\mathfrak{c}_{I}$ is abelian and obviously $\left[\mathfrak{c}_{I}, \mathfrak{g}_{s}\right] \subset\{0\}$. Therefore, since $\mathfrak{g}_{s}$ is semi-simple, we have $\mathfrak{c}_{I} \cap \mathfrak{g}_{s}=\{0\}$ and we may write

$$
\mathfrak{g}_{I}=\mathfrak{c}_{I} \oplus \mathfrak{g}_{s} \quad \mathfrak{c}_{I}=\mathfrak{c}_{u}+\mathrm{i} \mathfrak{c}_{u} .
$$

Let $\mathbf{G}_{I}$ be the connected subgroup of $\mathbf{G}_{I 0}$ generated by the subalgebra $\mathfrak{g}_{I} \subset \mathfrak{g}_{I 0}$. Obviously $\mathbf{G}_{I} \supset \mathbf{U}$ and therefore $\mathbf{G}_{I}$ acts transitively on $M=\mathbf{U} / \mathbf{K}_{u}$. Hence the complex manifold $\left(M, I_{M}\right)$ is biholomorphic with the complex coset space $\mathbf{G}_{I} / \mathbf{L}$, where (we recall that $\pi(e)=o$ )

$$
\mathbf{L}=\left\{g \in \mathbf{G}_{I} \mid g(o)=o\right\} .
$$

Obviously $\mathbf{K}_{u}=\mathbf{U} \cap \mathbf{L}$. Let us denote by $\mathfrak{l}$ the complex subalgebra of $\mathfrak{g}_{I}$, corresponding to the complex subgroup $\mathbf{L}$ and by $\mathfrak{k}_{u}$ the subalgebra of $\mathfrak{u}$, corresponding to the subgroup $\mathbf{K}_{u}$.

Let $p$ be the natural projection

$$
p: \mathbf{G}_{I} \rightarrow M \simeq \mathbf{G}_{I} / \mathbf{L} .
$$

Since $p \circ$ in $=\pi$, where $i n: \mathbf{U} \rightarrow \mathbf{G}_{I}$ is the embedding of $\mathbf{U}$ in $\mathbf{G}_{I}$, then the restriction of $\mathrm{d} p$ to $\mathfrak{u}$ is equal to $\mathrm{d} \pi: \mathfrak{u} \rightarrow T_{o}(M)$, i. e.

$$
\forall X \in \mathfrak{u} \quad \mathrm{d} p(X)=\mathrm{d} \pi(X) .
$$

Furthermore, since the projection $p$ is holomorphic then

$$
\forall X \in \mathfrak{g}_{I} \quad \mathrm{~d} p(\mathrm{i} X)=I_{M}(\mathrm{~d} p(X))=\operatorname{id} p(X) .
$$

Since $\mathbf{K}_{u}=\mathbf{U} \cap \mathbf{L}$ then $\mathfrak{k}_{u}=\mathfrak{u} \cap \mathfrak{l}$. Furthermore

$$
\mathfrak{k} \mathfrak{e r}(\mathrm{d} \pi)=\mathfrak{k}_{u} \quad \mathfrak{k} \mathfrak{e r}(\mathrm{d} p)=\mathfrak{l} \quad \mathfrak{u} \cap \mathfrak{l}=\mathfrak{k}_{u} .
$$


Therefore the subspace

$$
\mathfrak{p}_{u}=\left\{X \in \mathfrak{u} \mid \quad \forall Y \in \mathfrak{k}_{u}\langle X, Y\rangle=0\right\}
$$

satisfies

$$
\mathfrak{u}=\mathfrak{k}_{u} \oplus \mathfrak{p}_{u} .
$$

Since $\mathfrak{k}_{u}$ is $A d\left(\mathbf{K}_{u}\right)$-invariant then obviously $\mathfrak{p}_{u}$ is $A d\left(\mathbf{K}_{u}\right)$-invariant:

$$
\operatorname{Ad}\left(\mathbf{K}_{u}\right)\left(\mathfrak{p}_{u}\right) \subset \mathfrak{p}_{\mathfrak{u}} \Rightarrow\left[\mathfrak{k}_{u}, \mathfrak{p}_{u}\right] \subset \mathfrak{p}_{u} .
$$

Let $\mathfrak{g}$ and $\mathfrak{k}$ be the complexifications of $\mathfrak{u}, \mathfrak{k}_{u}$ and let $\tau$ be the conjugation of $\mathfrak{g}$, such that $\mathfrak{u}=\mathfrak{g}^{\tau}$. We shall denote the center of $\mathfrak{g}$ by $\mathfrak{c}$.

Obviously, we have

$$
\mathfrak{k}=\mathfrak{k}_{u}+\mathfrak{i k}_{u} \subset \mathfrak{g} \quad \mathfrak{k}_{u} \cap \mathfrak{i k}_{u}=\{0\} .
$$

Remark 4.2. Let us summarize. We have $\mathfrak{g}_{I 0}$ - the complex Lie algebra of the complex Lie group $\mathbf{G}_{I 0}$ (the identity component of the group of all biholomorphisms of the complex manifold $\left.\left(M, I_{M}\right)\right)$. In $\mathfrak{g}_{I 0}$ live our main actors $\mathfrak{u} \subset \mathfrak{g}_{I 0}, \mathfrak{k}_{u} \subset \mathfrak{g}_{I 0}$ which are the real Lie algebras of $\mathbf{U}$ and $\mathbf{K}_{u}$. And we denote by $\mathfrak{g}_{I}$ the complex subalgebra of $\mathfrak{g}_{I 0}$ generated by $\mathfrak{u}$, $i$. e. $\mathfrak{g}_{I}=\mathfrak{u}+\mathrm{i} \mathfrak{u} \subset \mathfrak{g}_{I 0}, \mathbf{G}_{I} \subset \mathbf{G}_{I 0}$ is the corresponding complex Lie group. By $\mathfrak{g}$ and $\mathfrak{k} \subset \mathfrak{g}$ we denote the usual complexifications of the real Lie algebras $\mathfrak{u}$ and $\mathfrak{k}_{u}$, and $\tau$ is the conjugation.

Since $\mathfrak{u}$ is compact we can decompose it as direct sum of center and semisimple part: $\mathfrak{u}=\mathfrak{c}_{u} \oplus \mathfrak{u}_{s}$. From Lemma 4.1] we see that the complex subalgebra of $\mathfrak{g}$ generated by $\mathfrak{u}_{s}$ is isomorphic to the subalgebra $\mathfrak{g}_{s}=\mathfrak{u}_{s}+\mathfrak{i u}_{s} \subset \mathfrak{g}_{I}$ of $\mathfrak{g}_{I}$. Therefore we can identify these two algebras (the identification is: for $X, Y \in \mathfrak{u}_{s} X+\mathrm{i} Y \in \mathfrak{g} \mapsto X+\mathrm{i} Y \in \mathfrak{g}_{I}$ ), and we shall denote them by the common notation: $\mathfrak{g}_{s}$. With this convention we have $\mathfrak{g}=\mathfrak{c} \oplus \mathfrak{g}_{s}$, where $\mathfrak{c}$ is the center of $\mathfrak{g}$.

Denoting by $\tau_{s}$ the restriction of $\tau$ to $\mathfrak{g}_{s}$, we have

$$
\forall Y, Z \in \mathfrak{u} \quad \tau_{s}(Y+\mathrm{i} Z)=Y-\mathrm{i} Z, \quad \tau(\mathfrak{c})=\mathfrak{c} .
$$

We represented our homogeneous space $M$ as complex factor $\mathbf{G}_{I} / \mathbf{L}$ and denoted by $\mathfrak{l}$ the complex subalgebra of $\mathfrak{g}_{I}$, corresponding to the complex subgroup $\mathbf{L} \subset \mathbf{G}_{I}$.

The Lie algebras $\mathfrak{g}_{I} \supset \mathfrak{l}$ are auxiliary and will be used to prove the regularity of $\mathfrak{k}, \mathfrak{p}$.

4.3. Construction of $\mathbf{I}, \mathbf{J}$. Let us denote by $\mathfrak{p}$ the complex subspace of $\mathfrak{g}_{I}$, generated by $\mathfrak{p}_{\mathfrak{u}}$ :

$$
\mathfrak{p}=\mathfrak{p}_{u}+\mathrm{ip} \mathfrak{p}_{u} \subset \mathfrak{g}_{I}
$$

We shall prove soon (Corollary 4.5) that the sum above is actually direct sum of real vector spaces, i. e. that $\mathfrak{p}$ is a complexification of $\mathfrak{p}_{u}$, therefore we may regard $\mathfrak{p}$ as a subspace of $\mathfrak{g}$.

We define complex structures $I, J$ on $\mathfrak{p}_{u}$, using the complex structures $I_{M}(o), J_{M}(o)$ on $T_{o}(M)$, as follows

$$
I=\left((\mathrm{d} \pi)_{\mid \mathfrak{p}_{u}}\right)^{-1} \circ I_{M}(o) \circ(\mathrm{d} \pi)_{\mid \mathfrak{p}_{u}}, \quad J=\left((\mathrm{d} \pi)_{\mid \mathfrak{p}_{u}}\right)^{-1} \circ J_{M}(o) \circ(\mathrm{d} \pi)_{\mid \mathfrak{p}_{u}} .
$$


Lemma 4.3. The complex structure $I$ on the real vector space $\mathfrak{p}_{u}$ can be uniquely extended to a complex structure on the complex vector space $\mathfrak{p}$. We shall denote this extension by the same letter $I: \mathfrak{p} \rightarrow \mathfrak{p}$.

Proof. Let us take any element $Z \in \mathfrak{p}$. Let us assume that we have two representations of $Z$ of the type

$$
Z=X_{1}+\mathrm{i} Y_{1}=X_{2}+\mathrm{i} Y_{2}, \quad X_{1}, X_{2}, Y_{1}, Y_{2} \in \mathfrak{p}_{u}
$$

therefore

$$
\begin{gathered}
\mathrm{d} p\left(X_{1}+\mathrm{i} Y_{1}\right)=\mathrm{d} p\left(X_{2}+\mathrm{i} Y_{2}\right) \Rightarrow \mathrm{d} p\left(X_{1}\right)+\mathrm{id} p\left(Y_{1}\right)=\mathrm{d} p\left(X_{2}\right)+\mathrm{id} p\left(Y_{2}\right) \Rightarrow \\
\mathrm{d} \pi\left(X_{1}\right)+\mathrm{i} d \pi\left(Y_{1}\right)=\mathrm{d} \pi\left(X_{2}\right)+\mathrm{id} \pi\left(Y_{2}\right) \Rightarrow \mathrm{d} \pi\left(X_{1}+I\left(Y_{1}\right)\right)=\mathrm{d} \pi\left(X_{2}+I\left(Y_{2}\right)\right) .
\end{gathered}
$$

The last equation implies $X_{1}+I\left(Y_{1}\right)=X_{2}+I\left(Y_{2}\right)$ (taking into account that $X_{1}+I\left(Y_{1}\right) \in \mathfrak{p}_{u}$ and $\left.X_{2}+I\left(Y_{2}\right) \in \mathfrak{p}_{u}\right)$, hence

$$
X_{1}-X_{2}=I\left(Y_{2}-Y_{1}\right) \Rightarrow I\left(X_{1}-X_{2}\right)=Y_{1}-Y_{2} .
$$

Furthermore, we have $\mathrm{i}\left(Y_{2}-Y_{1}\right)=X_{1}-X_{2}$ (see (70) $)$, therefore $\mathrm{i}\left(Y_{1}-Y_{2}\right) \in \mathfrak{p}_{u}$, besides

$$
\mathrm{d} \pi\left(\mathrm{i}\left(Y_{1}-Y_{2}\right)\right)=\mathrm{d} p\left(\mathrm{i}\left(Y_{1}-Y_{2}\right)\right)=\mathrm{id} p\left(Y_{1}-Y_{2}\right)=\mathrm{id} \pi\left(Y_{1}-Y_{2}\right)=\mathrm{d} \pi\left(I\left(Y_{1}-Y_{2}\right)\right),
$$

which implies

$$
\mathrm{i}\left(Y_{1}-Y_{2}\right)=I\left(Y_{1}-Y_{2}\right) \Rightarrow Y_{1}-Y_{2}+\mathrm{i} I\left(Y_{1}-Y_{2}\right)=0 .
$$

From (71) it follows that

$$
I\left(X_{1}-X_{2}\right)+\mathrm{i} I\left(Y_{1}-Y_{2}\right)=Y_{1}-Y_{2}+\mathrm{i} I\left(Y_{1}-Y_{2}\right)
$$

and now from (72) we infer

$$
I\left(X_{1}-X_{2}\right)+\mathrm{i} I\left(Y_{1}-Y_{2}\right)=0 \quad \Rightarrow \quad I\left(X_{1}\right)+\mathrm{i} I\left(Y_{1}\right)=I\left(X_{2}\right)+\mathrm{i} I\left(Y_{2}\right) .
$$

Hence we see that the operator on $\mathfrak{p}$ defined by the rule

$$
X+\mathrm{i} Y \mapsto I(X)+\mathrm{i} I(Y) \quad X, Y \in \mathfrak{p}_{u}
$$

is well defined and this is the extension of $I$ from $\mathfrak{p}_{u}$ to $\mathfrak{p}$.

Let $\mathfrak{p}^{+}$and $\mathfrak{p}^{-}$be the $\mathrm{i}$ and $(-\mathrm{i})$-eigenspaces of $I$, respectively, i. e.

$$
\mathfrak{p}^{+}=\{X \in \mathfrak{p} \mid I(X)=\mathrm{i} X\} \quad \mathfrak{p}^{-}=\{X \in \mathfrak{p} \mid I(X)=-\mathrm{i} X\} .
$$

In addition to (61) we have

$$
\forall X \in \mathfrak{p} \quad \mathrm{d} p(I(X))=\operatorname{id} p(X) .
$$

Indeed, let $X_{1}, X_{2} \in \mathfrak{p}_{u}$ then

$$
\begin{gathered}
\mathrm{d} p\left(I\left(X_{1}+\mathrm{i} X_{2}\right)\right)=\mathrm{d} p\left(I\left(X_{1}\right)+\mathrm{i} I\left(X_{2}\right)\right)=\mathrm{d} \pi\left(I\left(X_{1}\right)\right)+\mathrm{id} \pi\left(I\left(X_{2}\right)\right) \\
=\mathrm{id} \pi\left(X_{1}\right)+\operatorname{iid} \pi\left(X_{2}\right)=\mathrm{i}\left(\mathrm{d} \pi\left(X_{1}\right)+\mathrm{id} \pi\left(X_{2}\right)\right)=\mathrm{i}\left(\mathrm{d} p\left(X_{1}\right)+\mathrm{d} p\left(\mathrm{i} X_{2}\right)\right) \\
=\operatorname{id} p\left(X_{1}+\mathrm{i} X_{2}\right) .
\end{gathered}
$$


Lemma 4.4. The following equality is valid

$$
\mathfrak{l} \cap \mathfrak{p}=\mathfrak{p}^{-} . \quad \mathfrak{g}_{I}=\mathfrak{l} \oplus \mathfrak{p}^{+} .
$$

Proof. Let $Z=X+\mathrm{i} Y \in \mathfrak{l} \cap \mathfrak{p}$, where $X, Y \in \mathfrak{p}_{u}$, then we have

$$
0=\mathrm{d} p(X+\mathrm{i} Y)=\mathrm{d} p(X)+\mathrm{id} p(Y)=\mathrm{d} \pi(X)+\mathrm{d} \pi(I(Y))=\mathrm{d} \pi(X+I(Y)) .
$$

This equality, and $X+I(Y) \in \mathfrak{p}_{u}$, imply $X+I(Y)=0$. Therefore $Y=I(X)$ and $Z=$ $X+\mathrm{i} Y=X+\mathrm{i} I(X)$, hence $I(Z)=I(X+\mathrm{i} I(X))=-\mathrm{i}(X+\mathrm{i} I(X))=-\mathrm{i} Z$. Thus we proved that $\mathfrak{l} \cap \mathfrak{p} \subset \mathfrak{p}^{-}$. Conversely, let $Z \in \mathfrak{p}^{-}$, then $I(Z)=-\mathrm{i} Z$ and $Z=\mathrm{i} I(Z)$, therefore

$$
\mathrm{d} p(Z)=\mathrm{d} p(\mathrm{i} I(Z))=\operatorname{iid} p(Z)=-\mathrm{d} p(Z) \Rightarrow \mathrm{d} p(Z)=0,
$$

hence $Z \in \mathfrak{l}$ and we see that $\mathfrak{p}^{-} \subset \mathfrak{l} \cap \mathfrak{p}$. So we proved the first equality. Now we have $\mathfrak{l} \cap \mathfrak{p}^{+} \subset(\mathfrak{l} \cap \mathfrak{p}) \cap \mathfrak{p}^{+}=\mathfrak{p}^{-} \cap \mathfrak{p}^{+}=\{0\}$ and we see that $\mathfrak{l} \cap \mathfrak{p}^{+}=\{0\}$. Since $\mathfrak{u}=\mathfrak{k}_{u}+\mathfrak{p}_{u}$, then obviously $\mathfrak{g}_{I}=\mathfrak{k}+\mathfrak{p}$, hence $\mathfrak{g}_{I}=\mathfrak{k}+\mathfrak{p}^{+}+\mathfrak{p}^{-} \subset \mathfrak{l}+\mathfrak{p}^{+}$. The Lemma follows.

Corollary 4.5. The complex subspace $\mathfrak{p} \subset \mathfrak{g}_{I}$ is a complexification of $\mathfrak{p}_{u} \subset \mathfrak{u}$, $i$. e. we have

$$
\mathfrak{p}=\mathfrak{p}_{u} \oplus \mathfrak{i p}_{u} \quad \text { direct vector space sum. }
$$

In particular the complex structure $J$ on $\mathfrak{p}_{u}$ may be extended by complex linearity to the complex subspace $\mathfrak{p}$, we shall denote this extension by the same letter $J$.

Proof. By the previous lemma we have $\operatorname{dim}_{\mathbb{C}}\left(\mathfrak{p}^{+}\right)=\operatorname{dim}_{\mathbb{C}}\left(\mathfrak{g}_{I}\right)-\operatorname{dim}_{\mathbb{C}}(\mathfrak{l})=\operatorname{dim}_{\mathbb{C}}(M)$, hence $\operatorname{dim}_{\mathbb{C}}(\mathfrak{p})=2 \operatorname{dim}_{\mathbb{C}}\left(\mathfrak{p}^{+}\right)=\operatorname{dim}_{\mathbb{R}}(M)=\operatorname{dim}_{\mathbb{R}}\left(\mathfrak{p}_{u}\right)$. So, we see that

$$
\operatorname{dim}_{\mathbb{C}}(\mathfrak{p})=\operatorname{dim}_{\mathbb{R}}\left(\mathfrak{p}_{u}\right) \Rightarrow \operatorname{dim}_{\mathbb{R}}(\mathfrak{p})=2 \operatorname{dim}_{\mathbb{R}}\left(\mathfrak{p}_{u}\right) .
$$

On the other hand

$$
\operatorname{dim}_{\mathbb{R}}(\mathfrak{p})=\operatorname{dim}_{\mathbb{R}}\left(\mathfrak{p}_{u}\right)+\operatorname{dim}_{\mathbb{R}}\left(\mathfrak{i p}_{u}\right)-\operatorname{dim}_{\mathbb{R}}\left(\mathfrak{p}_{u} \cap \mathfrak{i p}_{u}\right)=2 \operatorname{dim}_{\mathbb{R}}\left(\mathfrak{p}_{u}\right)-\operatorname{dim}_{\mathbb{R}}\left(\mathfrak{p}_{u} \cap \mathfrak{i} \mathfrak{p}_{u}\right)
$$

and we infer (using $\left.\operatorname{dim}_{\mathbb{R}}(\mathfrak{p})=2 \operatorname{dim}_{\mathbb{R}}\left(\mathfrak{p}_{u}\right)\right)$ that $\operatorname{dim}_{\mathbb{R}}\left(\mathfrak{p}_{u} \cap \mathfrak{i p}_{u}\right)=0$, i. e. $\mathfrak{p}_{u} \cap \mathfrak{i p}_{u}=\{0\}$ and the corollary follows.

Remark 4.6. From Corollary 3.11 it follows that the subspace of the complexification $\mathfrak{g}$, generated by $\mathfrak{p}_{u}$, is isomorhic to the subspace $\mathfrak{p}$ of $\mathfrak{g}_{I}$. We shall identify these two subspaces and shall denote them by a common notation $\mathfrak{p}$ (the isomorphism, that identifies them maps a sum $X+\mathrm{i} Y$ in $\mathfrak{g}_{I}$, where $X, Y \in \mathfrak{p}_{u}$, to the sum $X+\mathrm{i} Y$ in $\left.\mathfrak{g}\right)$. Of course the complex structures $I, J$ and the subspaces $\mathfrak{p}^{+}, \mathfrak{p}^{-}$can be regarded as objects related to the subspace of $\mathfrak{g}$ generated by $\mathfrak{p}_{u}$ so, they satisfy the relations

$$
I \circ \tau=\tau \circ I \quad J \circ \tau=\tau \circ J \quad I \circ J=-J \circ I
$$

$$
\mathfrak{p}^{-}=\tau\left(\mathfrak{p}^{+}\right) \quad J\left(\mathfrak{p}^{+}\right)=\mathfrak{p}^{-} .
$$

Since $\mathfrak{g}$ is the complexification of $\mathfrak{u}$, the relations $\mathfrak{u}=\mathfrak{k}_{u}+\mathfrak{p}_{u}, \mathfrak{k}_{u} \cap \mathfrak{p}_{u}=\{0\},\left[\mathfrak{k}_{u}, \mathfrak{p}_{u}\right] \subset \mathfrak{p}_{u}$ imply

$$
\mathfrak{g}=\mathfrak{k} \oplus \mathfrak{p}, \quad[\mathfrak{k}, \mathfrak{p}] \subset \mathfrak{p} .
$$


4.4. Fixing the Cartan subalgebra. The coset space $\mathbf{G}_{I} / \mathbf{L}$ is compact. From the normalizer theorem (A. Borel - R. Remmert) (see e.g. [1] (page 80)), it follows that the normalizer $\mathfrak{n}(\mathfrak{l})$ of $\mathfrak{l}$ in $\mathfrak{g}_{I}$ is a parabolic subalgebra of $\mathfrak{g}_{I}$, i. e.

$$
\mathfrak{n}(\mathfrak{l})=\mathfrak{c}_{I} \oplus \mathfrak{s}
$$

where $\mathfrak{s}$ is a parabolic subalgebra of $\mathfrak{g}_{s}$ and $\mathfrak{c}_{I}$ is the center of $\mathfrak{g}_{I}$ (see (58)). Let us recall that $\tau_{s}$ is the conjugation of $\mathfrak{g}_{s}$, which corresponds to the compact real form $\mathfrak{u}_{s} \subset \mathfrak{g}_{s}$. Since $\mathfrak{s}$ is a parabolic subalgebra of $\mathfrak{g}_{s}$ then (see [19], Theorem 2.6 ) there exists a $\tau_{s}$-invariant Cartan subalgebra $\mathfrak{h}_{s}$ of $\mathfrak{g}_{s}$, such that $\mathfrak{h}_{s} \subset \mathfrak{s}$. Let us denote (recall that we identify the semisimple part of $\mathfrak{g}$ with the semisimple part of $\mathfrak{g}_{I}$ and denote them by the common notation $\mathfrak{g}_{s}$ ):

$$
\mathfrak{h}_{I}=\mathfrak{c}_{I} \oplus \mathfrak{h}_{s} \subset \mathfrak{g}_{I}, \quad \mathfrak{h}=\mathfrak{c} \oplus \mathfrak{h}_{s} \subset \mathfrak{g}
$$

then $\mathfrak{h}_{I}$ and $\mathfrak{h}$ are Cartan subalgebras of the reductive algebras $\mathfrak{g}_{I}$ and $\mathfrak{g}$, respectively, and we obtain that $\mathfrak{h}_{I} \subset \mathfrak{n}(\mathfrak{l})$, i. e.

$$
\left[\mathfrak{h}_{I}, \mathfrak{l}\right] \subset \mathfrak{l} .
$$

We racall that $\tau_{\mid \mathfrak{g}_{s}}=\tau_{s}, \tau(\mathfrak{c})=\mathfrak{c}($ see $(67))$, hence

$$
\tau(\mathfrak{h})=\mathfrak{h} .
$$

Remark 4.7. Further in this text, we treat the algebra $\mathfrak{g}$ and its Cartan subalgebra $\mathfrak{h}$, the algebras $\mathfrak{g}_{I}$ and $\mathfrak{h}_{I} \subset \mathfrak{g}_{I}$ are used only for the proof of the regularity of $\mathfrak{k}$ and $\mathfrak{p}$.

So everywhere $\Delta$ is the root system w.r. to the Cartan subalgebra $\mathfrak{h} \subset \mathfrak{g}$ and we fix a Weyl-Chevalley basis of $\mathfrak{g}$ as in (2).

The choice of the positive roots $\Delta^{+} \subset \Delta$ will be explained in subsection 4.6 .

4.5. Regularity of $\mathfrak{k}$ and $\mathfrak{p}$. We have shown that $\mathfrak{l}$ is a regular subalgebra of $\mathfrak{g}_{I}$. In this subsection we shall prove that $\mathfrak{k}$ and $\mathfrak{p}$ are regular in $\mathfrak{g}$ (for the notations $\mathfrak{k}$ and $\mathfrak{p}$ see (66) and Remark 4.6).

Let us recall first that $\mathfrak{h}_{s}$ is $\tau_{s}$-invariant and therefore we have

$$
\mathfrak{h}_{s}=\left(\mathfrak{h}_{s} \cap \mathfrak{u}_{s}\right) \oplus \mathrm{i}\left(\mathfrak{h}_{s} \cap \mathfrak{u}_{s}\right),
$$

for the definition of $\mathfrak{u}_{s}$ see (11) and Lemma 4.1.

Lemma 4.8. The subalgebra $\mathfrak{k} \subset \mathfrak{g}$ is regular $w$. r. to $\mathfrak{h}, i$. e.

$$
\mathfrak{k}=(\mathfrak{k} \cap \mathfrak{h}) \oplus \bigoplus_{\alpha \in \Delta_{\mathfrak{k}}} \mathfrak{g}(\alpha), \quad \text { where } \quad \Delta_{\mathfrak{k}}=\{\alpha \in \Delta \mid \mathfrak{g}(\alpha) \subset \mathfrak{k}\}
$$

The set $\Delta_{\mathfrak{k}}$ is symmetric with respect to $0, i$. e. $-\Delta_{\mathfrak{k}}=\Delta_{\mathfrak{k}}$.

Consequently $\mathfrak{p}$ is a regular subspace of $\mathfrak{g}$.

Proof. Since $\left[\mathfrak{h}_{I}, \mathfrak{l}\right] \subset \mathfrak{l}$ then $\left[\mathfrak{h}_{s} \cap \mathfrak{u}_{s}, \mathfrak{l}\right] \subset \mathfrak{l}$, besides since $\mathfrak{u}$ is a subalgebra then $\left[\mathfrak{h}_{s} \cap \mathfrak{u}_{s}, \mathfrak{u}\right] \subset \mathfrak{u}$, hence $\left[\mathfrak{h}_{s} \cap \mathfrak{u}_{s}, \mathfrak{u} \cap \mathfrak{l}\right] \subset \mathfrak{u} \cap \mathfrak{l}$. On the other hand $\mathfrak{k}_{u}=\mathfrak{l} \cap \mathfrak{u}$ (see (62)), which implies

$$
\left[\mathfrak{h}_{s} \cap \mathfrak{u}_{s}, \mathfrak{k}_{u}\right] \subset \mathfrak{k}_{u},
$$


which, by (81) and $\mathfrak{k}=\mathfrak{k}_{u}+\mathfrak{i k}_{u} \subset \mathfrak{g}$, implies $\left[\mathfrak{h}_{s}, \mathfrak{k}\right] \subset \mathfrak{k}$. Now (178) gives $[\mathfrak{h}, \mathfrak{k}] \subset \mathfrak{k}$, i. e. $\mathfrak{k}$ is regular and we obtain (82).

The set $\Delta_{\mathfrak{k}}$ is symmetric with respect to 0 , i. e. $-\Delta_{\mathfrak{k}}=\Delta_{\mathfrak{k}}$, since $\tau(\mathfrak{k})=\mathfrak{k}$ and $\tau(\mathfrak{g}(\alpha))=$ $\mathfrak{g}(-\alpha)$ for $\alpha \in \Delta$.

Since $\mathfrak{p}_{u}$ is the orthogonal complement of $\mathfrak{k}_{u}$ in $\mathfrak{u}$ with respect to the Killing form, hence the regularity of $\mathfrak{p}$ follows from the regularity of $\mathfrak{k}$.

Now we shall prove the regularity of $\mathfrak{p}^{+}$and $\mathfrak{p}^{-}$

Lemma 4.9. The subspaces $\mathfrak{p}^{+}$and $\mathfrak{p}^{-}$are regular in $\mathfrak{g} w$. r. to $\mathfrak{h}, i$. e.

$$
\begin{aligned}
& \mathfrak{p}^{+}=\left(\mathfrak{p}^{+} \cap \mathfrak{h}\right) \oplus \bigoplus_{\alpha \in \Delta_{\mathfrak{p}}^{+}} \mathfrak{g}(\alpha), \text { where } \Delta_{\mathfrak{p}}^{+}=\left\{\alpha \mid \mathfrak{g}(\alpha) \subset \mathfrak{p}^{+}\right\} \\
& \mathfrak{p}^{-}=\left(\mathfrak{h} \cap \mathfrak{p}^{-}\right) \oplus \bigoplus_{\alpha \in \Delta_{\mathfrak{p}}^{+}} \mathfrak{g}(-\alpha) \quad \mathfrak{h} \cap \mathfrak{p}^{-}=\tau\left(\mathfrak{h} \cap \mathfrak{p}^{+}\right) .
\end{aligned}
$$

Furthermore,

$$
\begin{array}{rr}
\mathfrak{h} \cap \mathfrak{p}=\left(\mathfrak{h} \cap \mathfrak{p}^{+}\right) \oplus\left(\mathfrak{h} \cap \mathfrak{p}^{-}\right) & \mathfrak{h}=(\mathfrak{h} \cap \mathfrak{p}) \oplus(\mathfrak{h} \cap \mathfrak{k}) \\
\Delta \backslash \Delta_{\mathfrak{k}}=\Delta_{\mathfrak{p}}^{+} \cup\left(-\Delta_{\mathfrak{p}}^{+}\right) & \Delta_{\mathfrak{p}}^{+} \cap\left(-\Delta_{\mathfrak{p}}^{+}\right)=\emptyset .
\end{array}
$$

Proof. We have proved that $\mathfrak{l}$ and $\mathfrak{p}$ are regular subalgebras of $\mathfrak{g}_{I}$ with respect to the Cartan subalgebra $\mathfrak{h}_{I}$, i. e. $\left[\mathfrak{h}_{I}, \mathfrak{l}\right] \subset \mathfrak{l}$ and $\left[\mathfrak{h}_{I}, \mathfrak{p}\right] \subset \mathfrak{p}$. Therefore $\left[\mathfrak{h}_{I}, \mathfrak{l} \cap \mathfrak{p}\right] \subset \mathfrak{l} \cap \mathfrak{p}$. On the other hand $\mathfrak{l} \cap \mathfrak{p}=\mathfrak{p}^{-}$(see Lemma 4.4). Therefore $\left[\mathfrak{h}_{I}, \mathfrak{p}^{-}\right] \subset \mathfrak{p}^{-}$, i. e. $\mathfrak{p}^{-}$is regular in $\mathfrak{g}_{I}$ with respect to $\mathfrak{h}_{I}$. Now obviously $\left[\mathfrak{h}_{u}, \mathfrak{p}^{-}\right] \subset \mathfrak{p}^{-}$in $\mathfrak{g}_{I}$, hence one can verify, taking into account $\left[\mathfrak{h}_{u}, \mathfrak{p}_{u}\right] \subset \mathfrak{p}_{u}$ and Remark 4.6, that $\left[\mathfrak{h}_{u}, \mathfrak{p}^{-}\right] \subset \mathfrak{p}^{-}$in $\mathfrak{g}$ as well. Therefore $\left[\mathfrak{h}, \mathfrak{p}^{-}\right] \subset \mathfrak{p}^{-}$in $\mathfrak{g}$ and $\mathfrak{p}^{-}$is regular. From (75) in Remark 4.6 we know that $\mathfrak{p}^{+}=\tau\left(\mathfrak{p}^{-}\right)$, besides $\mathfrak{h}$ is $\tau$-invariant, therefore $\mathfrak{p}^{+}$is regular as well, so we obtain (84)).

From (84) and $\tau(\mathfrak{g}(\alpha))=\mathfrak{g}(-\alpha)$, it follows that

$$
\mathfrak{p}^{-}=\tau\left(\mathfrak{p}^{+} \cap \mathfrak{h}\right)+\sum_{\alpha \in \Delta_{\mathfrak{p}}^{+}} \mathfrak{g}(-\alpha) .
$$

We see that $\mathfrak{p}^{-}$is regular in $\mathfrak{g}$ and $\mathfrak{h} \cap \mathfrak{p}^{-}=\tau\left(\mathfrak{h} \cap \mathfrak{p}^{+}\right)$, therefore we have proved (85).

Since $\mathfrak{p}=\mathfrak{p}^{+}+\mathfrak{p}^{-}, \mathfrak{p}^{+} \cap \mathfrak{p}^{-}=\{0\}$, then

$$
\Delta_{\mathfrak{p}}^{+} \cap\left(-\Delta_{\mathfrak{p}}^{+}\right)=\{0\} \quad \mathfrak{h} \cap \mathfrak{p}=\left(\mathfrak{h} \cap \mathfrak{p}^{+}\right) \oplus\left(\mathfrak{h} \cap \mathfrak{p}^{-}\right) .
$$

Since we have a direct vector sum $\mathfrak{g}=\mathfrak{k}+\mathfrak{p}^{+}+\mathfrak{p}^{-}$and Lemma (4.8) then obviously $\Delta \backslash \Delta_{\mathfrak{k}}=$ $\Delta_{\mathfrak{p}}^{+} \cup\left(-\Delta_{\mathfrak{p}}^{+}\right)$.

4.6. The set $\Delta_{\mathfrak{p}}^{+}$. We recall that the definitions of $I$ and $J$ are in (69). Integrability of the complex structure $I_{M}$ on $M$ implies (see [10], ch. X) that for $X, Y \in \mathfrak{p}_{u}$ we have

$$
[I X, I Y]_{\mathfrak{p}_{u}}-[X, Y]_{\mathfrak{p}_{u}}-I\left([X, I Y]_{\mathfrak{p}_{u}}\right)-I\left([I X, Y]_{\mathfrak{p}_{u}}\right)=0 .
$$

After complexification we can write

$$
\forall X, Y \in \mathfrak{p} \quad[I X, I Y]_{\mathfrak{p}}-[X, Y]_{\mathfrak{p}}-I\left([X, I Y]_{\mathfrak{p}}\right)-I\left([I X, Y]_{\mathfrak{p}}\right)=0 .
$$


The integrability of I implies

$$
\forall X, Y \in \mathfrak{p}^{ \pm} \quad[X, Y]_{\mathfrak{p}} \in \mathfrak{p}^{ \pm} .
$$

The elements in $\mathbf{U}$ act as hypercomplex diffeomorphisms of $M$, whence for $X \in \mathfrak{k}$ we have

$$
I \circ \operatorname{ad}(X)=\operatorname{ad}(X) \circ I, \quad J \circ \operatorname{ad}(X)=\operatorname{ad}(X) \circ J .
$$

We recall that (76) is valid, therefore the equations above make sense. Relation (89) implies

$$
\left[\mathfrak{k}, \mathfrak{p}^{+}\right] \subset \mathfrak{p}^{+}, \quad\left[\mathfrak{k}, \mathfrak{p}^{-}\right] \subset \mathfrak{p}^{-} .
$$

Indeed, let $X \in \mathfrak{k}, Y \in \mathfrak{p}^{+}$then

$$
[X, Y]=-\mathrm{i} a d(X)(I(Y))=-\mathrm{i} I(\operatorname{ad}(X)(Y))=-\mathrm{i} I([X, Y]) \Rightarrow I([X, Y])=\mathrm{i}[X, Y]
$$

hence $[X, Y] \in \mathfrak{p}^{+}$. The proof for $\mathfrak{p}^{-}$is the same.

From (90), (88) and since $\mathfrak{k}$ is a subalgebra of $\mathfrak{g}$ it follows that $\mathfrak{k}+\mathfrak{p}^{+}$is a subalgebra of $\mathfrak{g}$. Furthermore, both $\mathfrak{k}$ and $\mathfrak{p}^{+}$are regular, therefore $\mathfrak{k}+\mathfrak{p}^{+}$is regular and obviously by (84) and (82) we have

$$
\mathfrak{k}+\mathfrak{p}^{+}=\left(\mathfrak{k}+\mathfrak{p}^{+}\right) \cap \mathfrak{h}+\sum_{\alpha \in \Delta_{\mathfrak{k}} \cup \Delta_{\mathfrak{p}}^{+}} \mathfrak{g}(\alpha) .
$$

From (87) we see, that $\Delta=\Delta_{\mathfrak{k}} \cup \Delta_{\mathfrak{p}}^{+} \cup\left(-\Delta_{\mathfrak{p}}^{+}\right)=\left(\Delta_{\mathfrak{k}} \cup \Delta_{\mathfrak{p}}^{+}\right) \cup\left(-\left(\Delta_{\mathfrak{k}} \cup \Delta_{\mathfrak{p}}^{+}\right)\right)$. From ([5], ChVII, §3, n.4) we see that (91), $\Delta=\left(\Delta_{\mathfrak{k}} \cup \Delta_{\mathfrak{p}}^{+}\right) \cup\left(-\left(\Delta_{\mathfrak{k}} \cup \Delta_{\mathfrak{p}}^{+}\right)\right)$and the fact that $\mathfrak{k}+\mathfrak{p}^{+}$is a subalgebra imply that there exists a basis $\Pi$ of the root system $\Delta$, such that the set of positive roots $\Delta^{+}$, for this basis $\Pi$, satisfies $\Delta^{+} \subset \Delta_{\mathfrak{k}} \cup \Delta_{\mathfrak{p}}^{+}$. Since $\Delta^{+} \cup\left(-\Delta^{+}\right)=\Delta$, then for any $\alpha \in \Delta_{\mathfrak{p}}^{+}$we have $\alpha \in \Delta^{+}$or $-\alpha \in \Delta^{+}$, but the relation $-\alpha \in \Delta^{+}$contradicts $\Delta^{+} \subset \Delta_{\mathfrak{k}} \cup \Delta_{\mathfrak{p}}^{+}$ and (87). Thus we proved the existence of such a basis $\Pi$, that the corresponding set of positive roots satisfies

$$
\Delta_{\mathfrak{p}}^{+} \subset \Delta^{+}
$$

Remark 4.10. From now on this subset of positive roots $\Delta^{+}$will be fixed throughout section 4. It is useful to denote also

$$
\Delta_{\mathfrak{k}}^{+}=\Delta^{+} \cap \Delta_{\mathfrak{k}}
$$

Then from (87) it follows

$$
\Delta^{+}=\Delta_{\mathfrak{k}}^{+} \cup \Delta_{\mathfrak{p}}^{+} \quad \text { disjoint union } .
$$

In the sequel we shall use also the following notations, related to the stem $(\Gamma, \prec)$ of $\Delta^{+}$:

$$
\begin{gathered}
\Phi_{\zeta}^{\mathfrak{p}}=\left\{\beta \in \Delta_{\mathfrak{p}}^{+} \mid \zeta-\beta \in \Delta_{\mathfrak{p}}^{+}\right\} \quad \zeta \in \Delta_{\mathfrak{p}}^{+} \\
\Gamma_{\mathfrak{p}}=\Gamma \cap \Delta_{\mathfrak{p}}^{+}, \quad \Gamma_{\mathfrak{k}}=\Gamma \cap \Delta_{\mathfrak{k}}^{+}=\Gamma \backslash \Gamma_{\mathfrak{p}}, \quad \mathfrak{w}_{\mathfrak{p}}=\operatorname{span}_{\mathbb{R}}\left\{W_{\gamma} \mid \gamma \in \Gamma_{\mathfrak{p}}\right\} .
\end{gathered}
$$


4.7. Decomposition of the Cartan subalgebra: $\mathfrak{h}_{u}, \mathfrak{h}_{\mathfrak{k}_{u}}, \mathfrak{h}_{\mathfrak{p}_{u}}, \mathfrak{n}_{\mathfrak{p}_{u}}$. We study the structure of $\Delta_{\mathfrak{k}}^{+}, \Delta_{\mathfrak{p}}^{+}$to show that $\mathfrak{k}$ is a $\Gamma_{\mathfrak{k}}$-stemmed subalgebra. We also show that $J$ is of the type (49). First we introduce some more notations. Let us denote

$$
\mathfrak{h}_{u}=\mathfrak{u} \cap \mathfrak{h}=\mathfrak{c}_{u} \oplus\left(\mathfrak{h}_{s} \cap \mathfrak{u}_{s}\right), \quad \mathfrak{h}_{\mathfrak{k}_{u}}=\mathfrak{k}_{u} \cap \mathfrak{h}, \quad \mathfrak{h}_{\mathfrak{p}_{u}}=\mathfrak{p}_{u} \cap \mathfrak{h} .
$$

Since $\mathfrak{u}=\mathfrak{k}_{u} \oplus \mathfrak{p}_{u}$, then obviously

$$
\mathfrak{h}_{u}=\mathfrak{h}_{\mathfrak{k}_{u}} \oplus \mathfrak{h}_{\mathfrak{p}_{u}}
$$

Furthermore, let us denote

$$
\begin{gathered}
\mathfrak{n}_{\mathfrak{p}_{u}}=\operatorname{span}_{\mathbb{R}}\left\{\left(E_{\alpha}-E_{-\alpha}\right), \mathrm{i}\left(E_{\alpha}+E_{-\alpha}\right) ; \alpha \in \Delta_{\mathfrak{p}}\right\} \\
\mathfrak{n}_{\mathfrak{p}}^{+}=\sum_{\alpha \in \Delta_{\mathfrak{p}}^{+}} \mathfrak{g}(\alpha) \quad \mathfrak{n}_{\mathfrak{p}}^{-}=\sum_{\alpha \in \Delta_{\mathfrak{p}}^{+}} \mathfrak{g}(-\alpha) \quad \mathfrak{n}_{\mathfrak{p}}=\mathfrak{n}_{\mathfrak{p}}^{+}+\mathfrak{n}_{\mathfrak{p}}^{-}
\end{gathered}
$$

then obviously

$$
\mathfrak{p}_{u}=\mathfrak{h}_{\mathfrak{p}_{u}} \oplus \mathfrak{n}_{\mathfrak{p}_{u}}, \quad \mathfrak{k}_{u}=\mathfrak{h}_{\mathfrak{k}_{u}} \oplus \operatorname{span}_{\mathbb{R}}\left\{\left(E_{\alpha}-E_{-\alpha}\right), \mathrm{i}\left(E_{\alpha}+E_{-\alpha}\right) ; \alpha \in \Delta_{\mathfrak{k}}\right\}
$$

With these notations the following lemmas are valid

Lemma 4.11. If $\alpha \in \Delta$ and $\alpha\left(\mathfrak{h}_{\mathfrak{k}_{u}}\right)=0$, then $W_{\alpha} \in \mathfrak{p}_{u}$ (recall that $\left.W_{\alpha}=\frac{i}{2} H_{\alpha}\right)$.

Proof. Let $X \in \mathfrak{h}_{\mathfrak{k}_{u}}$. Let us decompose $X=X_{\mathfrak{c}}+X_{s}, X_{\mathfrak{c}} \in \mathfrak{c}_{u}, X_{s} \in \mathfrak{u}_{s} \cap \mathfrak{h}_{s}$ in accordance with the decomposition $\mathfrak{h}_{u}=\mathfrak{c}_{u} \oplus\left(\mathfrak{h}_{s} \cap \mathfrak{u}_{s}\right)$. Since $\alpha(X)=0$ and $\alpha$ vanishes on $\mathfrak{c}_{u}$, then $\alpha\left(X_{s}\right)=0$. Hence $\operatorname{Kill}\left(h_{\alpha}, X_{s}\right)=\alpha\left(X_{s}\right)=0$ and $\left\langle W_{\alpha}, X\right\rangle=\left\langle W_{\alpha}, X_{\mathfrak{c}}+X_{s}\right\rangle=\left\langle W_{\alpha}, X_{s}\right\rangle=$ $\operatorname{Kill}\left(W_{\alpha}, X_{s}\right)=0$.

Using (101) and the obvious fact that $\left\langle W_{\alpha}, \mathfrak{n}^{ \pm}\right\rangle=0$ we see that $\left\langle\mathfrak{k}_{\mathfrak{u}}, W_{\alpha}\right\rangle=0$, hence $W_{\alpha} \in \mathfrak{p}_{u}$.

Lemma 4.12. Let $X \in \mathfrak{h}_{\mathfrak{p}_{u}}$ and $\alpha \in \Delta_{\mathfrak{k}}$ then $\alpha(X)=0$.

Proof. Since $E_{\alpha} \in \mathfrak{k}$ and $-\Delta_{\mathfrak{k}}=\Delta_{\mathfrak{k}}$ then $H_{\alpha}=\left[E_{\alpha}, E_{-\alpha}\right] \in \mathfrak{k} \cap \mathfrak{h}$. Therefore $\mathrm{i} H_{\alpha} \in \mathfrak{h}_{\mathfrak{k}_{u}}$. From the definition of $\mathfrak{p}_{u}$ we have $\left\langle\mathrm{i} H_{\alpha}, X\right\rangle=0$. Let us decompose $X=X_{\mathfrak{c}}+X_{s}, X_{\mathfrak{c}} \in \mathfrak{c}_{u}$, $X_{s} \in \mathfrak{u}_{s} \cap \mathfrak{h}_{s}$. Since $\alpha$ vanishes on $\mathfrak{c}_{u} \subset \mathfrak{c}$, then $\alpha\left(X_{s}\right)=\alpha(X)$. Since $\left\langle\mathrm{i} H_{\alpha}, X\right\rangle=0$ then $\left\langle\mathrm{i} H_{\alpha}, X_{s}\right\rangle=\alpha\left(X_{s}\right)=0$, therefore $\alpha(X)=0$.

4.8. Preliminary lemmas about $\mathfrak{k}, \mathfrak{p}^{+}, \mathfrak{p}^{-}$. Here we prove that $\Gamma_{\mathfrak{k}}$ is a substem of $(\Gamma, \prec)$.

Lemma 4.13. If $\gamma \in \Gamma_{\mathfrak{k}}$ and $\widetilde{\gamma} \succeq \gamma$, then $\Phi_{\widetilde{\gamma}} \cup\{\widetilde{\gamma},-\widetilde{\gamma}\} \subset \Delta_{\mathfrak{k}}$. In particular $\Phi_{\widetilde{\gamma}}^{+} \cup\{\widetilde{\gamma}\} \subset \Delta_{\mathfrak{k}}^{+}$.

Proof. Since $-\Delta_{\mathfrak{k}}=\Delta_{\mathfrak{k}}$ then $E_{-\gamma} \in \mathfrak{k}$. Let $\alpha \in \Phi_{\gamma}^{+}$. Let us assume that $\alpha \notin \Delta_{\mathfrak{k}}$. Then $\alpha \in \Delta^{+} \backslash \Delta_{\mathfrak{k}}$, therefore $\alpha \in \Delta_{\mathfrak{p}}^{+}$and $E_{\alpha} \in \mathfrak{p}^{+}$. By (90) $\left[E_{-\gamma}, E_{\alpha}\right] \in \mathfrak{p}^{+}$, but $\left[E_{-\gamma}, E_{\alpha}\right]=$ $N_{-\gamma, \alpha} E_{\alpha-\gamma}$ and $\alpha-\gamma \notin \Delta^{+}$(see (9)) , which contradicts $\Delta_{\mathfrak{p}}^{+} \subset \Delta^{+}$(see 92).

Thus, we see that if $\alpha \in \Phi_{\gamma} \cup\{-\gamma, \gamma\}$, then $E_{\alpha} \in \mathfrak{k}$. Now the lemma follows from (11) and the fact that $\mathfrak{k}$ is a subalgebra.

Corollary 4.14. The subset $\Gamma_{\mathfrak{k}} \subset \Gamma$ is a substem of $(\Gamma, \prec)$. 
Lemma 4.15. Let $\gamma \in \Gamma_{\mathfrak{p}}$ and $H_{\gamma} \in \mathfrak{k}$. Let

$$
J\left(E_{\gamma}\right)=H+\sum_{\alpha \in \Delta_{\mathfrak{p}}^{+}} c_{\alpha} E_{-\alpha} \quad H \in \mathfrak{h} \cap \mathfrak{p}^{-}, \quad c_{\alpha} \in \mathbb{C} .
$$

Then $H=0$ and

$$
\forall \alpha \in \Delta_{\mathfrak{p}}^{+} \quad \alpha\left(H_{\gamma}\right) \geq 0 \Rightarrow c_{\alpha}=0 .
$$

Proof. Since $H_{\gamma} \in \mathfrak{k}$ then by (89) it follows

$$
J\left(\left[H_{\gamma}, E_{\gamma}\right]\right)=\left[H_{\gamma}, J\left(E_{\gamma}\right)\right] \quad \Rightarrow \quad \gamma\left(H_{\gamma}\right) J\left(E_{\gamma}\right)=\sum_{\alpha \in \Delta_{\mathfrak{p}}^{+}} c_{\alpha}\left[H_{\gamma}, E_{-\alpha}\right]
$$

hence

$$
\gamma\left(H_{\gamma}\right)\left(H+\sum_{\alpha \in \Delta_{\mathfrak{p}}^{+}} c_{\alpha} E_{-\alpha}\right)=-\sum_{\alpha \in \Delta_{\mathfrak{p}}^{+}} c_{\alpha} \alpha\left(H_{\gamma}\right) E_{-\alpha}
$$

and we obtain

$$
\gamma\left(H_{\gamma}\right) H+\sum_{\alpha \in \Delta_{\mathfrak{p}}^{+}} c_{\alpha}(\gamma+\alpha)\left(H_{\gamma}\right) E_{-\alpha}=0 .
$$

Now we have $\gamma\left(H_{\gamma}\right)=2$ and therefore $(\gamma+\alpha)\left(H_{\gamma}\right) \neq 0$, if $\alpha\left(H_{\gamma}\right) \geq 0$, therefore the last equation above implies $H=0$ and (103).

Lemma 4.16. Let $\gamma \in \Gamma_{\mathfrak{p}}$ be a maximal root. Then $H_{\gamma} \notin \mathfrak{k}$.

Proof. Let us recall that $J\left(\mathfrak{p}^{+}\right)=\mathfrak{p}^{-}$, besides by $\gamma \in \Gamma_{\mathfrak{p}}$ we have $E_{\gamma} \in \mathfrak{p}^{+}$(see (196)) and (84)), therefore

$$
J\left(E_{\gamma}\right)=H+\sum_{\alpha \in \Delta_{\mathfrak{p}}^{+}} c_{\alpha} E_{-\alpha} \quad H \in \mathfrak{h} \cap \mathfrak{p}^{-}, c_{\alpha} \in \mathbb{C} .
$$

Let us assume that $H_{\gamma} \in \mathfrak{k}$. Now, since $\gamma$ is a maximal root, we have $\forall \alpha \in \Delta_{\mathfrak{p}}^{+}, \alpha\left(H_{\gamma}\right) \geq 0$, therefore Lemma 4.15 implies

$$
\left(H=0, \quad \forall \alpha \in \Delta_{\mathfrak{p}}^{+} \quad c_{\alpha}=0\right) \quad \Rightarrow \quad J\left(E_{\gamma}\right)=0
$$

which contradicts $J^{2}=-I d$.

Lemma 4.17. Let $\gamma \in \Delta_{\mathfrak{p}}^{+}$be such that $J\left(E_{\gamma}\right) \in \mathfrak{h}$, then $W_{\gamma} \in \mathfrak{p}_{u}$, i. e. $H_{\gamma} \in \mathfrak{p}$.

Proof. By (89) and $J\left(E_{\gamma}\right) \in \mathfrak{h}$ it follows that

$$
\forall H \in \mathfrak{h} \cap \mathfrak{k} \quad J\left(\left[H, E_{\gamma}\right]\right)=\left[H, J\left(E_{\gamma}\right)\right]=0 .
$$

Therefore $\forall H \in \mathfrak{h} \cap \mathfrak{k} \quad \gamma(H) J\left(E_{\gamma}\right)=0$, hence $\forall H \in \mathfrak{h} \cap \mathfrak{k} \quad \gamma(H)=0$. The lemma follows from Lemma 4.11.

Lemma 4.18. Let $\gamma \in \Gamma_{\mathfrak{p}}$ be such that $J\left(E_{\gamma}\right) \in \mathfrak{h}$, then $\Phi_{\gamma}^{+} \subset \Delta_{\mathfrak{p}}^{+}$. 
Proof. Let $\alpha \in \Phi_{\gamma}^{+}$. Let us assume that $\alpha \notin \Delta_{\mathfrak{p}}^{+}$. Then $\alpha \in \Delta_{\mathfrak{k}}^{+}$and $E_{\alpha}, E_{-\alpha} \in \mathfrak{k}$, besides $E_{\gamma} \in \mathfrak{p}^{+}\left(\right.$since $\left.\gamma \in \Gamma_{\mathfrak{p}}=\Gamma \cap \Delta_{\mathfrak{p}}^{+}\right)$. Since $\left[\mathfrak{k}, \mathfrak{p}^{+}\right] \subset \mathfrak{p}^{+}$, then (we recall that $\gamma-\alpha \in \Delta_{+}$)

$$
\left[E_{\gamma}, E_{-\alpha}\right]=N_{\gamma,-\alpha} E_{\gamma-\alpha} \in \mathfrak{p}^{+} \Rightarrow E_{\gamma-\alpha} \in \mathfrak{p}^{+} .
$$

There exist $H \in \mathfrak{h} \cap \mathfrak{p}^{-}$and constants $c_{\beta} \in \mathbb{C}$ for $\beta \in \Delta_{\mathfrak{p}}^{+}$, such that

$$
J\left(E_{\gamma-\alpha}\right)=H+\sum_{\beta \in \Delta_{\mathfrak{p}}^{+}} c_{\beta} E_{-\beta} .
$$

From (89) it follows

$$
\left[E_{\alpha}, J\left(E_{\gamma-\alpha}\right)\right]=J\left(\left[E_{\alpha}, E_{\gamma-\alpha}\right]\right) \Rightarrow\left[E_{\alpha}, J\left(E_{\gamma-\alpha}\right)\right]=N_{\alpha, \gamma-\alpha} J\left(E_{\gamma}\right) \in \mathfrak{h} \backslash\{0\}
$$

therefore

$$
\left[E_{\alpha}, H+\sum_{\beta \in \Delta_{\mathfrak{p}}^{+}} c_{\beta} E_{-\beta}\right]=-\alpha(H) E_{\alpha}+\sum_{\beta \in \Delta_{\mathfrak{p}}^{+}} c_{\beta}\left[E_{\alpha}, E_{-\beta}\right]=N_{\alpha, \gamma-\alpha} J\left(E_{\gamma}\right) \in \mathfrak{h} \backslash\{0\} .
$$

However, for $\beta \in \Delta_{\mathfrak{p}}^{+}$we have $\alpha \pm \beta \neq 0$ (since $\alpha \in \Delta_{\mathfrak{k}}$ ), therefore

$$
-\alpha(H) E_{\alpha}+\sum_{\beta \in \Delta_{\mathfrak{p}}^{+}} c_{\beta}\left[E_{\alpha}, E_{-\beta}\right] \notin \mathfrak{h} .
$$

This contradicts our assumption $\alpha \notin \Delta_{\mathfrak{p}}^{+}$whence the lemma follows.

4.9. The integrability condition. Throughout section 4 the notations $\mathfrak{p}_{u}, I, J, \mathfrak{p}^{+}, \mathfrak{p}^{-}$ have the same meaning (see Section 4.3) as well as $\mathfrak{h}_{\mathfrak{p}_{u}}$ (see (97)). We recall that $\mathfrak{k}_{u} \oplus \mathfrak{p}_{u}=\mathfrak{u}$ and that the reductivity condition $\left[\mathfrak{k}_{u}, \mathfrak{p}_{u}\right] \subset \mathfrak{p}_{u}$ is satisfied. In this section we shall vary $\mathfrak{h}_{\mathfrak{p}_{u}}$ to another $\mathfrak{q}$, with corresponding $\widetilde{\mathfrak{p}}_{u}=\mathfrak{q}+\mathfrak{n}_{\mathfrak{p}_{u}}$, observing the condition $\mathfrak{k}_{u} \oplus \widetilde{\mathfrak{p}}_{u}=\mathfrak{u}$, but we discard the reductivity condition.

4.9.1. Construction of $\left(\widetilde{\mathfrak{p}}, \widetilde{\mathfrak{p}}^{+}, \widetilde{J}, \widetilde{\xi}, \widetilde{\eta}, \widetilde{b}\right)$ from $\mathfrak{q}$. Let $\mathfrak{q}$ be any subspce of $\mathfrak{h}_{u}$, such that

$$
\mathfrak{h}_{u}=\mathfrak{q} \oplus \mathfrak{h}_{\mathfrak{k}_{u}}
$$

then we denote $\widetilde{\mathfrak{p}}_{u}=\mathfrak{q}+\mathfrak{n}_{\mathfrak{p}_{u}}$.

We denote by $\widetilde{\mathfrak{p}}$ the complexification of $\widetilde{\mathfrak{p}}_{u}$. Obviously

$$
\widetilde{\mathfrak{p}}=(\mathfrak{h} \cap \widetilde{\mathfrak{p}})+\mathfrak{n}_{\mathfrak{p}} .
$$

From (101) we see that $\widetilde{\mathfrak{p}}_{u}$ satisfies $\mathfrak{u}=\widetilde{\mathfrak{p}}_{u} \oplus \mathfrak{k}_{u}$, therefore we can define complex structures on $\widetilde{\mathfrak{p}}_{u}$, using the complex structures $I_{M}(o): T_{o}(M) \rightarrow T_{o}(M), J_{M}(o): T_{o}(M) \rightarrow T_{o}(M)$ on $T_{o}(M)$, as follows

$$
\begin{gathered}
\widetilde{I}: \widetilde{\mathfrak{p}}_{u} \rightarrow \widetilde{\mathfrak{p}}_{u} \quad \widetilde{J}: \widetilde{\mathfrak{p}}_{u} \rightarrow \widetilde{\mathfrak{p}}_{u} \\
\widetilde{I}=\left((\mathrm{d} \pi)_{\mid \widetilde{\mathfrak{p}}_{u}}\right)^{-1} \circ I_{M}(o) \circ(\mathrm{d} \pi)_{\widetilde{\mathfrak{p}}_{u}}, \quad \widetilde{J}=\left((\mathrm{d} \pi)_{\mid \widetilde{\mathfrak{p}}_{u}}\right)^{-1} \circ J_{M}(o) \circ(\mathrm{d} \pi)_{\mid \widetilde{\mathfrak{p}}_{u}} .
\end{gathered}
$$

We shall denote the complexifications of $\widetilde{I}$ and $\widetilde{J}$ by the same letters $\widetilde{I}: \widetilde{\mathfrak{p}} \rightarrow \widetilde{\mathfrak{p}}, \widetilde{J}: \widetilde{\mathfrak{p}} \rightarrow \widetilde{\mathfrak{p}}$. Let $\widetilde{\mathfrak{p}}^{+}$and $\widetilde{\mathfrak{p}}^{-}$be the i and (-i)-eigenspaces of $\widetilde{I}$, respectively. 
The following lemma relates $\widetilde{J}$ to $J$.

Lemma 4.19. Let $X \in \mathfrak{n}_{\mathfrak{p}_{u}}$ and let $J(X)=H+Y, \widetilde{J}(X)=\widetilde{H}+\widetilde{Y}$, where $H \in \mathfrak{h}_{\mathfrak{p}_{u}}, \widetilde{H} \in \mathfrak{q}$ and $Y, \widetilde{Y} \in \mathfrak{n}_{\mathfrak{p}_{u}}$ then $Y=\widetilde{Y}$. Furthermore, $H=0 \Longleftrightarrow \widetilde{H}=0$.

The same statement is valid if we replace $J$ with $I$ and $\widetilde{J}$ with $\widetilde{I}$.

Proof. By the definitions of $J$ and $\widetilde{J}$, we have

$$
\begin{aligned}
& \left((\mathrm{d} \pi)_{\mid \tilde{\mathfrak{p}}_{u}}\right)^{-1} \circ J_{M}(o) \circ(\mathrm{d} \pi)_{\mid \tilde{\mathfrak{p}}_{u}}(X)=\widetilde{H}+\widetilde{Y} \\
& \left((\mathrm{~d} \pi)_{\mid \mathfrak{p}_{u}}\right)^{-1} \circ J_{M}(o) \circ(\mathrm{d} \pi)_{\mid \mathfrak{p}_{u}}(X)=H+Y
\end{aligned}
$$

hence

$$
\mathrm{d} \pi(\widetilde{H}+\widetilde{Y})=\mathrm{d} \pi(H+Y)=J_{M}(o)(\mathrm{d} \pi(X)) .
$$

On the other hand $\mathfrak{k e r}(\mathrm{d} \pi)=\mathfrak{k}_{u}$, therefore $(H-\widetilde{H})+(Y-\widetilde{Y}) \in \mathfrak{k}_{u}$, hence $Y-\widetilde{Y} \in$ $\left(\mathfrak{k}_{u}+\mathfrak{h}_{u}\right) \cap \mathfrak{n}_{\mathfrak{p}_{u}}$. Since $\left(\mathfrak{k}_{u}+\mathfrak{h}_{u}\right) \cap \mathfrak{n}_{\mathfrak{p}_{u}}=\{0\}$, we see that $Y-\widetilde{Y}=0$ and

$$
(H-\widetilde{H}) \in \mathfrak{k}_{u} \text {. }
$$

Now if $H=0$ then $\widetilde{H} \in \mathfrak{k}_{u} \cap \mathfrak{q}=\{0\}$, hence $\widetilde{H}=0$. On the other hand if $\widetilde{H}=0$ then $H \in \mathfrak{k}_{u} \cap \mathfrak{h}_{\mathfrak{p}_{u}}=\{0\}$, hence $H=0$.

Lemma 4.20. The complex structure $\widetilde{I}$ satisfies the following conditions

$$
\begin{gathered}
\widetilde{I}\left(\mathfrak{n}_{\mathfrak{p}_{u}}\right)=\mathfrak{n}_{\mathfrak{p}_{u}}, \quad \widetilde{I}(\mathfrak{q})=\mathfrak{q} \\
\widetilde{\mathfrak{p}}^{+}=\left(\widetilde{\mathfrak{p}}^{+} \cap \mathfrak{h}\right)+\mathfrak{n}_{\mathfrak{p}}^{+} \quad \widetilde{\mathfrak{p}}^{-}=\left(\widetilde{\mathfrak{p}}^{-} \cap \mathfrak{h}\right)+\mathfrak{n}_{\mathfrak{p}}^{-}=\tau\left(\widetilde{\mathfrak{p}}^{+} \cap \mathfrak{h}\right)+\mathfrak{n}_{\mathfrak{p}}^{-} .
\end{gathered}
$$

Proof. The equalities $I\left(E_{\alpha}-E_{-\alpha}\right)=\mathrm{i}\left(E_{\alpha}+E_{-\alpha}\right), I\left(\mathrm{i}\left(E_{\alpha}+E_{-\alpha}\right)\right)=-\left(E_{\alpha}-E_{-\alpha}\right)$ are valid for $\alpha \in \Delta_{\mathfrak{p}}$ and from (99) we see that $I\left(\mathfrak{n}_{\mathfrak{p}_{u}}\right)=\mathfrak{n}_{\mathfrak{p}_{u}}$.

Hence, by the previous lemma $\widetilde{I}\left(\mathfrak{n}_{\mathfrak{p}_{u}}\right)=I(X)=\mathfrak{n}_{\mathfrak{p}_{u}}$.

Let us recall the fact that $I$ restricted to $\mathfrak{h} \cap \mathfrak{p}^{+}$is i and restricted to $\mathfrak{h} \cap \mathfrak{p}^{-}$is -i, therefore

$$
I(\mathfrak{h} \cap \mathfrak{p})=\mathfrak{h} \cap \mathfrak{p} \Rightarrow I\left(\mathfrak{h}_{\mathfrak{p}_{u}}\right)=\mathfrak{h}_{\mathfrak{p}_{u}} .
$$

Now we prove the second statement in (110). Let us take $H \in \mathfrak{q}, H \neq 0$. Let us decompose $\widetilde{I}(H)=H^{\prime}+B$, where $H^{\prime} \in \mathfrak{q}, B \in \mathfrak{n}_{\mathfrak{p}_{u}}$. We shall show that $B=0$. The definition of $\widetilde{I}$ implies $\left((\mathrm{d} \pi)_{\tilde{\mathfrak{p}}_{u}}\right)^{-1} \circ I_{M}(o) \circ(\mathrm{d} \pi)_{\widetilde{\mathfrak{p}}_{u}}(H)=H^{\prime}+B$, therefore

$$
(\mathrm{d} \pi)\left(H^{\prime}+B\right)=I_{M}(o)(\mathrm{d} \pi(H)) .
$$

Now since $H, H^{\prime} \in \mathfrak{h}_{u}$ and since $\mathfrak{h}_{u}=\mathfrak{h}_{\mathfrak{p}_{u}} \oplus \mathfrak{h}_{\mathfrak{k}_{u}}$, then we may decompose $H$ and $H^{\prime}$ as follows

$$
H=H_{1}+H_{2}, \quad H^{\prime}=H_{1}^{\prime}+H_{2}^{\prime} \quad H_{1}, H_{1}^{\prime} \in \mathfrak{h}_{\mathfrak{k}_{u}}, \quad H_{2}, H_{2}^{\prime} \in \mathfrak{h}_{\mathfrak{p}_{u}} .
$$

The equality (112) can be rewritten as follows

$$
(\mathrm{d} \pi)\left(H_{2}^{\prime}+B\right)=I_{M}(o)\left(\mathrm{d} \pi\left(H_{2}\right)\right)
$$

where we used $\mathfrak{k} \mathfrak{k r}(\mathrm{d} \pi)=\mathfrak{k}_{u}$ and $\mathfrak{h}_{\mathfrak{k}_{u}} \subset \mathfrak{k}_{u}$. Since $H_{2}^{\prime}+B \in \mathfrak{h}_{\mathfrak{p}_{u}}+\mathfrak{n}_{\mathfrak{p}_{u}}=\mathfrak{p}_{u}$ and $H_{2} \in \mathfrak{p}_{u}$, then

$$
\left(H_{2}^{\prime}+B\right)=\left((\mathrm{d} \pi)_{\mid \mathfrak{p}_{u}}\right)^{-1} \circ I_{M}(o) \circ(\mathrm{d} \pi)_{\mid \mathfrak{p}_{u}}\left(H_{2}\right)=I\left(H_{2}\right) .
$$


We have already shown that $I\left(\mathfrak{h}_{\mathfrak{p}_{u}}\right)=\mathfrak{h}_{\mathfrak{p}_{u}}$, which implies that $B=0$ and (110) is proved.

From (110) and Lemma 4.19 it follows that for each $X \in \mathfrak{n}_{\mathfrak{p}}$ we have $\widetilde{I}(X)=I(X)=\mathrm{i} X$, therefore

$$
\mathfrak{n}_{\mathfrak{p}}^{+} \subset \widetilde{\mathfrak{p}}^{+} \quad \mathfrak{n}_{\mathfrak{p}}^{-} \subset \widetilde{\mathfrak{p}}^{-} .
$$

Let us take any element $X \in \widetilde{\mathfrak{p}}^{+}$. By (109) we may decompose it as follows $X=A+H+B$, $A \in \mathfrak{n}_{\mathfrak{p}}^{-}, H \in \mathfrak{h} \cap \widetilde{\mathfrak{p}}, B \in \mathfrak{n}_{\mathfrak{p}}^{+}$. Since $A \in \mathfrak{n}_{\mathfrak{p}}^{-} \subset \widetilde{\mathfrak{p}}^{-}$and $B \in \mathfrak{n}_{\mathfrak{p}}^{+} \subset \widetilde{\mathfrak{p}}^{+}$, then

$$
\widetilde{I}(A+H+B)=-\mathrm{i} A+\widetilde{I}(H)+\mathrm{i} B .
$$

On the other hand since $A+H+B \in \widetilde{\mathfrak{p}}^{+}$, then $\widetilde{I}(A+H+B)=\mathrm{i}(A+H+B)$ hence

$$
-\mathrm{i} A+\widetilde{I}(H)+\mathrm{i} B=\mathrm{i} A+\mathrm{i} H+\mathrm{i} B
$$

this equality together with $\widetilde{I}(\mathfrak{h} \cap \widetilde{\mathfrak{p}})=\mathfrak{h} \cap \widetilde{\mathfrak{p}}$ implies that $A=0$, therefore $X=H+B$. On the other hand $B \in \mathfrak{n}_{\mathfrak{p}^{+}} \subset \widetilde{\mathfrak{p}}^{+}$therefore $X-B=H \in \widetilde{\mathfrak{p}}^{+} \cap \mathfrak{h}$. And we proved $\widetilde{\mathfrak{p}}^{+}=\left(\widetilde{\mathfrak{p}}^{+} \cap \mathfrak{h}\right)+\mathfrak{n}_{\mathfrak{p}}^{+}$. The other equality in (111) follows from $\widetilde{I} \circ \tau=\tau \circ \widetilde{I}$.

Let us choose a basis $\left\{U_{1}, U_{2}, \ldots, U_{m}\right\}$ of $\widetilde{\mathfrak{p}}^{+} \cap \mathfrak{h}$, then $\left\{V_{1}=\tau\left(U_{1}\right), V_{2}=\tau\left(U_{2}\right), \ldots, V_{m}=\right.$ $\left.\tau\left(U_{m}\right)\right\}$ is a basis of $\widetilde{\mathfrak{p}}^{-} \cap \mathfrak{h}$. Then by (111) the subspaces $\widetilde{\mathfrak{p}}^{+}, \widetilde{\mathfrak{p}}^{-}$decompose as follows

$$
\widetilde{\mathfrak{p}}^{+}=\operatorname{span}_{\mathbb{C}}\left\{U_{i}\right\}_{i=1}^{m}+\sum_{\alpha \in \Delta_{\mathfrak{p}}^{+}} \mathfrak{g}(\alpha) \quad \widetilde{\mathfrak{p}}^{-}=\operatorname{span}_{\mathbb{C}}\left\{V_{i}\right\}_{i=1}^{m}+\sum_{\alpha \in \Delta_{\mathfrak{p}}^{+}} \mathfrak{g}(-\alpha) .
$$

In the same way as in [6] (Definition 1.12, Proposition 1.13) one can prove, using the conditions $\widetilde{J}\left(\widetilde{\mathfrak{p}}^{+}\right)=\widetilde{\mathfrak{p}}^{-}, \widetilde{J}^{2}=-I d, \widetilde{J} \circ \tau=\tau \circ \widetilde{J}$, the following lemma

Lemma 4.21. There exist functions

$$
\begin{aligned}
\widetilde{\xi}:\{1,2, \ldots, m\} \times \Delta_{\mathfrak{p}}^{+} & \rightarrow \mathbb{C} \quad \widetilde{\mathbf{a}}: \Delta_{\mathfrak{p}}^{+} \times \Delta_{\mathfrak{p}}^{+} \rightarrow \mathbb{C} \\
\widetilde{\mathbf{b}}:\{1,2, \ldots, m\} \times\{1,2, \ldots, m\} \rightarrow \mathbb{C} \quad \widetilde{\eta}: \Delta_{\mathfrak{p}}^{+} \times\{1,2, \ldots, m\} & \rightarrow \mathbb{C}
\end{aligned}
$$

such that

$$
\begin{array}{ll}
\widetilde{J}\left(E_{\alpha}\right)=\sum_{t=1}^{m} \widetilde{\xi}_{t \alpha} V_{t}+\sum_{\beta \in \Delta_{\mathfrak{p}}^{+}} \widetilde{a}_{\beta \alpha} E_{-\beta} \quad \alpha \in \Delta_{\mathfrak{p}}^{+} \\
\widetilde{J}\left(U_{q}\right)=\sum_{t=1}^{m} \widetilde{b}_{t q} V_{t}+\sum_{\beta \in \Delta_{\mathfrak{p}}^{+}} \widetilde{\eta}_{\beta q} E_{-\beta} \quad q \in\{1, \ldots, m\} .
\end{array}
$$

The functions $\widetilde{\mathbf{a}}, \widetilde{\mathbf{b}}, \widetilde{\xi}, \widetilde{\eta}$ satisfy

$$
\overline{\widetilde{\mathbf{b}}} \widetilde{\xi}-\overline{\widetilde{\xi}} \widetilde{\mathbf{a}}=0, \quad \overline{\widetilde{\eta}} \xi-\overline{\widetilde{\mathbf{a}}} \widetilde{\mathbf{a}}=\mathrm{I}_{\Delta_{\mathfrak{p}}^{+}}, \quad \overline{\widetilde{\mathbf{a}}} \eta-\overline{\widetilde{\eta}} \widetilde{\mathbf{b}}=0, \quad \overline{\widetilde{\xi}} \widetilde{\eta}-\overline{\widetilde{\mathbf{b}}} \widetilde{\mathbf{b}}=\mathrm{I}_{m}
$$

Remark 4.22. The functions $\widetilde{\mathbf{a}}, \widetilde{\mathbf{b}}, \widetilde{\xi}, \widetilde{\eta}$ depend on the choice of the subspace $\mathfrak{q}$, which in turn determines $\widetilde{I}, \widetilde{J}$. For the case $\mathfrak{q}=\mathfrak{h}_{\mathfrak{p}_{u}}$, which gives the complex structures I, J, fixed in subsection 4.3, we shall denote these function by $\mathbf{a}, \mathbf{b}, \xi, \eta$ throughout section 4 . 
From Lemma 4.19 it follows

Lemma 4.23. The matrix $\widetilde{\mathbf{a}}$, assigned to $\widetilde{J}$ in accordance with Lemma 4.21, does not change as $\mathfrak{q}$ varies constrained to (108), i.e. $\widetilde{\mathbf{a}}=\mathbf{a}$ for any choice of $\mathfrak{q}$.

This lemma allows us to write $\mathbf{a}$ instead of $\widetilde{\mathbf{a}}$, without paying attention to the choice of $\mathfrak{q}$ and this matrix $\mathbf{a}$ is fixed throughout section 4 .

Corollary 4.24. Let $\alpha \in \Delta_{\mathfrak{p}}^{+}$then

$$
\widetilde{J}\left(E_{\alpha}\right) \in \mathfrak{h} \quad \Longleftrightarrow \quad \forall \zeta \in \Delta_{\mathfrak{p}}^{+} \quad a_{\zeta \alpha}=0 .
$$

Remark 4.25. It is convenient to extend the definitions of the functions $\mathbf{a}, \tilde{\eta}$. Let $\alpha, \beta \in$ $\mathfrak{h}^{*}, \quad k \in \mathbb{N}$. If $\alpha \notin \Delta_{\mathfrak{p}}^{+}$or $\beta \notin \Delta_{\mathfrak{p}}^{+}$, then we set $a_{\alpha, \beta}=\widetilde{\eta}_{\alpha, k}=0$.

Definition 4.26. In this subsection we started from any subspace $\mathfrak{q} \subset \mathfrak{h}_{u}$, satisfying (108) and constructed a 6-uple $\left(\widetilde{\mathfrak{p}}, \widetilde{\mathfrak{p}}^{+}, \widetilde{J}, \widetilde{\xi}, \widetilde{\eta}, \widetilde{\mathbf{b}}\right)$, depending on $\mathfrak{q}$ (we recall that the matrix a does not depend on $\mathfrak{q})$. For the sake of brevity we shall call such a 6 -uple $(\widetilde{\mathfrak{p}}, \widetilde{\mathfrak{p}}+, \widetilde{J}, \widetilde{\xi}, \widetilde{\eta}, \widetilde{\mathbf{b}})$ Cartan variation of $\mathfrak{p}_{u}$. Of course Lemma 4.20 and Corollary 4.24 are valid for any Cartan variation of $\mathfrak{q}$. Furthermore, $\left(\mathfrak{p}, \mathfrak{p}^{+}, J, \xi, \eta, \mathbf{b}\right)$ is a Cartan variation of $\mathfrak{p}_{u}$ (see (98)).

4.9.2. Equations for $\mathbf{a}, \widetilde{\mathbf{b}}, \widetilde{\xi}, \widetilde{\eta}$. The following theorem is well known (see e.g. [10], Ch. X)

Theorem 4.27. Let $\mathbf{U}$ be a connected Lie group with Lie algebra $\mathfrak{u}$. Let $\mathbf{K}_{u}$ be a closed subgroup of $\mathbf{U}$ with Lie algebra $\mathfrak{k}_{u}$. Let $J_{M}$ be a left-invariant integrable complex structure on the coset space $M=\mathbf{U} / \mathbf{K}_{u}$. Let $\widetilde{\mathfrak{p}}_{u}$ be any subspace of $\mathfrak{u}$ such that

$$
\mathfrak{u}=\mathfrak{k}_{u} \oplus \widetilde{\mathfrak{p}}_{u} .
$$

Then the complex structure on $\widetilde{\mathfrak{p}}_{u}$, defined as follows

$$
\widetilde{J}=\left((\mathrm{d} \pi)_{\mid \widetilde{\mathfrak{p}}_{u}}\right)^{-1} \circ J_{M}(o) \circ(\mathrm{d} \pi)_{\mid \widetilde{\mathfrak{p}}_{u}}
$$

satisfies the following equation

$$
\forall X, Y \in \widetilde{\mathfrak{p}}_{u} \quad[\widetilde{J}(X), \widetilde{J}(Y)]_{\widetilde{\mathfrak{p}}_{u}}-[X, Y]_{\widetilde{\mathfrak{p}}_{u}}-\widetilde{J}\left([X, \widetilde{J}(Y)]_{\widetilde{\mathfrak{p}}_{u}}\right)-\widetilde{J}\left([\widetilde{J}(X), Y]_{\widetilde{\mathfrak{p}}_{u}}\right)=0 .
$$

Let us remark that in [10] the reductivity condition $\left[\mathfrak{k}_{u}, \widetilde{\mathfrak{p}}_{u}\right] \subset \widetilde{\mathfrak{p}}_{u}$ is presumed, but it is used there for other statements. One can verify that for the proof of the theorem above, this condition is not necessary.

We apply this theorem to the complex structure $\widetilde{J}$ in any Cartan variation $\left(\widetilde{\mathfrak{p}}, \widetilde{\mathfrak{p}}^{+}, \widetilde{J}, \widetilde{\xi}, \widetilde{\eta}, \widetilde{\mathbf{b}}\right)$ of $\mathfrak{p}_{u}$ and from (116) we infer

$$
\forall X, Y \in \widetilde{\mathfrak{p}} \quad[\widetilde{J}(X), \widetilde{J}(Y)]_{\tilde{\mathfrak{p}}}-[X, Y]_{\mid \widetilde{\mathfrak{p}}}-\widetilde{J}\left([X, \widetilde{J}(Y)]_{\mid \widetilde{\mathfrak{p}}}\right)-\widetilde{J}\left([\widetilde{J}(X), Y]_{\mid \widetilde{\mathfrak{p}}}\right)=0 .
$$

Using this equation, we prove:

Proposition 4.28. Let $\alpha, \beta \in \Delta_{\mathfrak{p}}^{+}, \quad q=1, \ldots, m$. Then

$$
a_{\beta, \alpha}(\alpha+\beta)\left(U_{q}\right)=p r_{\mathfrak{g}(-\beta)}\left(\sum_{\nu \in \Delta_{\mathfrak{p}}^{+}} \widetilde{\eta}_{\nu, q}\left[E_{-\nu}, E_{\alpha}\right]\right) .
$$


Proof. We put in (117) $X=U_{q}, Y=E_{\alpha}$, apply $\widetilde{J}$ on the equality and expand, using (115) and keeping explicit only the coefficient before $E_{-\beta}$. The computation is the same as in [6], Proposition 4.1.

Proposition 4.29. Let $\alpha, \beta, \gamma \in \Delta_{\mathfrak{p}}^{+}, \widetilde{J} E_{\gamma} \in \mathfrak{h}$. Then

$$
a_{\beta, \alpha}(\beta+\alpha)\left(\widetilde{J}\left(E_{-\gamma}\right)\right)=p r_{\mathfrak{g}(-\beta)}\left(\left[E_{\alpha}, E_{-\gamma}\right]\right) .
$$

Proof. We put in (117) $X=E_{-\gamma}, Y=E_{\alpha}$ and expand, using (115) and keeping explicit only terms with component in $\mathfrak{n}^{-}$. The computation is the same as in [6], Proposition 4.9.

Proposition 4.30. Let $\alpha, \beta, \gamma \in \Delta_{\mathfrak{p}}^{+}, \widetilde{J} E_{\gamma} \in \mathfrak{h}$. Let $\forall \zeta \in \Delta_{\mathfrak{p}}^{+} a_{\gamma \zeta}=a_{\zeta \gamma}=0$ and $\beta+\alpha \neq \gamma$, then

$$
\sum_{\zeta \in \Phi_{\gamma}^{\mathfrak{p}}} a_{\zeta \beta} N_{\gamma,-\zeta} a_{\alpha, \gamma-\zeta}=0 .
$$

Proof. We just put in (117) $X=E_{\gamma}, Y=E_{\beta}$ and use (115). Keeping explicit only terms with component in $\mathfrak{n}^{-}$, by the same computation as in [6], Lemma 4.11 we obtain]:

$$
a_{\alpha, \beta}(\alpha+\beta)\left(\widetilde{J} E_{\gamma}\right)+\sum_{\nu \in \Phi_{\gamma}^{\mathfrak{p}}} N_{\gamma,-\nu} a_{\nu, \beta} a_{\alpha, \gamma-\nu}=0 .
$$

We recall that the notation $\Phi_{\gamma}^{\mathfrak{p}}$ is in (95).

When $\beta+\alpha \neq \gamma$, Proposition 4.29 gives $8 a_{\alpha, \beta}(\alpha+\beta)\left(\widetilde{J} E_{\gamma}\right)=0$, whence the lemma.

From these propositions we derive some useful corollaries.

Corollary 4.31. Let $\left(\widetilde{\mathfrak{p}}, \widetilde{\mathfrak{p}}^{+}, \widetilde{J}, \widetilde{\xi}, \widetilde{\eta}, \widetilde{\mathbf{b}}\right)$ be any Cartan variation of $\mathfrak{p}_{u}$. Let $\gamma \in \Delta_{\mathfrak{p}}^{+}$be such that $\widetilde{J}\left(E_{\gamma}\right) \in \mathfrak{h}$. Let $\alpha, \beta \in \Delta_{\mathfrak{p}}^{+}$be such that $\alpha+\beta=\gamma$. Then $\gamma\left(\widetilde{J}\left(E_{\gamma}\right)\right) \neq 0$, $a_{\alpha \beta} \neq 0$, $a_{\beta \alpha} \neq 0$ and

$$
a_{\alpha \beta}=\frac{N_{\gamma,-\beta}}{\gamma\left(\widetilde{J}\left(E_{\gamma}\right)\right)} \quad a_{\beta \alpha}=\frac{N_{\gamma,-\alpha}}{\gamma\left(\widetilde{J}\left(E_{\gamma}\right)\right)} .
$$

Proof. Since $\alpha, \beta, \gamma \in \Delta_{\mathfrak{p}}^{+}, \widetilde{J} E_{\gamma} \in \mathfrak{h}$ and $\alpha+\beta=\gamma$ then by (119) in Proposition 4.29

$$
a_{\beta \alpha} \gamma\left(\widetilde{J} E_{-\gamma}\right)=N_{\alpha,-\gamma}=N_{\gamma,-\alpha} \quad a_{\alpha \beta} \gamma\left(\widetilde{J} E_{-\gamma}\right)=N_{\beta,-\gamma}=N_{\gamma,-\beta}
$$

and the corollary follows.

Corollary 4.32. Let $\left(\widetilde{\mathfrak{p}}, \widetilde{\mathfrak{p}}^{+}, \widetilde{J}, \widetilde{\xi}, \widetilde{\eta}, \widetilde{\mathbf{b}}\right)$ be any Cartan variation of $\mathfrak{p}_{u}$. Let $\alpha, \beta \in \Delta_{\mathfrak{p}}^{+}$and $\alpha+\beta$ does not vanish on $\widetilde{\mathfrak{p}} \cap \mathfrak{h}$. Then

\footnotetext{
7 now in the conditions of the proposition we have $\forall \zeta \in \Delta_{\mathfrak{p}}^{+} a_{\gamma, \zeta}=a_{\zeta, \gamma}=0$, because in the computation we need $a_{\gamma, \beta}=0$ and we have not proved yet that the vanishing of $a_{\beta, \gamma}$ implies $a_{\gamma, \beta}=0$

${ }^{8}$ Because $(\alpha+\beta)\left(\widetilde{J} E_{\gamma}\right)=\overline{(\alpha+\beta)\left(\widetilde{J} E_{-\gamma}\right)}$.
} 
(i) $a_{\alpha \beta}=0 \Longleftrightarrow a_{\beta \alpha}=0$,

(ii) If $\alpha+\beta \notin \Delta_{\mathfrak{p}}^{+}$, then $a_{\alpha \beta}=a_{\beta \alpha}=0$.

Proof. Now $\left\{U_{1}, \ldots, U_{m}, V_{1}, \ldots, V_{m}\right\}$ is a basis of $\widetilde{\mathfrak{p}} \cap \mathfrak{h}$ and we have $\delta\left(V_{i}\right)=-\overline{\delta\left(U_{i}\right)}$ for any $\delta \in \Delta$. Since $\alpha+\beta$ does not vanish on $\widetilde{\mathfrak{p}} \cap \mathfrak{h}$ then $(\alpha+\beta)\left(U_{q}\right) \neq 0$ for some $q \in\{1, \ldots, m\}$. Then the lemma follows from (118) and the formula

$$
\operatorname{pr}_{\mathfrak{g}(-\beta)}\left(\sum_{\nu \in \Delta_{\mathfrak{p}}^{+}} \widetilde{\eta}_{\nu, q}\left[E_{-\nu}, E_{\alpha}\right]\right)=\left\{\begin{array}{clc}
0 & \text { if } & \alpha+\beta \notin \Delta_{\mathfrak{p}}^{+} \\
\widetilde{\eta}_{\alpha+\beta, q} N_{-\alpha-\beta, \alpha} & \text { if } & \alpha+\beta \in \Delta_{\mathfrak{p}}^{+}
\end{array}\right.
$$

In particular we see that $p r_{\mathfrak{g}(-\beta)}\left(\sum_{\nu \in \Delta_{\mathfrak{p}}^{+}} \widetilde{\eta}_{\nu, q}\left[E_{-\nu}, E_{\alpha}\right]\right)=0$ iff $p r_{\mathfrak{g}(-\alpha)}\left(\sum_{\nu \in \Delta_{\mathfrak{p}}^{+}} \widetilde{\eta}_{\nu, q}\left[E_{-\nu}, E_{\beta}\right]\right)=$ 0 .

4.10. Results for J. Determining of the subsets $\Delta_{\mathfrak{k}}^{+}, \Delta_{\mathfrak{p}}^{+}$. In subsection 4.9 we obtained some results for $\widetilde{J}$, related to any Cartan variation $\left(\widetilde{\mathfrak{p}}, \widetilde{\mathfrak{p}}^{+}, \widetilde{J}, \widetilde{\xi}, \widetilde{\eta}, \widetilde{\mathbf{b}}\right)$ of $\mathfrak{p}_{u}$. In this subsection we resume to analyse the complex structures $I$ and $J$ defined in subsection 4.3 . In particular we calculate the coefficients $\left\{a_{\alpha \beta}\right\}_{\left\{\alpha, \beta \in \Delta_{\mathfrak{p}}^{+}\right\}}$, defined in subsection 4.9, Remark 4.22. Furthermore, we describe the structure of $\Delta_{\mathfrak{k}}^{+}$and $\Delta_{\mathfrak{p}}^{+}$, using the stem $\Gamma$.

We start with a Lemma

Lemma 4.33. Let $\gamma \in \Gamma_{\mathfrak{p}}$ be a maximal root. Then

$$
\begin{gathered}
\forall \zeta \in \Delta_{\mathfrak{p}}^{+} \quad a_{\zeta \gamma}=a_{\gamma \zeta}=0 \\
W_{\gamma} \in \mathfrak{p}_{u}, \quad \Phi_{\gamma}^{+} \subset \Delta_{\mathfrak{p}}^{+} \\
\forall \alpha \in \Phi_{\gamma}^{+} \forall \beta \in \Delta_{\mathfrak{p}}^{+} \quad\left(a_{\alpha \beta} \neq 0 \text { or } a_{\beta \alpha} \neq 0\right) \Longleftrightarrow \alpha+\beta=\gamma .
\end{gathered}
$$

Proof. We have $\alpha\left(H_{\gamma}\right)>0$ for $\alpha \in \Phi_{\gamma}^{+}$(see Proposition 2.1. (9)) and $\gamma\left(H_{\gamma}\right)=2$. Since $\gamma$ is a maximal root then $\alpha\left(H_{\gamma}\right)=0$ for $\alpha \in \Delta^{+} \backslash\left(\Phi_{\gamma}^{+} \cup\{\gamma\}\right)$ (Proposition 2.1, (17) and (8)). Hence we see that

$$
\forall \alpha \in \Delta_{\mathfrak{p}}^{+} \quad(\gamma+\alpha)\left(H_{\gamma}\right) \neq 0 .
$$

Now, since $\gamma \in \Gamma_{\mathfrak{p}}$ is a maximal root, Lemma 4.16 implies that $H_{\gamma} \notin \mathfrak{k}$. We recall that $\mathfrak{h}_{u}=\mathfrak{h} \cap \mathfrak{u}$ and $\mathfrak{h}_{\mathfrak{k}_{u}}=\mathfrak{h}_{u} \cap \mathfrak{k}_{u}$ (see (97)). Since $H_{\gamma} \notin \mathfrak{k}$ then $W_{\gamma} \notin \mathfrak{h}_{\mathfrak{k}_{u}}$, therefore we can choose $\mathfrak{q}$ such that

$$
\mathfrak{h}_{u}=\mathfrak{q} \oplus \mathfrak{h}_{\mathfrak{k}_{u}} \quad W_{\gamma} \in \mathfrak{q}
$$

From this $\mathfrak{q}$ we obtain a Cartan variation of $\mathfrak{p}_{u}\left(\widetilde{\mathfrak{p}}, \widetilde{\mathfrak{p}}^{+}, \widetilde{J}, \widetilde{\xi}, \widetilde{\eta}, \widetilde{\mathbf{b}}\right)$ as in Definition 4.26. Now (125) and (126) show that for any $\zeta \in \Delta_{\mathfrak{p}}^{+}$the functional $(\zeta+\gamma)$ does not vanish on $\mathfrak{h} \cap \widetilde{\mathfrak{p}}$, hence Lemma 4.32, (ii) is applicable. Therefore, $\gamma$ being a maximal root, we proved (122). Therefore by Corollary 4.24 $J\left(E_{\gamma}\right) \in \mathfrak{h}$ and Lemma 4.17] implies that $W_{\gamma} \in \mathfrak{p}_{u}$. On the other hand Lemma 4.18 implies that $\Phi_{\gamma}^{+} \subset \Delta_{\mathfrak{p}}^{+}$. 
Since $\Phi_{\gamma}^{+} \subset \Delta_{\mathfrak{p}}^{+}$, then $\Phi_{\gamma}^{\mathfrak{p}}=\Phi_{\gamma}^{+}$. To prove (124), we first show that

$$
\forall \zeta, \delta \in \Phi_{\gamma}^{+} \cup\{\gamma\} \quad a_{\zeta \delta} \neq 0 \Longleftrightarrow \zeta+\delta=\gamma .
$$

Indeed, for any $\zeta, \delta \in \Phi_{\gamma}^{+} \cup\{\gamma\}$ we have $\zeta\left(H_{\gamma}\right)>0, \delta\left(H_{\gamma}\right)>0$ and then Corollary 4.32, (ii), Corollary 4.31 in conjunction with the well known property (see Proposition 2.1, ([6])):

$$
\forall \zeta, \delta \in \Phi_{\gamma}^{+} \cup\{\gamma\} \quad \zeta+\delta \in \Delta^{+} \Longleftrightarrow \zeta+\delta=\gamma
$$

imply formula (127).

Now we take any $\alpha \in \Phi_{\gamma}^{+}$and $\beta \in \Delta_{\mathfrak{p}}^{+}$. Since we have proved (127), we may think that $\beta \notin \Phi_{\gamma}^{+} \cup\{\gamma\}$. Now we apply Theorem 4.30, (120), as we take into account (122) and $\alpha+\beta \neq \gamma$, since $\beta \notin \Phi_{\gamma}^{+}$, and obtain

$$
\sum_{\zeta \in \Phi_{\gamma}^{+}} a_{\zeta \beta} N_{\gamma,-\zeta} a_{\alpha, \gamma-\zeta}=0 .
$$

From (127) it follows that for $\zeta \in \Phi_{\gamma}^{+} a_{\alpha, \gamma-\zeta} \neq 0$ if and only if $\zeta=\alpha$. Therefore $a_{\alpha \beta}=0$.

To prove $a_{\beta \alpha}=0$ we apply Corollary 4.32 (i) (we may apply it since $\alpha\left(H_{\gamma}\right)>0, \beta\left(H_{\gamma}\right) \geq$ $0)$. The lemma is completely proved.

In order that we prove the main theorem of this subsection: Theorem (4.35), we need of some preliminary discussion on $\Delta_{\mathfrak{p}}^{+}$and $\Gamma_{\mathfrak{p}}$.

Lemma 4.34. The subset $\Delta_{\mathfrak{p}}^{+}$is nonempty iff $\Gamma_{\mathfrak{p}}$ (for the notation $\Gamma_{\mathfrak{p}}$ see (96)) contains a maximal root. Besides, for any $\gamma \in \Gamma$ and $\alpha \in \Phi_{\gamma}^{+}$the inclusion $\alpha \in \Delta_{\mathfrak{p}}^{+}$implies $\gamma \in \Gamma_{\mathfrak{p}}$, $i$. $e$.

$$
\Delta_{\mathfrak{p}}^{+}=\bigcup_{\gamma \in \Gamma_{\mathfrak{p}}}\left(\Phi_{\gamma}^{+} \cup\{\gamma\}\right) \cap \Delta_{\mathfrak{p}}^{+}
$$

Proof. Let us recall that we have disjoint union $\Delta^{+}=\Delta_{\mathfrak{p}}^{+} \cup \Delta_{\mathfrak{k}}^{+}$(see (87)) .

Let $\gamma \in \Gamma, \alpha \in \Phi_{\gamma}^{+}$and $\alpha \in \Delta_{\mathfrak{p}}^{+}$. By Lemma 4.13 $\gamma \in \Delta^{+} \backslash \Delta_{\mathfrak{k}}^{+}=\Delta_{\mathfrak{p}}^{+}$, hence $\gamma \in \Gamma_{\mathfrak{p}}$ (recall that $\left.\Gamma_{\mathfrak{p}}=\Gamma \cap \Delta_{\mathfrak{p}}^{+}\right)$.

Thus we see that for any $\gamma \in \Gamma$ and $\alpha \in \Phi_{\gamma}^{+}$the inclusion $\alpha \in \Delta_{\mathfrak{p}}^{+}$implies $\gamma \in \Gamma_{\mathfrak{p}}$.

Now (128) follows from the partition (4).

If $\Gamma_{\mathfrak{p}}$ does not contain a maximal root, then all the maximal roots are in $\Gamma_{\mathfrak{k}}=\Gamma \backslash \Gamma_{\mathfrak{p}}$. On the other hand from (10) in Proposition 2.1 we know that for any root $\gamma \in \Gamma$ there exists a maximal root $\widetilde{\gamma} \in \Gamma$, such that $\gamma \succeq \widetilde{\gamma}$. All the maximal roots being in $\Gamma_{\mathfrak{k}}$, we see that for any root $\gamma \in \Gamma$ there exists a maximal root $\widetilde{\gamma} \in \Gamma_{\mathfrak{k}}$, such that $\gamma \succeq \widetilde{\gamma}$. Hence Lemma 4.13 implies that $\Delta^{+}=\Delta_{\mathfrak{k}}^{+}$, therefore $\Delta_{\mathfrak{p}}^{+}=\emptyset$.

This lemma shows that if $\Gamma_{\mathfrak{p}}$ is non empty, then the elements of $\Gamma_{\mathfrak{p}}$ are a subsequence of $\gamma_{1}, \ldots, \gamma_{d}$, whose first element is a maximal root (we recall that the maximal roots are in the

\footnotetext{
${ }^{9}$ for the notation $\Phi_{\gamma}^{\mathfrak{p}}$ see (95)
} 
beginning of $\gamma_{1}, \ldots, \gamma_{d}$ - see Corollary 2.2). Let this subsequence be $\gamma_{i_{1}}, \ldots, \gamma_{i_{\mathfrak{p}}}$ with length $d_{\mathfrak{p}}$ (here $\left.1 \leq i_{1}<i_{2}<\cdots<i_{d_{\mathfrak{p}}} \leq d\right)$. For the sake of brevity, we shall denote

$$
\mu_{1}=\gamma_{i_{1}}, \quad \mu_{2}=\gamma_{i_{2}}, \ldots, \quad \mu_{d_{\mathfrak{p}}}=\gamma_{i_{d_{\mathfrak{p}}}} .
$$

With these notations we represent $\Gamma_{\mathfrak{p}}$, if it is nonempty, as a sequence $\Gamma_{\mathfrak{p}}=\left\{\mu_{1}, \mu_{2}, \ldots, \mu_{d_{\mathfrak{p}}}\right\}$, such that $\mu_{1}$ is a maximal root and if $\mu_{i}, \mu_{j} \in \Gamma_{\mathfrak{p}}$, then

$$
\mu_{i} \prec \mu_{j} \Rightarrow i<j \text {. }
$$

Theorem 4.35. Let $\gamma \in \Gamma_{\mathfrak{p}}$, then $J\left(E_{\gamma}\right) \in \mathfrak{h}$ and $W_{\gamma} \in \mathfrak{p}_{u}$. Furthermore

$$
\begin{gathered}
\Delta_{\mathfrak{p}}^{+}=\bigcup_{\gamma \in \Gamma_{\mathfrak{p}}} \Phi_{\gamma}^{+} \cup\{\gamma\} \\
\forall \alpha \in \Delta_{\mathfrak{p}}^{+} \forall \beta \in \Delta_{\mathfrak{p}}^{+} \quad a_{\alpha \beta} \neq 0 \Longleftrightarrow \alpha+\beta \in \Gamma_{\mathfrak{p}} .
\end{gathered}
$$

Proof. We represented $\Gamma_{\mathfrak{p}}$ as a sequence $\mu_{1}, \mu_{2}, \ldots, \mu_{d_{\mathfrak{p}}}$, where $\mu_{1}$ is a maximal root.

We shall prove the theorem by induction with respect to the integer $1 \leq i \leq d_{\mathfrak{p}}$. Let us assume that for some $1 \leq i \leq d_{\mathfrak{p}}-1$ the following holds

$$
\begin{array}{cl}
\forall j \in\{1, \ldots, i\} & \left(H_{\mu_{j}} \in \mathfrak{p} \text { and } \Phi_{\mu_{j}}^{\mathfrak{p}}=\Phi_{\mu_{j}}^{+} \subset \Delta_{\mathfrak{p}}^{+}\right. \text {and } \\
\left(\forall \alpha \in \Phi_{\mu_{j}}^{+} \cup\left\{\mu_{j}\right\} \forall \beta \in \Delta_{\mathfrak{p}}^{+}\right. & \left.\left.\left(a_{\alpha \beta} \neq 0 \text { or } a_{\beta \alpha} \neq 0\right) \Longleftrightarrow \alpha+\beta=\mu_{j}\right)\right) .
\end{array}
$$

If $i=1$, then (132) holds by Lemma 4.33 (since $\mu_{1}$ is a maximal root).

Now we consider the case $i+1$.

First, we shall prove that $H_{\mu_{i+1}} \notin \mathfrak{k}$. Indeed, let us assume that $H_{\mu_{i+1}} \in \mathfrak{k}$. Then, since (see (129) and Proposition 2.1)

$$
\forall \alpha \in \bigcup_{j=i+1}^{d_{\mathfrak{p}}}\left(\Phi_{\mu_{j}}^{+} \cup\left\{\mu_{j}\right\}\right) \cap \Delta_{\mathfrak{p}}^{+} \quad \alpha\left(H_{\mu_{i+1}}\right) \geq 0,
$$

by Lemma 4.15 we should obtain (recall the equality (128) in Lemma 4.34 also)

$$
J\left(E_{\mu_{i+1}}\right)=\sum_{\left\{\alpha \in \Phi_{\mu_{j}}^{+} \cup\left\{\mu_{j}\right\} ; 1 \leq j \leq i\right\}} a_{\alpha, \mu_{i+1}} E_{-\alpha} .
$$

On the other hand the induction assumption (132) shows that for $\alpha \in \Phi_{\mu_{j}}^{+} \cup\left\{\mu_{j}\right\}, 0 \leq j \leq i$ we have $a_{\alpha, \mu_{i+1}}=0$ and we see that $H_{\mu_{i+1}} \in \mathfrak{k}$ implies $J\left(E_{\mu_{i+1}}\right)=0$, which contradicts $J^{2}=-I d$.

Therefore $H_{\mu_{i+1}} \notin \mathfrak{k}$ and we can choose $\mathfrak{q}$ such that

$$
\mathfrak{h}_{u}=\mathfrak{q} \oplus \mathfrak{h}_{\mathfrak{k}_{u}} \quad W_{\mu_{i+1}} \in \mathfrak{q} .
$$

From this $\mathfrak{h}_{\widetilde{\mathfrak{p}}_{\mathfrak{u}}}$ we obtain a Cartan variation of $\mathfrak{p}_{u}\left(\widetilde{\mathfrak{p}}, \widetilde{\mathfrak{p}}^{+}, \widetilde{J}, \widetilde{\xi}, \widetilde{\eta}, \widetilde{\mathbf{b}}\right)$ as in Definition 4.26. 
By (133) we see that for $\alpha \in\left(\Phi_{\mu_{j}}^{+} \cup\left\{\mu_{j}\right\}\right) \cap \Delta_{\mathfrak{p}}^{+}, i+1 \leq j \leq d_{\mathfrak{p}}$ the functional $\left(\alpha+\mu_{i+1}\right)$ does not vanish on $\mathfrak{h} \cap \widetilde{\mathfrak{p}}$, besides for such type of $\alpha$ we have $\alpha+\mu_{i+1} \notin \Delta$, hence Corollary 4.32, (ii) is applicable to $\alpha$ and $\mu_{i+1}$. Therefore

$$
\forall \alpha \in \bigcup_{j=i+1}^{d_{\mathfrak{p}}}\left(\Phi_{\mu_{j}}^{+} \cup\left\{\mu_{j}\right\}\right) \cap \Delta_{\mathfrak{p}}^{+} \quad a_{\alpha, \mu_{i+1}}=a_{\mu_{i+1}, \alpha}=0 .
$$

The last relation together with the induction assumption (132) and equality (128) in Lemma 4.34 imply

$$
\forall \alpha \in \Delta_{\mathfrak{p}}^{+} \quad a_{\alpha, \mu_{i+1}}=a_{\mu_{i+1}, \alpha}=0 .
$$

Therefore $J\left(E_{\mu_{i+1}}\right) \in \mathfrak{h}$ and Lemmas 4.17 and 4.18 imply

$$
W_{\mu_{i+1}} \in \mathfrak{p}_{u} \quad \Phi_{\mu_{i+1}}^{\mathfrak{p}}=\Phi_{\mu_{i+1}}^{+} \subset \Delta_{\mathfrak{p}}^{+} .
$$

Now since $H_{\mu_{i+1}} \in \mathfrak{p}$ then for any pair $\zeta, \delta \in \Phi_{\mu_{i+1}}^{+} \cup\left\{\mu_{i+1}\right\}$ the functional $\zeta+\delta$ does not vanish on $\mathfrak{p} \cap \mathfrak{h}$, besides we know that for such a pair the relation $\zeta+\delta \in \Delta$ holds iff $\zeta+\delta=\mu_{i+1}$. Therefore Corollary 4.32, (ii) and Corollary 4.31 imply

$$
\forall \zeta, \delta \in \Phi_{\mu_{i+1}}^{+} \cup\left\{\mu_{i+1}\right\} \quad a_{\zeta \delta} \neq 0 \Longleftrightarrow \zeta+\delta=\mu_{i+1} .
$$

In order that we complete the induction, it remains to prove that for any $\alpha \in \Phi_{\mu_{i+1}}^{+}$and $\beta \in \Delta_{\mathfrak{p}}^{+} \backslash\left(\Phi_{\mu_{i+1}}^{+} \cup\left\{\mu_{i+1}\right\}\right)$ the equalities $a_{\alpha \beta}=a_{\beta \alpha}=0$ are valid. To that end we employ Theorem 4.30, (120), as we take into account (135) and $\alpha+\beta \neq \mu_{i+1}$, since $\beta \notin \Phi_{\mu_{i+1}}^{+}$, and obtain

$$
\sum_{\zeta \in \Phi_{\mu_{i+1}^{+}}^{+}} a_{\zeta \beta} N_{\mu_{i+1},-\zeta} a_{\alpha, \mu_{i+1}-\zeta}=0
$$

By (137) it follows that, as $\zeta$ varies through $\Phi_{\mu_{i+1}}^{+}, a_{\alpha, \mu_{i+1}-\zeta} \neq 0$ iff $\zeta=\alpha$ and therefore the equation above is reduced to $a_{\alpha \beta}=0$. To prove that $a_{\beta \alpha}=0$ we can use Corollary 4.32, (i), provided that $\alpha+\beta$ does not vanish on $\mathfrak{p} \cap \mathfrak{h}$. The nonvanishing of $\alpha+\beta$ for $\beta \in \Phi_{\mu_{j}}^{+} \cup\left\{\mu_{j}\right\}$, $j \geq i+1$ follows from $\alpha\left(H_{\mu_{i+1}}\right)>0$ and $\beta\left(H_{\mu_{i+1}}\right) \geq 0$ (here we use (136)). The nonvanishing of $\alpha+\beta$ for $\beta \in \Phi_{\mu_{j}}^{+} \cup\left\{\mu_{j}\right\}, j \leq i$ follows from $\alpha\left(H_{\mu_{j}}\right) \geq 0$ and $\beta\left(H_{\mu_{j}}\right)>0$ (here we use that by the induction assumption (132) $H_{\mu_{j}} \in \mathfrak{p}$ ).

Thus far, we proved by induction that for any $\gamma \in \Gamma_{\mathfrak{p}}$, we have $J\left(E_{\gamma}\right) \in \mathfrak{h}, H_{\gamma} \in \mathfrak{p}$ and

$$
\begin{gathered}
\Delta_{\mathfrak{p}}^{+}=\bigcup_{\gamma \in \Gamma_{\mathfrak{p}}} \Phi_{\gamma}^{+} \cup\{\gamma\} \\
\forall \alpha \in \Delta_{\mathfrak{p}}^{+} \forall \beta \in \Delta_{\mathfrak{p}}^{+} \quad\left(a_{\alpha \beta} \neq 0 \text { or } a_{\beta \alpha} \neq 0\right) \Longleftrightarrow \alpha+\beta \in \Gamma_{\mathfrak{p}} .
\end{gathered}
$$

Now Corollary 4.31 shows that the theorem follows.

Corollary 4.36. $\mathfrak{k}$ is a $\Gamma_{\mathfrak{k}}$-stemmed subalgebra of $\mathfrak{g}$. 
Proof. By Theorem 4.35 and Corollary 4.14 it follows that the subsets $\Delta_{\mathfrak{k}}, \Delta_{\mathfrak{k}}^{+}$are of the type

$$
\begin{aligned}
\Delta_{\mathfrak{k}}^{+} & =\bigcup_{\gamma \in \Gamma_{\mathfrak{k}}} \Phi_{\gamma}^{+} \cup\{\gamma\}, \\
\Delta_{\mathfrak{k}} & =\bigcup_{\gamma \in \Gamma_{\mathfrak{k}}} \Phi_{\gamma} \cup\{\gamma\},
\end{aligned}
$$

where $\Gamma_{\mathfrak{k}}$ is a substem of $(\Gamma, \prec)$. Now the decomposition (82) shows that $\mathfrak{k}$ is a $\Gamma_{\mathfrak{k}}$-subalgebra.

Now, after having proved Theorem 4.35, by the same ideas as in [6] we shall describe the complex structures $I$ and $J$ more explicitely. To that end now we introduce notaions (additional to (97)):

$$
\begin{gathered}
\mathfrak{o}_{\mathfrak{p}}=\mathfrak{p} \cap \mathfrak{o}, \quad \mathfrak{o}_{\mathfrak{k}}=\mathfrak{k} \cap \mathfrak{o}, \quad \mathfrak{w}_{\mathfrak{p}}=\operatorname{span}_{\mathbb{R}}\left\{W_{\gamma} \mid \gamma \in \Gamma_{\mathfrak{p}}\right\} \\
\mathfrak{h}_{\mathfrak{p}}=\mathfrak{h} \cap \mathfrak{p}, \quad \mathfrak{h}_{\mathfrak{p}}^{+}=\mathfrak{h} \cap \mathfrak{p}^{+}, \quad \mathfrak{h}_{\mathfrak{p}}^{-}=\mathfrak{h} \cap \mathfrak{p}^{-} \\
\mathfrak{w}_{\mathfrak{p}}=\operatorname{span}_{\mathbb{R}}\left\{W_{\gamma} \mid \gamma \in \Gamma_{\mathfrak{p}}\right\} .
\end{gathered}
$$

By Theorem 4.35, we have $\mathfrak{w}_{\mathfrak{p}} \subset \mathfrak{h}_{\mathfrak{p}}$.

From Lemma 4.12 and $\Gamma_{\mathfrak{k}} \subset \Delta_{\mathfrak{k}}$ it follows

$$
\mathfrak{o}_{\mathfrak{p}}=\mathfrak{h}_{\mathfrak{p}} \cap \bigcap_{\gamma \in \Gamma_{\mathfrak{p}}} \mathfrak{k} \mathfrak{e r}(\gamma)=\left\{X \in \mathfrak{h}_{\mathfrak{p}} \mid \forall \gamma \in \Gamma_{\mathfrak{p}} \quad \gamma(X)=0\right\}
$$

Furthermore, by Theorem 4.35 for $\gamma \in \Gamma_{\mathfrak{p}}$ we have that $J E_{\gamma} \in \mathfrak{h}$. As in [6], we shall denote for $\gamma \in \Gamma_{\mathfrak{p}}$

$$
\begin{gathered}
V_{\gamma}=J E_{\gamma} \in \mathfrak{h}_{\mathfrak{p}}^{-}, \quad U_{\gamma}=J E_{-\gamma}=-\tau\left(J E_{\gamma}\right)=-\tau\left(V_{\gamma}\right) \in \mathfrak{h}_{\mathfrak{p}}^{+}, \\
P_{\gamma}=W_{\gamma}-\mathrm{i} I W_{\gamma}, \quad Q_{\gamma}=W_{\gamma}+\mathrm{i} I W_{\gamma} .
\end{gathered}
$$

Proposition 4.37. The complex structure $J$ satisfies the following conditions:

$$
\begin{gathered}
\left|\gamma\left(V_{\gamma}\right)\right|=\left|\gamma\left(U_{\gamma}\right)\right|=1 \quad \gamma \in \Gamma_{\mathfrak{p}} \\
J E_{\gamma}=-\mathrm{i} \gamma\left(V_{\gamma}\right) Q_{\gamma}=\frac{1}{2} \gamma\left(V_{\gamma}\right)\left(H_{\gamma}+\mathrm{i} I H_{\gamma}\right) \quad \gamma \in \Gamma_{\mathfrak{p}} \\
J E_{\alpha}=N_{\gamma,-\alpha} \gamma\left(V_{\gamma}\right) E_{\alpha-\gamma} \quad \alpha \in \Phi_{\gamma}^{+}, \gamma \in \Gamma_{\mathfrak{p}} .
\end{gathered}
$$

The complex structure I satisfies $I\left(\mathfrak{w}_{\mathfrak{p}}\right) \subset \mathfrak{o}_{\mathfrak{p}}$.

Remark 4.38. If we denote $\rho_{\gamma}=\mathrm{i} \overline{\left(V_{\gamma}\right)}$, then the formulas in this proposition are the same as the formulas in the second and the rhird row of (49). We show further that the first formula in (49) is also necessarily valid. 
Proof. By Theorem 4.35 for $\gamma \in \Gamma_{\mathfrak{p}}$ we have $J\left(E_{\gamma}\right)=V_{\gamma} \in \mathfrak{h}, J\left(E_{-\gamma}\right)=U_{\gamma} \in \mathfrak{h}, W_{\gamma} \in \mathfrak{p}_{u}$. Now 10 by the same computations as in the proof of Proposition 4.7 [6] we obtain (146), (147) and

$$
\gamma\left(I H_{\gamma}\right)=0 \quad \gamma \in \Gamma_{\mathfrak{p}} .
$$

If $\gamma \neq \delta, \delta \in \Gamma_{\mathfrak{p}}$, then we compute (117) with $\widetilde{J}=J, \widetilde{\mathfrak{p}}=\mathfrak{p}, X=E_{\gamma}, Y=E_{\delta}$ :

$$
0=\left[J E_{\gamma}, E_{\delta}\right]_{\mathfrak{p}}+\left[E_{\gamma}, J E_{\delta}\right]_{\mathfrak{p}}=\delta\left(V_{\gamma}\right) E_{\delta}-\gamma\left(V_{\delta}\right) E_{\gamma} .
$$

Hence $\delta\left(V_{\gamma}\right)=0$. By (147) $\delta\left(V_{\gamma}\right)=\frac{1}{2} \gamma\left(V_{\gamma}\right) \delta\left(H_{\gamma}+\mathrm{i} I H_{\gamma}\right)=\frac{\mathrm{i}}{2} \gamma\left(V_{\gamma}\right) \delta\left(I H_{\gamma}\right)$. Therefore $\delta\left(I H_{\gamma}\right)=0$ and we see that

$$
\forall \delta \in \Gamma_{\mathfrak{p}} \quad \delta\left(I H_{\gamma}\right)=0 .
$$

If $\delta \in \Gamma_{\mathfrak{k}}$ then $h_{\delta} \in \mathfrak{k}, I H_{\gamma} \in \mathfrak{p}$ and therefore $\delta\left(I H_{\gamma}\right)=\left\langle h_{\delta}, I H_{\gamma}\right\rangle=0$ (see (63) also). So far, we see that $I H_{\gamma} \in \mathfrak{o}$, i. e. $I W_{\gamma} \in \mathcal{o}$ and we proved that $I\left(\mathfrak{w}_{\mathfrak{p}}\right) \subset \mathfrak{o}_{\mathfrak{p}_{u}}$.

We pass to the proof of (148) 11 . Let us fix $\alpha \in \Phi_{\gamma}^{+}, \gamma \in \Gamma_{\mathfrak{p}}$ and denote $\beta=\gamma-\alpha$. From (131) in Theorem 4.35 and (115) we have

$$
J E_{\alpha}=a_{\beta, \alpha} E_{-\beta}+H, \quad H \in \mathfrak{h}_{\mathfrak{p}}^{-} .
$$

In the following computation we put in (117) $\widetilde{J}=J, \widetilde{\mathfrak{p}}=\mathfrak{p}, X=E_{\gamma}, Y=E_{\alpha}$ and keep explicit only terms which nave nontrivial projection to $\mathfrak{h}$.

$$
\begin{gathered}
0=\left[V_{\gamma}, a_{\beta, \alpha} E_{-\beta}+H\right]_{\mid \mathfrak{p}}-J\left[E_{\gamma}, a_{\beta, \alpha} E_{-\beta}+H\right]_{\mid \mathfrak{p}}-J\left[V_{\gamma}, E_{\alpha}\right]_{\mid \mathfrak{p}} \\
=\gamma(H) V_{\gamma}-N_{\gamma,-\beta} a_{\beta, \alpha} J E_{\alpha}-\alpha\left(V_{\gamma}\right) J E_{\alpha}+A \\
=\gamma(H) V_{\gamma}-\left(N_{\gamma,-\beta} a_{\beta, \alpha}+\alpha\left(V_{\gamma}\right)\right) H+B
\end{gathered}
$$

where $A, B \in \mathfrak{n}^{-}$. Now we use $a_{\beta, \alpha}=N_{\gamma,-\alpha} \gamma\left(V_{\gamma}\right)$ (see (121) in Corollary 4.31) and $N_{\gamma,-\alpha} N_{\gamma,-\beta}=-1$ (Proposition 2.27 in [6]) to get

$$
\gamma(H) V_{\gamma}+\beta\left(V_{\gamma}\right) H=0
$$

We apply $\gamma$ to this equation and obtain $\gamma(H)(\gamma+\beta)\left(V_{\gamma}\right)=0$. By (147) and $\beta \in \Phi_{\gamma}^{+}$we have

$$
\beta\left(V_{\gamma}\right)=\gamma\left(V_{\gamma}\right)\left(\frac{1}{2}+\text { imaginary number }\right) \neq 0 .
$$

Thus $(\gamma+\beta)\left(V_{\gamma}\right) \neq 0$, whence $\gamma(H)=0$. Now by (151) and $\beta\left(V_{\gamma}\right) \neq 0$ we get $H=0$. The formula (148) follows from (4.10) and $a_{\beta, \alpha}=N_{\gamma,-\alpha} \gamma\left(V_{\gamma}\right)$.

Definition 4.39. As we show in the next two propositions, it is usefully to introduce some components of $\mathfrak{h}_{\mathfrak{p}}$ as follows:

$$
\begin{gathered}
\mathfrak{v}_{\mathfrak{p}}=\left(\mathfrak{w}_{\mathfrak{p}} \oplus I \mathfrak{w}_{\mathfrak{p}}\right)^{\mathbb{C}}, \quad \mathfrak{v}_{\mathfrak{p}}^{+}=\mathfrak{v}_{\mathfrak{p}} \cap \mathfrak{h}_{\mathfrak{p}}^{+}, \quad \mathfrak{v}_{\mathfrak{p}}^{-}=\mathfrak{v}_{\mathfrak{p}} \cap \mathfrak{h}_{\mathfrak{p}}^{-} \\
\mathfrak{j}_{\mathfrak{p}}^{+}=\mathfrak{o}_{\mathfrak{p}} \cap \mathfrak{h}_{\mathfrak{p}}^{+}, \quad \mathfrak{j}_{\mathfrak{p}}^{-}=\mathfrak{o}_{\mathfrak{p}} \cap \mathfrak{h}_{\mathfrak{p}}^{+}, \quad \mathfrak{j}_{\mathfrak{p}}=\mathfrak{j}_{\mathfrak{p}}^{+} \oplus \mathfrak{j}_{\mathfrak{p}}^{-}
\end{gathered}
$$

\footnotetext{
${ }^{10}$ using the integrability condition in (117) for $J, \mathfrak{p}$

${ }^{11}$ it its the same as the proof of proposition 4.18 in 6 ]
} 
Proposition 4.40. We have

$$
\mathfrak{v}_{\mathfrak{p}}^{+}=\operatorname{span}_{\mathbb{C}}\left\{P_{\gamma} \mid \gamma \in \Gamma_{\mathfrak{p}}\right\}, \quad \mathfrak{v}_{\mathfrak{p}}^{-}=\operatorname{span}_{\mathbb{C}}\left\{Q_{\gamma} \mid \gamma \in \Gamma_{\mathfrak{p}}\right\}, \quad \mathfrak{v}_{\mathfrak{p}}=\mathfrak{v}_{\mathfrak{p}}^{+} \oplus \mathfrak{v}_{\mathfrak{p}}^{-} .
$$

Besides

$$
\mathfrak{h}_{\mathfrak{p}}=\mathfrak{v}_{\mathfrak{p}} \oplus \mathfrak{j}_{\mathfrak{p}}, \quad \mathfrak{h}_{\mathfrak{p}}^{+}=\mathfrak{v}_{\mathfrak{p}}^{+} \oplus \mathfrak{j}_{\mathfrak{p}}^{+}, \quad \mathfrak{h}_{\mathfrak{p}}^{-}=\mathfrak{v}_{\mathfrak{p}}^{-} \oplus \mathfrak{j}_{\mathfrak{p}}^{-}
$$

Proof. The important point here is $I\left(\mathfrak{w}_{\mathfrak{p}}\right) \subset \mathfrak{o}_{\mathfrak{p}}$, proved in Proposition 4.37,

The formulas in (152) are easily verified by dimension count (obviously $\operatorname{span}_{\mathbb{C}}\left\{P_{\gamma} \mid \gamma \in\right.$ $\left.\left.\Gamma_{\mathfrak{p}}\right\} \subset \mathfrak{v}_{\mathfrak{p}}^{+}, \operatorname{span}_{\mathbb{C}}\left\{Q_{\gamma} \mid \gamma \in \Gamma_{\mathfrak{p}}\right\} \subset \mathfrak{v}_{\mathfrak{p}}^{-}\right)$.

The condition $I\left(\mathfrak{w}_{\mathfrak{p}}\right) \subset \mathfrak{o}_{\mathfrak{p}}$ implies that for $\gamma, \delta \in \Gamma_{\mathfrak{p}} \gamma\left(P_{\delta}\right)=0$, if $\delta \neq \gamma$ and $\gamma\left(P_{\gamma}\right)=$ i. On the other hand by (143) $\mathfrak{j}_{\mathfrak{p}}^{+}=\left\{H \in \mathfrak{h}_{\mathfrak{p}}^{+} \mid \forall \gamma \in \Gamma_{\mathfrak{p}} \gamma(H)=0\right\}$, thus $\mathfrak{h}_{\mathfrak{p}}^{+}=\mathfrak{v}_{\mathfrak{p}}^{+} \oplus \mathfrak{j}_{\mathfrak{p}}^{+}$. In the same way $\mathfrak{h}_{\mathfrak{p}}^{-}=\mathfrak{v}_{\mathfrak{p}}^{-} \oplus \mathfrak{j}_{\mathfrak{p}}^{-}$.

Now, in order to prove $\mathfrak{h}_{\mathfrak{p}}=\mathfrak{v}_{\mathfrak{p}} \oplus \mathfrak{j}_{\mathfrak{p}}$, we have to show only that $\mathfrak{v}_{\mathfrak{p}} \cap \mathfrak{j}_{\mathfrak{p}}=\{0\}$. Let $X \in \mathfrak{v}_{\mathfrak{p}} \cap \mathfrak{j}_{\mathfrak{p}}$. We may decompose $X=X^{+}+X^{-}$, where $X^{+} \in \mathfrak{v}_{\mathfrak{p}}^{+}, X^{-} \in \mathfrak{v}_{\mathfrak{p}}^{-}$. Obviously $I\left(\mathfrak{j}_{\mathfrak{p}}\right)=\mathfrak{j}_{\mathfrak{p}}$, hence $I(X)=\mathrm{i} X^{+}-\mathrm{i} X^{-} \in \mathfrak{j}_{\mathfrak{p}}$. The inclusions $X^{+}+X^{-} \in \mathfrak{j}_{\mathfrak{p}}, \mathrm{i} X^{+}-\mathrm{i} X^{-} \in \mathfrak{j}_{\mathfrak{p}}$ imply $X^{+} \in \mathfrak{j}_{\mathfrak{p}}$, $X^{-} \in \mathfrak{j}_{\mathfrak{p}}$, therefore $X^{+} \in \mathfrak{j}_{\mathfrak{p}}^{+} \cap \mathfrak{v}_{\mathfrak{p}}^{+}, X^{-} \in \mathfrak{j}_{\mathfrak{p}}^{-} \cap \mathfrak{v}_{\mathfrak{p}}^{-}$. Now from $\mathfrak{v}_{\mathfrak{p}}^{+} \cap \mathfrak{j}_{\mathfrak{p}}^{+}=\mathfrak{v}_{\mathfrak{p}}^{-} \cap \mathfrak{j}_{\mathfrak{p}}^{-}=\{0\}$ we obtain $X^{+}=X^{-}=0$.

Using Propositions 4.37, 4.40 and repeating the proof of Proposition 4.31 in [6] we obtain:

Proposition 4.41. The complex structure J satisfies:

$$
J\left(\mathfrak{j}_{\mathfrak{p}}^{+}\right)=\mathfrak{j}_{\mathfrak{p}}^{-}
$$

In particular the subspace $\mathfrak{j}_{\mathfrak{p}}$ is $J$-invariant.

Corollary 4.42. The following inequality is valid:

$$
\operatorname{rank}(\mathfrak{g})+\operatorname{srank}(\mathfrak{k}) \geq \operatorname{rank}(\mathfrak{k})+\operatorname{srank}(\mathfrak{g}) \geq \operatorname{rank}\left(\mathfrak{k}_{s}\right)+\operatorname{srank}(\mathfrak{g}) .
$$

Proof. From Theorem 4.35 and Proposition 4.37 we know that for all $\gamma \in \Gamma_{\mathfrak{p}} \quad I\left(W_{\gamma}\right) \in$ $\mathfrak{h}_{\mathfrak{p}_{u}} \cap \mathfrak{o}$ and $W_{\gamma} \in \mathfrak{h}_{\mathfrak{p}_{u}}$. On the other hand $\mathfrak{o} \cap \operatorname{span}_{\mathbb{C}}\left\{W_{\gamma} \mid \gamma \in \Gamma_{\mathfrak{p}}\right\}=\{0\}$. Therefore $\operatorname{dim}_{\mathbb{R}}\left(\mathfrak{h}_{\mathfrak{p}_{u}}\right) \geq 2 \#\left(\Gamma_{\mathfrak{p}}\right)$. Hence $\operatorname{dim}_{\mathbb{R}}\left(\mathfrak{h}_{u}\right)-\operatorname{dim}_{\mathbb{R}}\left(\mathfrak{h}_{\mathfrak{k}_{u}}\right) \geq 2\left(\#(\Gamma)-\#\left(\Gamma_{\mathfrak{k}}\right)\right)$, i. e. $\operatorname{dim}_{\mathbb{R}}\left(\mathfrak{h}_{u}\right)+$ $2 \#\left(\Gamma_{\mathfrak{k}}\right) \geq \operatorname{dim}_{\mathbb{R}}\left(\mathfrak{h}_{\mathfrak{k}_{u}}\right)+2 \#(\Gamma)$. By Corollary 3.3 it follows that $2 \#\left(\Gamma_{\mathfrak{k}}\right)=\operatorname{srank}(\mathfrak{k})$, hence $\operatorname{rank}(\mathfrak{g})+\operatorname{srank}(\mathfrak{k}) \geq \operatorname{rank}(\mathfrak{k})+\operatorname{srank}(\mathfrak{g})$.

4.11. The main theorem. We may now state our main theorem.

Theorem 4.43. Let $\mathbf{U}$ be a compact, connected Lie group and let $\mathbf{K}_{u} \subset \mathbf{U}$ be a closed subgroup such that $\mathbf{U}$ acts effectively on $M=\mathbf{U} / \mathbf{K}_{u}$. Let $\left(I_{M}, J_{M}\right)$ be a LIHCS on $M$. Let $\mathfrak{u}$ and $\mathfrak{k}_{u}$ be the Lie algebras of $\mathbf{U}$ and $\mathbf{K}_{u}$, respectively.

Then $\left(\mathfrak{u}, \mathfrak{k}_{u}\right)$ is a hypercomplex pair and $\left(M, I_{M}, J_{M}\right)$ is associated with it (see Definition 3.15). 


\section{SEMisimple HYPERCOMPLEX PAIRS}

In this section we determine explicitly all hypercomplex pairs $\left(\mathfrak{u}, \mathfrak{k}_{u}\right)$, such that $\mathfrak{u}$ is semisimple. We call these special pairs semisimple hypercomplex pairs and show that they correspond to the simply-connected, compact, hypercomplex spaces. Now we give a precise definition, excluding some trivial situations. The auxiliary notion " weak semisimple hypercomplex pair" is used only in this section.

Definition 5.1. Let $(\mathfrak{a}, \mathfrak{b})$ be a hypercomplex pair. If $\mathfrak{a}$ is semisimple with decomposition into simple ideals $\mathfrak{a}=\mathfrak{a}_{1} \oplus \mathfrak{a}_{2} \oplus \cdots \oplus \mathfrak{a}_{p}$, such that for any $i=1, \ldots, k \mathfrak{b} \cap \mathfrak{a}_{i}$ is a proper subalgebra of $\mathfrak{a}_{i}$, then we say that $(\mathfrak{a}, \mathfrak{b})$ is a semisimple hypercomplex pair.

Weak semisimple hypercomplex pair we shall call a pair $(\mathfrak{a}, \mathfrak{b})$, which satisfies all the conditions of a semisimple hypercomplex pair, except possibly $\operatorname{dim}(\mathfrak{a})-\operatorname{dim}(\mathfrak{b}) \in 4 \mathbb{Z}$, $\operatorname{rank}(\mathfrak{g})+\operatorname{srank}(\mathfrak{k}) \geq \operatorname{rank}(\mathfrak{k})+\operatorname{srank}(\mathfrak{g})$ (see definition 3.5).

The aim of this section is to show that if $(\mathfrak{a}, \mathfrak{b})$ is a semisimple hypercomplex pair, then the simple components of both $\mathfrak{a}$ and $\mathfrak{b}$ are only su algebras. We shall use the explicit description of the stems of the simple Lie algebras from Section 3.3 of [6].

5.1. If $\mathfrak{g}$ is simple and $\operatorname{srank}(\mathfrak{g})=2 \operatorname{rank}(\mathfrak{g})$. We get in this case if $\mathfrak{g}$ is any of the simple compact Lie algebras: $\mathfrak{s o}(4 q, \mathbb{C}), q \geq 2, \mathfrak{s o}(2 d+1, \mathbb{C}), \mathfrak{s p}(d, \mathbb{C}), d \geq 1, \mathfrak{e}_{8}, \mathfrak{e}_{7}, \mathfrak{f}_{4}, \mathfrak{G}_{2}$.

Proposition 5.2. Let $(\mathfrak{g}, \mathfrak{k})$ be a complexified weak semisimple hypercomplex pair with simple $\mathfrak{g}$, such that $\operatorname{srank}(\mathfrak{g})=2 \operatorname{rank}(\mathfrak{g})$. Then

$$
\operatorname{rank}(\mathfrak{g})+\operatorname{srank}(\mathfrak{k})-\operatorname{rank}\left(\mathfrak{k}_{s}\right)-\operatorname{srank}(\mathfrak{g})<0,
$$

where $\mathfrak{k}_{s}$ denotes the semisimple part of the reductive Lie algebra $\mathfrak{k}$.

Proof. Now $\operatorname{srank}(\mathfrak{g})=2 \operatorname{rank}(\mathfrak{g})$ and therefore

$$
\begin{gathered}
\operatorname{rank}(\mathfrak{g})+\operatorname{srank}(\mathfrak{k})-\operatorname{rank}\left(\mathfrak{k}_{s}\right)-\operatorname{srank}(\mathfrak{g})=-\operatorname{rank}(\mathfrak{g})+\operatorname{srank}(\mathfrak{k})-\operatorname{rank}\left(\mathfrak{k}_{s}\right) \\
\leq-\operatorname{rank}(\mathfrak{g})+\operatorname{rank}\left(\mathfrak{k}_{s}\right)
\end{gathered}
$$

where we used the inequality $\operatorname{srank}(\mathfrak{k}) \leq 2 \operatorname{rank}\left(\mathfrak{k}_{s}\right)$, proved in Corollary 2.44 of [6].

Let $\mathcal{M}$ be as in Lemma 3.3 (now, by the definition, $\mathfrak{k}$ is a $\Gamma_{\mathfrak{k}}$-stemmed subalgebra of $\mathfrak{g}$ for some substem $\left.\Gamma_{\mathfrak{k}}\right)$. Then

$$
\mathfrak{k}=(\mathfrak{h} \cap \mathfrak{k})+\sum_{\alpha \in \Delta_{\mathfrak{k}}} \mathfrak{g}(\alpha) \quad \Delta_{\mathfrak{k}}=\bigcup_{\gamma \in \mathcal{M}} \Theta_{\gamma} \quad \Delta_{\mathfrak{k}}^{+}=\bigcup_{\gamma \in \mathcal{M}} \Theta_{\gamma}^{+}
$$

and a basis of $\Delta_{\mathfrak{k}}$ is $\Pi \cap \Delta_{\mathfrak{k}}$, where $\Pi$ is a basis of $\Delta$. Therefore $-\operatorname{rank}(\mathfrak{g})+\operatorname{rank}\left(\mathfrak{k}_{s}\right)=$ $-\#(\Pi)+\#\left(\Pi \cap \Delta_{\mathfrak{k}}\right)$. So we see that $\operatorname{rank}(\mathfrak{g})+\operatorname{srank}(\mathfrak{k})-\operatorname{rank}\left(\mathfrak{k}_{s}\right)-\operatorname{srank}(\mathfrak{g}) \geq 0$ implies $-\#(\Pi)+\#\left(\Pi \cap \Delta_{\mathfrak{k}}\right) \geq 0$, hence $\Pi=\Pi \cap \Delta_{\mathfrak{k}}$. On the other hand $\Pi=\Pi \cap \Delta_{\mathfrak{k}}$ implies $\Delta=\Delta_{\mathfrak{k}}$ and then $\mathfrak{g}=\mathfrak{k}$, which contradicts the definition of weak semisimple hypercomplex pair 5.1 . 
5.2. If $\mathfrak{g}=\mathfrak{s o}(2 p, \mathbb{C}), p=2 q+1, q \geq 2$. Here the root system is $\mathfrak{d}_{p}$. We take $p$ orthonormal vectors $e_{1}, \ldots, e_{p}$ in the standard Euclidean $p$-dimensional space and put

$$
\Delta=\left\{ \pm e_{i} \pm e_{j} \mid i, j=1, \ldots, p, i<j\right\}, \quad \Delta^{+}=\left\{e_{i} \pm e_{j} \mid i, j=1, \ldots, p, i<j\right\} .
$$

Let us recall that the stem is $\Gamma=\left\{\gamma_{1}, \ldots, \gamma_{d}\right\}, d=2 q$, where

$$
\begin{gathered}
\gamma_{1}=e_{1}+e_{2}, \gamma_{2}=e_{3}+e_{4}, \ldots, \gamma_{k}=e_{2 k-1}+e_{2 k}, \ldots, \gamma_{q}=e_{2 q-1}+e_{2 q} \\
\gamma_{q+1}=e_{1}-e_{2}, \ldots, \gamma_{q+k}=e_{2 k-1}-e_{2 k}, \ldots, \gamma_{2 q}=e_{2 q-1}-e_{2 q}
\end{gathered}
$$

In this case the root systems $\Theta_{\gamma}$ for $\gamma \in \Gamma$ are as follows

$$
\begin{gathered}
\Theta_{\gamma_{k}}=\Theta_{e_{2 k-1}+e_{2 k}}=\left\{ \pm e_{i} \pm e_{j} \mid 2 k-1 \leq i<j \leq p\right\}=\mathfrak{d}_{p-2 k+2} \\
\Theta_{\gamma_{q+k}}=\Theta_{e_{2 k-1}+e_{2 k}}=\left\{e_{2 k-1}-e_{2 k}, e_{2 k}-e_{2 k-1}\right\}=\mathfrak{a}_{1} \\
k=1, \ldots, q
\end{gathered}
$$

Therefore

$$
\operatorname{rank}(\mathfrak{g})=p \quad \operatorname{srank}(\mathfrak{g})=2(p-1) .
$$

Now we may depict the partially ordered set $(\Gamma, \prec)$ in the following Hasse diagram

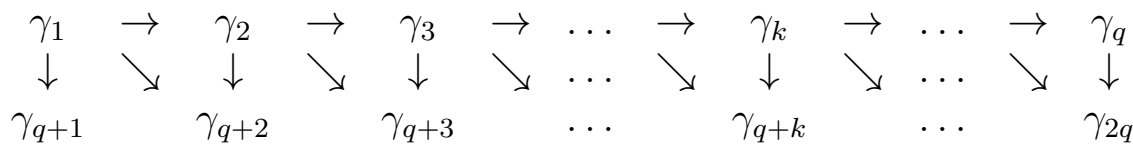

And we see that the subsets of $\Gamma$, which consist of pairwise incomparable elements and do not contain the highest root $\gamma_{1}$, are of the type:

$$
\begin{gathered}
\mathcal{M}=\emptyset \text { or } \\
\mathcal{M}=A \cup\left\{\gamma_{k}\right\} \quad 2 \leq k \leq q, \quad A \subset\left\{\gamma_{q+1}, \gamma_{q+2}, \ldots, \gamma_{q+k-1}\right\} \\
\mathcal{M}=A \quad \text { or } \quad A \subset\left\{\gamma_{q+1}, \gamma_{q+2}, \ldots, \gamma_{2 q}\right\} .
\end{gathered}
$$

Now we shall prove

Proposition 5.3. Let $(\mathfrak{g}, \mathfrak{k})$ be a complexified weak semisimple hypercomplex pair. Let $\mathfrak{g}=$ $\mathfrak{s o}(2 p, \mathbb{C})$, where $p=2 q+1, q \geq 2$. Then $\operatorname{rank}(\mathfrak{g})+\operatorname{srank}(\mathfrak{k})-\operatorname{rank}\left(\mathfrak{k}_{s}\right)-\operatorname{srank}(\mathfrak{g})<0$.

Proof. Let $\mathcal{M}$ be the subset of the stem, which corresponds to $\mathfrak{k}$ as in Lemma 3.3 (see (155) also). Then about $\mathcal{M}$ we have only three possible cases (159), (160), (161).

If $\mathcal{M}=\emptyset$. Then $\operatorname{rank}\left(\mathfrak{k}_{s}\right)=0$ and $\operatorname{srank}(\mathfrak{k})=0$, hence (we recall that $q \geq 2$ )

$$
\operatorname{rank}(\mathfrak{g})+\operatorname{srank}(\mathfrak{k})-\operatorname{rank}\left(\mathfrak{k}_{s}\right)-\operatorname{srank}(\mathfrak{g})=p-2(p-1)=-2 q+1<0 .
$$


If $\mathcal{M}=A \cup\left\{\gamma_{k}\right\}$, where $2 \leq k \leq q, A \subset\left\{\gamma_{q+1}, \gamma_{q+2}, \ldots, \gamma_{q+k-1}\right\}$. In this case $\Delta_{\mathfrak{k}}=\Theta_{\gamma_{k}} \cup \bigcup_{\gamma \in A} \Theta_{\gamma}$, hence by (156) we have

$$
\begin{aligned}
\operatorname{srank}(\mathfrak{k}) & =\operatorname{srank}\left(\Theta_{\gamma_{k}}\right)+\sum_{\gamma \in A} \operatorname{srank}\left(\Theta_{\gamma}\right)= \\
& =\operatorname{srank}\left(\mathfrak{d}_{p-2 k+2}\right)+\#(A) \operatorname{srank}\left(\mathfrak{a}_{1}\right)=2(p-2 k+1)+2 \#(A) \\
\operatorname{rank}\left(\mathfrak{k}_{s}\right) & =\operatorname{rank}(\mathfrak{s o}(2(p-2 k+2), \mathbb{C}))+\#(A) \operatorname{rank}(\operatorname{sl}(2, \mathbb{C})) \\
& =p-2 k+2+\#(A) .
\end{aligned}
$$

In this case we compute:

$$
\begin{gathered}
\operatorname{rank}(\mathfrak{g})+\operatorname{srank}(\mathfrak{k})-\operatorname{rank}\left(\mathfrak{k}_{s}\right)-\operatorname{srank}(\mathfrak{g}) \\
=p+2(p-2 k+1)+2 \#(A)-(p-2 k+2+\#(A))-2(p-1) \\
=2+\#(A)-2 k \leq 2+(k-1)-2 k=1-k<0 .
\end{gathered}
$$

If $\mathcal{M}=A$, where $A \subset\left\{\gamma_{q+1}, \gamma_{q+2}, \ldots, \gamma_{2 q}\right\}$. In this case $\Delta_{\mathfrak{k}}=\bigcup_{\gamma \in A} \Theta_{\gamma}$, hence by (156) we have:

$$
\begin{aligned}
\operatorname{srank}(\mathfrak{k}) & =\sum_{\gamma \in A} \operatorname{srank}\left(\Theta_{\gamma}\right)=\#(A) \operatorname{srank}\left(\mathfrak{a}_{1}\right)=2 \#(A) \\
\operatorname{rank}\left(\mathfrak{k}_{s}\right) & =\#(A) \operatorname{rank}(\operatorname{sl}(2, \mathbb{C}))=\#(A) .
\end{aligned}
$$

Hence (we recall that $q \geq 2$ ):

$$
\begin{gathered}
\operatorname{rank}(\mathfrak{g})+\operatorname{srank}(\mathfrak{k})-\operatorname{rank}\left(\mathfrak{k}_{s}\right)-\operatorname{srank}(\mathfrak{g})=p+2 \#(A) \\
-\#(A)-2(p-1) \leq q-p+2=1-q<0 .
\end{gathered}
$$

5.3. If $\mathfrak{g}=\mathfrak{e}_{6}$. Here $\mathfrak{g}=\mathfrak{e}_{6}$ (we denote by the same symbols the root system $\mathfrak{e}_{6}$ and the corresponding complex simle Lie algebra).

Let us recall that the stem in this case contains four elements and it is linearly ordered, i. e. we may order the elements of $\Gamma$ in a sequence $\Gamma=\left\{\gamma_{1}, \gamma_{2}, \gamma_{3}, \gamma_{4}\right\}$, so that the corresponding Hasse diagram is

$$
\gamma_{1} \rightarrow \gamma_{2} \rightarrow \gamma_{3} \rightarrow \gamma_{4}
$$

These statements follow from the results in Example 3.25, Case 3, [6]. We see that now

$$
\operatorname{rank}(\mathfrak{g})=6, \quad \operatorname{srank}(\mathfrak{g})=8 .
$$

Furthermore, we have shown in Example 3.25, Case 3, [6] that the root systems $\Theta_{\gamma}$ for $\gamma \in \Gamma$ are as follows

$$
\Theta_{\gamma_{1}}=\mathfrak{e}_{6}, \quad \Theta_{\gamma_{2}}=\mathfrak{a}_{5}, \quad \Theta_{\gamma_{3}}=\mathfrak{a}_{3}, \quad \Theta_{\gamma_{4}}=\mathfrak{a}_{1}
$$


And we see that the subsets of $\Gamma$, which consist of pairwise incomparable elements are of the type

$$
\mathcal{M}=\left\{\gamma_{i}\right\} \quad i=1,2,3,4 \text { or } \mathcal{M}=\emptyset .
$$

Proposition 5.4. Let $(\mathfrak{g}, \mathfrak{k})$ be a complexified weak semisimple hypercomplex pair and $\mathfrak{g}=\mathfrak{e}_{6}$. Then $\operatorname{rank}(\mathfrak{g})+\operatorname{srank}(\mathfrak{k})-\operatorname{rank}\left(\mathfrak{k}_{s}\right)-\operatorname{srank}(\mathfrak{g})<0$.

Proof. Let $\mathcal{M}$ be the subset of the stem, which corresponds to $\mathfrak{k}$ as in Lemma 3.3)( see (155) also). Then about $\mathcal{M}$ we have the possibilities (164).

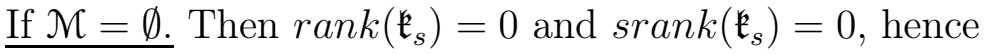

$$
\operatorname{rank}(\mathfrak{g})+\operatorname{srank}(\mathfrak{k})-\operatorname{rank}\left(\mathfrak{k}_{s}\right)-\operatorname{srank}(\mathfrak{g})=6-8=-2<0 .
$$

If $\left.\mathcal{M}=\left\{\gamma_{k}\right\}\right)$, where $k=2,3,4$. In this case $\Delta_{\mathfrak{k}}=\Theta_{\gamma_{k}}$, where $k=2,3,4$, hence by (163) we have (we do not consider $\mathcal{M}=\left\{\gamma_{1}\right\}$, since $\mathfrak{k}$ is a proper subalgebra of $\mathfrak{g}$ ):

$$
\begin{aligned}
& \operatorname{srank}(\mathfrak{k})=\operatorname{srank}\left(\mathfrak{a}_{9-2 k}\right)=\operatorname{srank}\left(\mathfrak{a}_{2(5-k)-1}\right)=2(5-k) \\
& \operatorname{rank}\left(\mathfrak{k}_{s}\right)=\operatorname{rank}(\operatorname{sl}(9-2 k+1, \mathbb{C}))=9-2 k .
\end{aligned}
$$

Using these formlas we compute:

$$
\operatorname{rank}(\mathfrak{g})+\operatorname{srank}(\mathfrak{k})-\operatorname{rank}\left(\mathfrak{k}_{s}\right)-\operatorname{srank}(\mathfrak{g})=6+2(5-k)-(9-2 k)-8=-1<0 .
$$

5.4. If $\mathfrak{g}=\operatorname{sl}(n+1, \mathbb{C}), n \geq 1$. Here $\mathfrak{g}=\operatorname{sl}(n+1, \mathbb{C})$, i. e. the traceless $(n+1) \times(n+1)$ complex matrices. We shall denote by $E_{j}^{i}$ the $(n+1) \times(n+1)$ matrix with 1 at the intersection of the $i$-th row and the $j$-th column and zeros at the others positions, where $i$ and $j$ vary from 1 to $n+1$. Let $\left\{e_{i}\right\}_{i=1}^{n+1}$ be the dual basis of the basis $\left\{E_{i}^{i}\right\}_{i=1}^{n+1}$ of the subspace in $g l(n+1, \mathbb{C})$ of all diagonal matrices. We shall work with the following Cartan subalgebra of $\operatorname{sl}(n+1, \mathbb{C})$

$$
\mathfrak{h}=\operatorname{span}\left\{E_{i}^{i}-E_{i+1}^{i+1} \mid i=1, \ldots, n\right\}
$$

and the following root system and subset of positive roots

$$
\Delta=\left\{e_{i}-e_{j} \mid 1 \leq i \neq j \leq n+1\right\} \quad \Delta^{+}=\left\{e_{i}-e_{j} \mid 1 \leq i<j \leq n+1\right\} .
$$

Let us recall that the stem $\Gamma$ contains $d=\left[\frac{n+1}{2}\right]$ elements and in this case it is linearly ordered, i. e. we may order the elements of $\Gamma$ in a sequence $\Gamma=\left\{\gamma_{1}, \gamma_{2}, \ldots, \gamma_{d}\right\}$, so that the corresponding Hasse diagram is

$$
\gamma_{1} \rightarrow \gamma_{2} \rightarrow \cdots \rightarrow \gamma_{d}
$$

We see that now

$$
\operatorname{rank}(\mathfrak{g})=n, \quad \operatorname{srank}(\mathfrak{g})=2 d=2\left[\frac{n+1}{2}\right] .
$$

Furthermore, we have shown that the root systems $\Theta_{\gamma}$ for $\gamma \in \Gamma$ are as follows

$$
\Theta_{\gamma_{1}}=\mathfrak{a}_{n}, \quad \Theta_{\gamma_{2}}=\mathfrak{a}_{n-2}, \ldots, \Theta_{\gamma_{k}}=\mathfrak{a}_{n-2 k+2}, \ldots, \Theta_{\gamma_{d}}=\mathfrak{a}_{n-2 d+2} .
$$


And now the possibilities for $\mathcal{M}$ are

$$
\mathcal{M}=\left\{\gamma_{i}\right\} \quad i=1, \ldots, d \quad \text { or } \quad \mathcal{M}=\emptyset .
$$

Proposition 5.5. Let $(\mathfrak{g}, \mathfrak{k})$ be a complexified weak semisimple hypercomplex pair and $\mathfrak{g}=$ $\operatorname{sl}(n+1, \mathbb{C}), n \geq 1$. Then if $n$ is odd and $\operatorname{srank}(\mathfrak{k})=0$ we have $\operatorname{rank}(\mathfrak{g})+\operatorname{srank}(\mathfrak{k})-$ $\operatorname{rank}\left(\mathfrak{k}_{s}\right)-\operatorname{srank}(\mathfrak{g})=-1$, otherwise $\operatorname{rank}(\mathfrak{g})+\operatorname{srank}(\mathfrak{k})-\operatorname{rank}\left(\mathfrak{k}_{s}\right)-\operatorname{srank}(\mathfrak{g})=0$.

Proof. If $\mathcal{M}=\emptyset$. Then $\operatorname{rank}\left(\mathfrak{k}_{s}\right)=0$ and $\operatorname{srank}\left(\mathfrak{k}_{s}\right)=0$, hence

$$
\begin{aligned}
\operatorname{rank}(\mathfrak{g})+\operatorname{srank}(\mathfrak{k}) & -\operatorname{rank}\left(\mathfrak{k}_{s}\right)-\operatorname{srank}(\mathfrak{g})=n-2\left[\frac{n+1}{2}\right] \\
= & \left\{\begin{array}{cc}
0 & \text { if } n \text { is even } \\
-1 & \text { if } n \text { is odd }
\end{array} .\right.
\end{aligned}
$$

If $\mathcal{M}=\left\{\gamma_{k}\right\}$, where $k=2, \ldots, d$. In this case $\Delta_{\mathfrak{k}}=\Theta_{\gamma_{k}}$, where $k=2, \ldots, d$, hence by (166) we have (we do not consider $\mathcal{M}=\left\{\gamma_{1}\right\}$, since $\mathfrak{k}$ is a proper subalgebra of $\mathfrak{g}$ ):

$$
\begin{aligned}
\operatorname{srank}(\mathfrak{k}) & =\operatorname{srank}\left(\mathfrak{a}_{n-2 k+2}\right)=2\left[\frac{n-2 k+3}{2}\right]=2\left[\frac{n+1}{2}\right]+2-2 k \\
\operatorname{rank}\left(\mathfrak{k}_{s}\right) & =\operatorname{rank}(\operatorname{sl}(n-2 k+3, \mathbb{C}))=n-2 k+2 .
\end{aligned}
$$

Using these formulas we compute:

$$
\begin{gathered}
\operatorname{rank}(\mathfrak{g})+\operatorname{srank}(\mathfrak{k})-\operatorname{rank}\left(\mathfrak{k}_{s}\right)-\operatorname{srank}(\mathfrak{g}) \\
=n+2\left[\frac{n+1}{2}\right]+2-2 k-(n-2 k+2)-2\left[\frac{n+1}{2}\right]=0 .
\end{gathered}
$$

Remark 5.6. Let $(\mathfrak{g}, \mathfrak{k})$ be a complexified weak semisimple hypercomplex pair, s. $t$. $\mathfrak{g}=\operatorname{sl}(n+$ $1, \mathbb{C}), n \geq 1$, and $\mathfrak{k}$ is a semisimple subalgebra of $\mathfrak{g}$. Then $\mathfrak{k}$ is isomorphic to $\operatorname{sl}(n+3-2 i, \mathbb{C})$ for some $i \in\{2, \ldots, d\}$, which is embedded in $\operatorname{sl}(n+1, \mathbb{C})$ in terms of matrices as follows

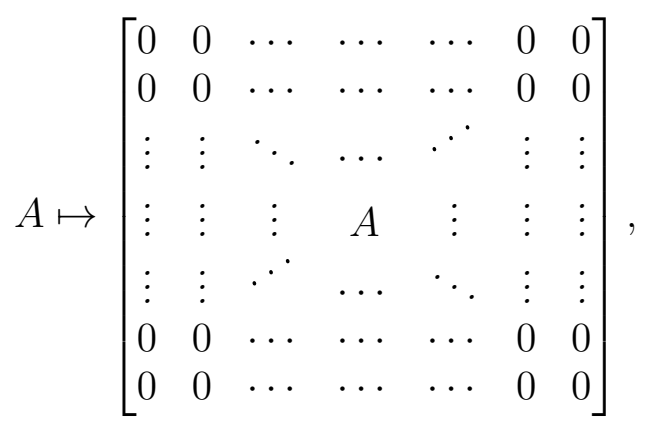

where $A$ is $(n+3-2 i) \times(n+3-2 i)$ matrix and it is nested in a $(n+1) \times(n+1)$ matrix. In this situation the real subalgebra $\mathfrak{k}_{u}=\mathfrak{u} \cap \mathfrak{k}$ is isomorphic to $s u(n+3-2 i)$ and it is embedded in $\mathfrak{u}=s u(n+1)$ in the same way. 


\subsection{Semisimple hypercomplex pairs.}

Definition 5.7. A weak semisimple hypercomplex pair $(\mathfrak{a}, \mathfrak{b})$ will be called su-hypercomplex pair, if $\mathfrak{a} \cong s u(n+1), n \geq 2, \mathfrak{b}$ is of the type $\mathfrak{b} \cong s u(n+3-2 k), k \geq 2, n+3-2 k>0$, and $\mathfrak{b}$ is embedded in $\mathfrak{a} \cong s u(n+1)$ as shown in Remark 5.6. (167).

If $n$ is even we include the pairs with vanishing $\mathfrak{b}, i$. e. we allow in this definition $\mathfrak{b}=\{0\}$, if $\mathfrak{a} \cong s u(n+1)$ with even $n$.

The complexification of an su-hypercomplex pair will be called sl-hypercomplex pair.

From Proposition 5.5 we see that the pairs described here are indeed hypercomplex pairs (not just weak hypercomplex pairs).

Taking into account this definition and Remark 5.6 we see that we may unite Propositions 5.2, 5.3, 5.4, 5.5 in the following proposition 121314 :

Proposition 5.8. Let $\mathfrak{g}$ be a complex simple Lie algebra and $(\mathfrak{g}, \mathfrak{k})$ be a complexified weak semisimple hypercomplex pair. Then $\operatorname{rank}(\mathfrak{g})+\operatorname{srank}(\mathfrak{k})-\operatorname{rank}\left(\mathfrak{k}_{s}\right)-\operatorname{srank}(\mathfrak{g}) \leq 0$.

Equality is attained iff $\left(\mathfrak{g}, \mathfrak{k}_{s}\right)$ is a sl-hypercomplex pair. (We recall that if $\mathfrak{k}$ is abelian, we write $\left.\mathfrak{k}_{s}=\{0\}\right)$.

Now we can prove the final theorem of this section:

Theorem 5.9. Let $\left(\mathfrak{u}, \mathfrak{k}_{u}\right)$ be a semisimple hypercomplex pair with complexification $(\mathfrak{g}, \mathfrak{k})$. Let $\mathfrak{u}=\mathfrak{u}_{1} \oplus \cdots \oplus \mathfrak{u}_{p}$, where $\left\{\mathfrak{u}_{i}\right\}_{i=1}^{p}$ are the simple ideals of $\mathfrak{u}$. Then for $i=1, \ldots, p$ the pair $\left(\mathfrak{u}_{i}, \mathfrak{u}_{i} \cap \mathfrak{k}_{u}\right)$ is an su-hypercomplex pair and $\mathfrak{k}_{u}=\left(\mathfrak{u}_{1} \cap \mathfrak{k}_{u}\right) \oplus\left(\mathfrak{u}_{2} \cap \mathfrak{k}_{u}\right) \cdots \oplus\left(\mathfrak{u}_{p} \cap \mathfrak{k}_{u}\right)$.

In particular every semisimple hypercomplex pair is minimal $i$. e.

$$
\operatorname{rank}(\mathfrak{g})+\operatorname{srank}(\mathfrak{k})-\operatorname{rank}(\mathfrak{k})-\operatorname{srank}(\mathfrak{g})=0 .
$$

Proof. Let $\mathfrak{h}, \mathcal{M}, \Delta, \Delta_{\mathfrak{k}}$ be as in Definition 3.5 and Lemma 3.3 applied for $\left(\mathfrak{u}, \mathfrak{k}_{u}\right)$ and $(\mathfrak{g}, \mathfrak{k})$, i. e.

$$
\begin{gathered}
\mathfrak{k}=\mathfrak{k} \cap \mathfrak{h}+\sum_{\alpha \in \Delta_{\mathfrak{k}}} \mathfrak{g}(\alpha) ; \quad \Delta_{\mathfrak{k}}=\bigcup_{\gamma \in \mathcal{M}} \Theta_{\gamma}, \\
\operatorname{rank}(\mathfrak{g})+\operatorname{srank}(\mathfrak{k}) \geq \operatorname{rank}(\mathfrak{k})+\operatorname{srank}(\mathfrak{g}) \geq \operatorname{rank}\left(\mathfrak{k}_{s}\right)+\operatorname{srank}(\mathfrak{g}),
\end{gathered}
$$

where we denote the semisimple part of $\mathfrak{k}$ by $\mathfrak{k}_{s}$ (if $\mathfrak{k} \subset \mathfrak{h}$, then we write $\mathfrak{k}_{s}=\{0\}$ ).

Let $\mathfrak{g}=\bigoplus_{i=1}^{p} \mathfrak{g}_{i}$ be the decomposition of $\mathfrak{g}$ into complex simple ideals and $\Delta=\cup_{i=1}^{p} \Delta_{i}$ be the decomposition of $\Delta$ into irreducible root systems with positive roots $\left\{\Delta_{i}^{+}\right\}_{i=1}^{p}$. Obviously

\footnotetext{
12 In subsection 5.1, where the algebras $\mathfrak{s o}(4 q)$ are considered, we start from $q=2$. The reason is that $\mathfrak{s o}(4) \cong \mathfrak{s u}(2) \oplus \mathfrak{s u}(2)$.

${ }^{13}$ For dealing with the cases $q=1$ in Proposition 5.3 we recall that $\mathfrak{s o}(6) \cong \mathfrak{s u}(4)$.

${ }^{14}$ From Proposition 5.5 we see that if $(\mathfrak{g}, \mathfrak{k})$ is a complexified weak semisimple hypercomplex pair with $\mathfrak{g}=\operatorname{sl}(1+1)$ (hence by definition $\mathfrak{k}$ is a proper subalgebra of $\mathfrak{g}$, which impose in this case $\operatorname{srank}(\mathfrak{k})=0)$, then $\operatorname{rank}(\mathfrak{g})+\operatorname{srank}(\mathfrak{k})-\operatorname{rank}\left(\mathfrak{k}_{s}\right)-\operatorname{srank}(\mathfrak{g})<0$. That's why in the definition of $\mathfrak{s u}$, resp. $\mathfrak{s l}$, hypercomplex pairs 5.7 we start from $n=2$.
} 
$\mathfrak{g}_{i}$ is the complexification of $\mathfrak{u}_{i}$. Besides

$$
\operatorname{rank}(\mathfrak{g})=\sum_{i=1}^{p} \operatorname{rank}\left(\mathfrak{g}_{i}\right) \quad \operatorname{srank}(\mathfrak{g})=\sum_{i=1}^{p} \operatorname{srank}\left(\mathfrak{g}_{i}\right) .
$$

Let for $i=1, \ldots, p$

$$
\mathcal{M}_{i}=\mathcal{M} \cap \Delta_{i} \quad \Delta_{\mathfrak{k}_{i}}=\bigcup_{\gamma \in \mathcal{M}_{i}} \Theta_{\gamma}=\Delta_{\mathfrak{k}_{i}} \cap \Delta_{\mathfrak{k}}
$$

and let $\mathfrak{k}_{i} \subset \mathfrak{g}_{i}$ be the minimal subalgebra of $\mathfrak{g}$, containing $\sum_{\alpha \in \Delta_{\mathfrak{k}_{i}}} \mathfrak{g}(\alpha)$. Then obviously $\mathfrak{k}_{i}$ is semisimple, if it is not trivial, and $\left(\mathfrak{g}_{i}, \mathfrak{k}_{i}\right)$ is complexified weak semisimple hypercomplex pair. Furthermore, $\mathfrak{k}_{s}$ is the minimal subalgebra of $\mathfrak{g}$, containing $\sum_{\alpha \in \Delta_{\mathfrak{k}}} \mathfrak{g}(\alpha)$ and it is decomposed as follows $\mathfrak{k}_{s}=\oplus_{i=1}^{p} \mathfrak{k}_{i}$, hence

$$
\operatorname{rank}\left(\mathfrak{k}_{s}\right)=\sum_{i=1}^{p} \operatorname{rank}\left(\mathfrak{k}_{i}\right) \quad \operatorname{srank}(\mathfrak{k})=\sum_{i=1}^{p} \operatorname{srank}\left(\mathfrak{k}_{i}\right) .
$$

From (169), (170) and (172) it follows that

$$
\begin{gathered}
\operatorname{rank}(\mathfrak{g})+\operatorname{srank}(\mathfrak{k})-\operatorname{rank}\left(\mathfrak{k}_{s}\right)-\operatorname{srank}(\mathfrak{g}) \\
=\sum_{i=1}^{p} \operatorname{rank}\left(\mathfrak{g}_{i}\right)+\operatorname{srank}\left(\mathfrak{k}_{i}\right)-\operatorname{rank}\left(\mathfrak{k}_{i}\right)-\operatorname{srank}\left(\mathfrak{g}_{i}\right) \geq 0 .
\end{gathered}
$$

Since $\left(\mathfrak{g}_{i}, \mathfrak{k}_{i}\right)$ is complexified weak semisimple hypercomplex pair and $\mathfrak{g}_{i}$ is simple for $i \in$ $\{1, \ldots, p\}$, then from Theorem 5.8 and the inequality above it follows that all the pairs $\left(\mathfrak{g}_{i}, \mathfrak{k}_{i}\right)$ for $i=1, \ldots, p$ are $s l$-hypercomplex pairs and that

$$
\begin{gathered}
\operatorname{rank}(\mathfrak{g})+\operatorname{srank}(\mathfrak{k})-\operatorname{rank}\left(\mathfrak{k}_{s}\right)-\operatorname{srank}(\mathfrak{g}) \\
=\sum_{i=1}^{p} \operatorname{rank}\left(\mathfrak{g}_{i}\right)+\operatorname{srank}\left(\mathfrak{k}_{i}\right)-\operatorname{rank}\left(\mathfrak{k}_{i}\right)-\operatorname{srank}\left(\mathfrak{g}_{i}\right)=0 .
\end{gathered}
$$

If $\mathfrak{k}_{s}$ was a proper subalgebra of $\mathfrak{k}$ then we would obtain $\operatorname{rank}\left(\mathfrak{k}_{s}\right)<\operatorname{rank}(\mathfrak{k})$ and this would give $\operatorname{rank}(\mathfrak{g})+\operatorname{srank}(\mathfrak{k})-\operatorname{rank}(\mathfrak{k})-\operatorname{srank}(\mathfrak{g})<\operatorname{rank}(\mathfrak{g})+\operatorname{srank}(\mathfrak{k})-\operatorname{rank}\left(\mathfrak{k}_{s}\right)-\operatorname{srank}(\mathfrak{g})=0$, which contradicts (169). Therefore $\mathfrak{k}=\mathfrak{k}_{s}$, i. e. $\mathfrak{k}$ is semisimple, if it is non trivial, and $\mathfrak{k}=\oplus_{i=1}^{p} \mathfrak{k}_{i}=\oplus_{i=1}^{p}\left(\mathfrak{k} \cap \mathfrak{g}_{i}\right)$. Recalling that $\mathfrak{g}_{i}$ is the complexification of $\mathfrak{u}_{i}$ and that $\mathfrak{k}$ is the complexification of $\mathfrak{k}_{u}$, we see that $\mathfrak{k}_{u}=\oplus_{i=1}^{p}\left(\mathfrak{k}_{u} \cap \mathfrak{u}_{i}\right)$. Besides we showed that $\left(\mathfrak{g}_{i}, \mathfrak{k}_{i}\right)$ are $s l$-hypercomplex pairs, therefore $\left(\mathfrak{u}_{i}, \mathfrak{k}_{u} \cap \mathfrak{u}_{i}\right)$ are su-hypercomplex pairs. The theorem is proved.

Obviously, Theorem 5.9 shows that the semisimple hypercomplex pairs are exactly the direct sums of su-hypercomplex pairs. 
5.6. HC spaces. Here we shall give a list of all the simply connected, compact, hypercomplex, homogeneous spaces (briefly HC-spaces).

In Theorem 5.9 we characterized the semisimple hypercomplex pairs. Here we shall prove:

Theorem 5.10. Any HC space is associated with a semisimple hypercomplex pair.

So, we are given a $\mathrm{HC}$-space $\left(M, I_{M}, J_{M}\right)$. Let $\mathbf{G}_{I}$ be the group of all biholomorphisms of the complex manifold $\left(M, I_{M}\right)$. Bochner and Montgomery 3] have shown that $\mathbf{G}_{I}$ has a real analytic structure/ such that $\mathbf{G}_{I}$ is a Lie group and the action

$$
\mathbf{G}_{I} \times M \rightarrow M \quad(f, m) \in \mathbf{G}_{I} \times M \mapsto f(m) \in M,
$$

is smooth.

Let us denote $\mathbf{G}=\left\{f \in \mathbf{G}_{I} \mid \mathrm{d} f \circ J_{M}=J_{M} \circ \mathrm{d} f\right\}$. Obviously $\mathbf{G}$ is a subgroup of $\mathbf{G}_{I}$ and it is exactly the group of all hypercomplex diffeomorphisms of $M$. One can easily show that $\mathbf{G}$ is a closed subgroup of $\mathbf{G}_{I}$ and therefore it is a Lie subgroup of $\mathbf{G}_{I}$. Thus, the mapping

$$
\mathbf{G} \times M \rightarrow M \quad(f, m) \mapsto f(m)
$$

is a smooth transitive action of $\mathbf{G}$ on $\mathbf{M}$.

By Montgomery (see [11]) we know that $\mathbf{G}$ contains a compact subgroup, say $\mathbf{U}_{0}$, which acts transitively on $M$. Now from [16, p. 4] we conclude that the maximal semisimple subgroup of $\mathbf{U}_{0}$, which we shall denote by $\mathbf{U}$, acts transitively on $M$. Thus far, we obtained a semisimple, compact, connected Lie group $\mathbf{U}$, which acts transitively by hypercomplex diffeomorphisms on $M$. It acts effectively also, since $\mathbf{U} \subset \mathbf{G}$. Hence we obtain that $M$ is a coset of the type

$$
\mathbf{U} / \mathbf{K}_{u} \simeq M
$$

Since $M$ is simply connected then $\mathbf{K}_{u}$ is a connected subgroup. We denote the Lie algebras of $\mathbf{U}$ and $\mathbf{K}_{u}$ by $\mathfrak{u}$ and $\mathfrak{k}_{u}$, respectively.

Lemma 5.11. Let $\mathfrak{u}=\mathfrak{u}_{1} \oplus \mathfrak{u}_{2} \oplus \cdots \oplus \mathfrak{u}_{p}$ be the decomposition of the compact Lie algebra $\mathfrak{u}$ into simple ideals. Then for any $i=1, \ldots, k$ we may assume that $\mathfrak{k}_{u} \cap \mathfrak{u}_{i}$ is a proper subalgebra of $\mathfrak{u}_{i}$.

Proof. If for some $i$ we have $\mathfrak{k}_{u} \supset \mathfrak{u}_{i}$, for simplicity let us take $i=1$, then let us denote by $\mathbf{U}^{\prime}$ the connected subgroup of $\mathbf{U}$ generated by $\mathfrak{u}_{2} \oplus \cdots \oplus \mathfrak{u}_{p}$. Since $\mathfrak{u}_{2} \oplus \cdots \oplus \mathfrak{u}_{p}$ is a compact semisimple Lie algebra, then $\mathbf{U}^{\prime}$ is a compact subgroup of $\mathbf{U}$. By in : $\mathbf{U}^{\prime} \rightarrow \mathbf{U}$ we shall denote the corresponding embedding. Now we consider the coset space $\mathbf{U}^{\prime} /\left(\mathbf{K}_{u} \cap \mathbf{U}^{\prime}\right)$ and the map

$$
\varphi: \mathbf{U}^{\prime} /\left(\mathbf{K}_{u} \cap \mathbf{U}^{\prime}\right) \rightarrow \mathbf{U} / \mathbf{K}_{u} \quad u\left(\mathbf{K}_{u} \cap \mathbf{U}^{\prime}\right) \mapsto u \mathbf{K}_{u}
$$

Let $\pi^{\prime}: \mathbf{U}^{\prime} \rightarrow \mathbf{U}^{\prime} /\left(\mathbf{K}_{u} \cap \mathbf{U}^{\prime}\right)$ and $\pi: \mathbf{U} \rightarrow \mathbf{U} / \mathbf{K}_{u}$ be the corresponding projections. Obviously $\varphi \circ \pi^{\prime}=\pi \circ i n$ and $\varphi$ is smooth injection. Furthermore, $\varphi$ is regular. Indeed, let $X \in \mathfrak{u}_{2} \oplus \cdots \oplus$ $\mathfrak{u}_{p}$ be such that $\mathrm{d} \varphi\left(\mathrm{d} \pi^{\prime}(X)\right)=0$, hence $\mathrm{d} \pi(\mathrm{d} \operatorname{in}(X))=0$, which implies $X \in \mathfrak{k}_{u} \cap\left(\mathfrak{u}_{2} \oplus \cdots \oplus \mathfrak{u}_{p}\right)$. Since the Lie algebra of $\mathbf{K}_{u} \cap \mathbf{U}^{\prime}$ is $\mathfrak{k}_{u} \cap\left(\mathfrak{u}_{2} \oplus \cdots \oplus \mathfrak{u}_{p}\right)$, then $\mathrm{d} \pi^{\prime}(X)=0$ and the regularity of $\varphi$ is proved. On the other hand, since $\mathfrak{u}_{1} \subset \mathfrak{k}_{u}$, then $\mathfrak{k}_{u}=\mathfrak{u}_{1} \oplus \mathfrak{k}_{u} \cap\left(\mathfrak{u}_{2} \oplus \cdots \oplus \mathfrak{u}_{p}\right)$, i. e. 
$\operatorname{dim}\left(\mathbf{K}_{u} \cap \mathbf{U}^{\prime}\right)=\operatorname{dim}\left(\mathfrak{k}_{u}\right)-\operatorname{dim}\left(\mathfrak{u}_{1}\right)$. Therefore $\operatorname{dim}(\mathbf{U})-\operatorname{dim}\left(\mathbf{K}_{u}\right)=\operatorname{dim}(\mathfrak{u})-\operatorname{dim}\left(\mathfrak{k}_{u}\right)=$ $\operatorname{dim}(\mathfrak{u})-\operatorname{dim}\left(\mathfrak{u}_{1}\right)-\left(\operatorname{dim}\left(\mathfrak{k}_{u}\right)-\operatorname{dim}\left(\mathfrak{u}_{1}\right)\right)=\operatorname{dim}\left(\mathbf{U}^{\prime}\right)-\operatorname{dim}\left(\mathbf{K}_{u} \cap \mathbf{U}^{\prime}\right)$. Thus we see that $\operatorname{dim}\left(\mathbf{U}^{\prime} /\left(\mathbf{K}_{u} \cap \mathbf{U}^{\prime}\right)\right)=\operatorname{dim}\left(\mathbf{U} / \mathbf{K}_{u}\right)$, hence $\varphi$ is an open map, therefore it is surjective and therefore $\mathbf{U}^{\prime}$ acts transitively on $M$ as well as $\mathbf{U}$. Therefore we may substitute $\mathbf{U}^{\prime}$ for $\mathbf{U}$. Now the validity of this lemma is obvious.

By the main Theorem $4.43\left(\mathfrak{u}, \mathfrak{k}_{u}\right)$ is a hypercomplex pair (see Definition 3.5) and $M=$ $\mathbf{U} / \mathbf{K}_{u}$ is associated with it (see Definition [3.15). Furthermore, Lemma 5.11 shows that $\left(\mathfrak{u}, \mathfrak{k}_{u}\right)$ is a semisimple hypercomplex pair and Theorem 5.10 is proved.

Now we can prove:

Corollary 5.12. The HC spaces are exactly the coset spaces $\mathbf{U} / \mathbf{K}_{u}$, with the LIHCS, given in the following list:

$$
\begin{gathered}
S U\left(n_{1}+1\right) / S U\left(n_{1}+3-2 k_{1}\right) \times S U\left(n_{2}+1\right) / S U\left(n_{2}+3-2 k_{2}\right) \times \ldots \\
\cdots \times S U\left(n_{l}+1\right) / S U\left(n_{l}+3-2 k_{l}\right) \times S U\left(m_{1}+1\right) \times S U\left(m_{2}+1\right) \times \cdots \times S U\left(m_{p}+1\right)
\end{gathered}
$$

where:

- $l+p \geq 1, l, p \in \mathbb{N}$

- $\forall i \in\{1, \ldots, l\} \quad n_{i} \geq 2, k_{i} \geq 2, n_{i}+3-2 k_{i}>0$;

- $\forall i \in\{1, \ldots, p\} \quad m_{i} \in 2 \mathbb{N}, m_{i} \geq 2$

- for $i \in\{1, \ldots, l\} S U\left(n_{i}+3-2 k_{1}\right)$ is embedded in $S U\left(n_{i}+1\right)$ as shown in (167).

Proof. From Theorem 5.10 we see that any $H C$-space $\left(M, I_{M}, J_{M}\right)$ is of the type $M=$ $\widetilde{\mathbf{U}} / \widetilde{\mathbf{K}}_{u}$, where the Lie algebras $\mathfrak{u}$ and $\mathfrak{k}$ of $\widetilde{\mathbf{U}}$ and $\widetilde{\mathbf{K}}_{u}$ are such that $(\mathfrak{u}, \mathfrak{k})$ is a semi-simple hypercomplex pair and $\left(I_{M}, J_{M}\right)$ is a LIHCS on $\widetilde{\mathbf{U}} / \widetilde{\mathbf{K}}_{u}$, determined by hypercomplex structure $(I, J)$ on $\mathfrak{p}_{u}$ (see Definition 3.15). Furthermore, the hypercomplex structure $(I, J)$ on $\mathfrak{p}_{u}$ is obtained by the method in subsection 3.2.

From Theorem 5.9 and the definition of su-hypercomplex pair 5.7, we see that there is a coset $\mathbf{U} / \mathbf{K}_{u}$ in our list, s. t. the Lie algebras of $\mathbf{U}, \mathbf{K}_{u}$ are $\mathfrak{u}, \mathfrak{k}$, respectively. The space $\mathbf{U} / \mathbf{K}_{u}$ together with the LIHCS on it obtained by left translation of $(I, J)$ on $\mathfrak{p}_{u}$ throughout $\mathbf{U} / \mathbf{K}_{u}$ give another HC space $\left(\mathbf{U} / \mathbf{K}_{u}, I_{U / K}, J_{U / K}\right)$ (see the end of subsection 3.2)

Since $\mathbf{U}, \mathbf{K}_{u}$ are simply connected, then one can easily show that the identity map $I d$ : $(\mathfrak{u}, \mathfrak{k}) \rightarrow(\mathfrak{u}, \mathfrak{k})$ extends to a hypercomplex smooth map $\left(\mathbf{U} / \mathbf{K}_{u}, I_{U / K}, J_{U / K}\right) \rightarrow\left(\widetilde{\mathbf{U}} / \widetilde{\mathbf{K}}_{u}, I_{M}, J_{M}\right)$, furthermore this is a covering map. Since $\mathbf{U} / \mathbf{K}_{u}, \widetilde{\mathbf{U}} / \widetilde{\mathbf{K}}_{u}$ are both simply-connected, then this map is diffeomorphism.

\section{REFERENCES}

[1] Akhiezer D., Lie Group Actions in Complex Analysis, Aspects of Mathematics, 199520

[2] Bedulli L., Gori A., Podesta F., Homogeneous hypercomplex structures and the Joyce construction, Arxiv Math DG 1004.5238 3

[3] Bochner S. and Montgomery D., Locally Compact Groups of Differentiable Transformations, Annals of Mathematics, 47,(1946), 639-653. 15, 44

\footnotetext{
${ }^{15}$ Any coset of the type $S U(n) / S U(m)$ with $m<n$ is simply connected.
} 
[4] Bochner S. and Montgomery D., Groups of Analityc Manifolds, Annals of Mathematics, 48,(1947), 659-669.15

[5] Bourbaki N., Groupes et algebres de Lie, Ch. VII, VIII, Hermann, Paris, 197522

[6] Dimitrov G., Tsanov V., Homogeneous hypercomp;ex structuresI. The compact Lie groups , Arxiv math.DG/1005.0172. 2, 3, 4, 5, 6, 7, 8, 12, 27, 29, 34, 35, 36, 37, 39,

[7] Helgason S., Differential Geometry, Lie Groups, and Symmetric Spaces, Academic Press, 1978 4, 15, 16

[8] Humphreys J. E., Introduction to Lie algebras and representation theory, Springer Ferlag 1978

[9] Joyce D., Compact hypercomplex and quaternionic manifolds, J. Diff. Geom., 35(1992), 743 - 761

[10] Kobayashi S. and K. Nomizu, Foundations of Differential Geometry, V. II, Interscience Publishers, New York-London-Sydney, 1969 14, 21, 28

[11] Montgomery D.,Simply Conected Homogeneous Spaces, Proceedings of the American Mathematical Society, 1,(1950), 467-469. 44

[12] Morimoto A., Structures complexes invariantes sur les groupes de Lie semisimples, C.R. Acad Sci.,Paris 242(1956) $1101-1103$

[13] Onishchik A.L., Lectures on Real Semisimple Lie Algebras and their Representations, ESI Lectures in Mathematics and Physics European Mathematical Society, 2004.

[14] Snow D., Invariant complex structures on reductive Lie groups, J Reine Angew. Math.,371 (1986), 191 - 215. 6

[15] Tits, J. Espaces homogï£¡nes complexes compacts. Commentarii Mathematici Helvetici, Vol.37 (19621963)

[16] Wang H. C., Closed manifolds with a homogeneous complex structure, Amer. J. Math., 76(1954) 1-32 16. 44

[17] Warner G., Harmonic Analysis on Semisimple Lie Groups I, Springer Verlag 1972

[18] Wolf J.A., Complex homogeneous contact manifolds and quaternionic symmetric spaces, Jour. Math. Mech. (Indiana Univ. Math. J.) 14(6)(1965),1033-1047

[19] Wolf J.A., The action of a real semisimple Lie group on a complex flag manifold. I: Orbit structure and holomorphic arc components, Bull. Amer. Math. Soc. 75(1969),1121-123720

[20] Zhelobenko D.P., Compact Lie groups and their representations, Amer. Math. Soc. (1973) (Translated from Russian)

(Dimitrov) University of Vienna, Faculty of Mathematics, Vienna, Austria, E-mail address: gkid@abv.bg

(Tsanov) University of Sofia "St. Kl. Ohridski", Faculty of Mathematics and Informatics,, Sofia, Bulgaria,

E-mail address: tsanov@fmi.uni-sofia.bg 